\title{
PrEP use among men who have sex with men in the Netherlands
}

Citation for published version (APA):

van Dijk, M. (2021). PrEP use among men who have sex with men in the Netherlands: Interest, attitudes, and behaviors in a context of emerging PrEP availability. [Doctoral Thesis, Maastricht University]. Maastricht University. https://doi.org/10.26481/dis.20210331md

Document status and date:

Published: 01/01/2021

DOI:

10.26481/dis.20210331md

Document Version:

Publisher's PDF, also known as Version of record

\section{Please check the document version of this publication:}

- A submitted manuscript is the version of the article upon submission and before peer-review. There can be important differences between the submitted version and the official published version of record.

People interested in the research are advised to contact the author for the final version of the publication, or visit the DOI to the publisher's website.

- The final author version and the galley proof are versions of the publication after peer review.

- The final published version features the final layout of the paper including the volume, issue and page numbers.

Link to publication

\footnotetext{
General rights rights.

- You may freely distribute the URL identifying the publication in the public portal. please follow below link for the End User Agreement:

www.umlib.nl/taverne-license

Take down policy

If you believe that this document breaches copyright please contact us at:

repository@maastrichtuniversity.nl

providing details and we will investigate your claim.
}

Copyright and moral rights for the publications made accessible in the public portal are retained by the authors and/or other copyright owners and it is a condition of accessing publications that users recognise and abide by the legal requirements associated with these

- Users may download and print one copy of any publication from the public portal for the purpose of private study or research.

- You may not further distribute the material or use it for any profit-making activity or commercial gain

If the publication is distributed under the terms of Article $25 \mathrm{fa}$ of the Dutch Copyright Act, indicated by the "Taverne" license above, 

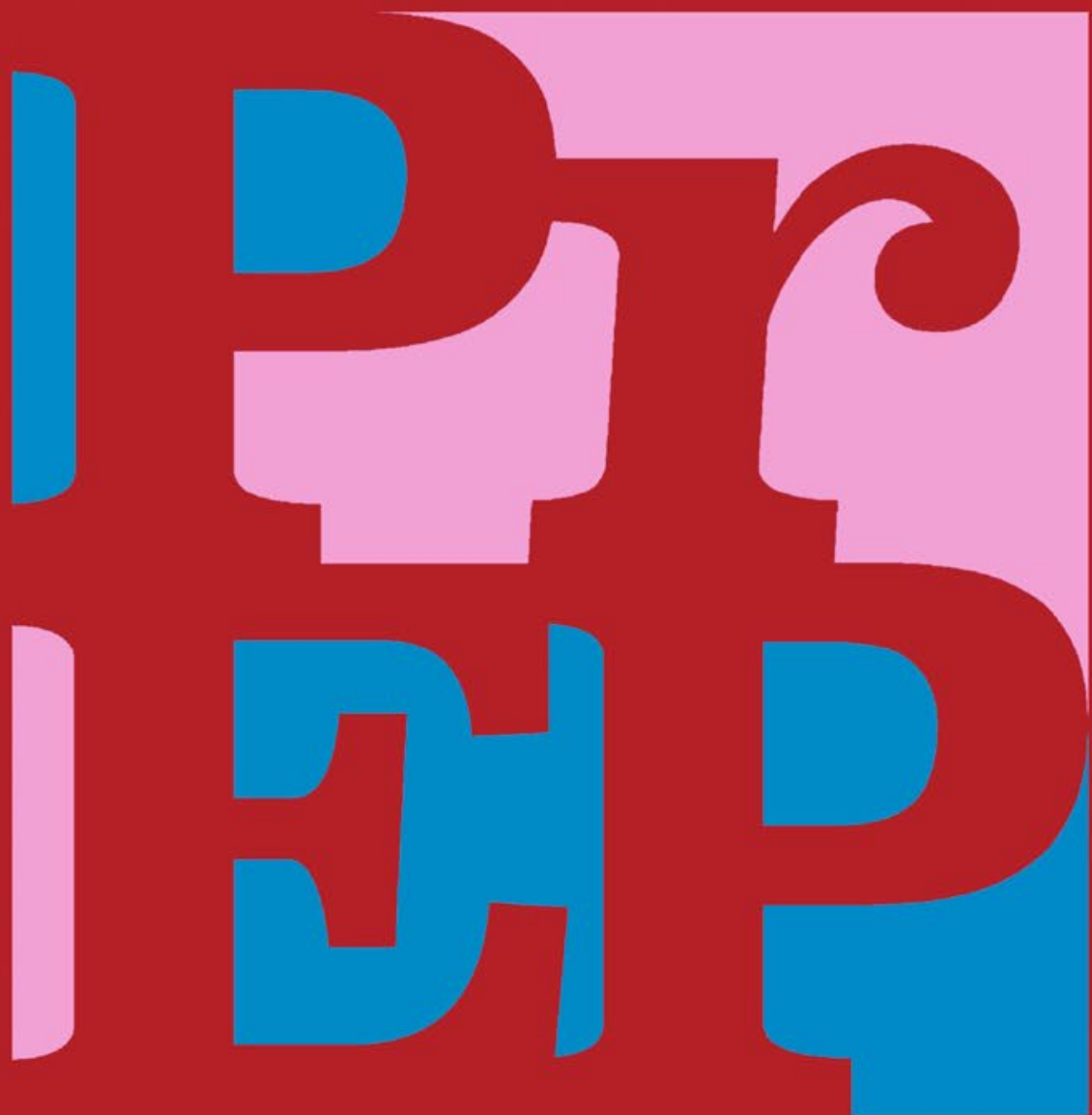

\section{PrEP use among men who have sex with men in the Netherlands}

Interest, attitudes, and behaviors in a context of emerging PrEP availability

Mart van Dijk 

PrEP use among men who have sex with men in the Netherlands:

Interest, attitudes, and behaviors in a context of emerging PrEP availability

Mart van Dijk 
The illustrations on the cover and on the chapter title pages are a tribute to the artwork AIDS by the artist collective General Idea (1987). This artwork made an important contribution to raising awareness and decreasing stigma and fear of HIV/AIDS in the late 1980s and early 1990s. The image was widely distributed, for example as posters, stamps, and wallpaper. By normalising the word 'AIDS' the artists aimed to also normalise the perception of the virus and the disease. The AIDS artwork was inspired by the artwork LOVE by Robert Indiana (1966). LOVE was an icon in the 1960s that symbolised sexual freedom. In my opinion, the letters of PrEP fit into this context because PrEP influences the experience of sexual freedom and PrEP contributes to reducing HIV stigma.

ISBN/EAN: 978-90-831295-6-3

Cover design and illustrations: Darío Rosillo Alarcón

Printed by: Print Service Ede - www.proefschriftenprinten.nl

(C) Mart van Dijk, 2021

The research presented in this dissertation was conducted at the department of Work and Social Psychology, Maastricht University.

This research was funded by AidsFonds (grant number P-13301).

All rights are reserved. No part of this book may be reproduced, distributed, or transmitted in any form or by any means, without prior written permission of the author. 
PrEP use among men who have sex with men in the Netherlands:

Interest, attitudes, and behaviors in a context of emerging PrEP availability

\section{DisSERTATION}

to obtain the degree of Doctor at the Maastricht University, on the authority of the Rector Magnificus Prof. dr. Rianne M. Letschert in accordance with the decision of the Board of Deans, to be defended in public on Wednesday 31 March 2021 at 13:00 hours.

by

Mart van Dijk 


\section{Promotors}

Prof. dr. Kai J. Jonas

Prof. dr. John B.F. de Wit (Utrecht University)

\section{Assessment committee}

Em. Prof. dr. Gerjo Kok (chair)

Prof. dr. Marie Laga (Institute of Tropical Medicine Antwerp)

Prof. dr. Maarten Schim van der Loeff (University of Amsterdam)

Dr. Sarah Stutterheim 


\section{Table of contents}

$\begin{array}{lll}\text { Chapter } 1 \quad \text { General introduction } & 9\end{array}$

Chapter 2 PrEP interest among MSM in the Netherlands: 25

Covariates and differences across samples

Chapter $3 \quad$ Socio-sexual experiences and access to healthcare among 43

informal PrEP users in the Netherlands

Chapter $4 \quad$ Slow uptake of PrEP: Behavioral predictors and the influence of price on PrEP uptake among MSM with a high interest in PrEP

$\begin{array}{lll}\text { Chapter } 5 & \text { Quality of sex life and perceived sexual pleasure of informal } & 81\end{array}$ PrEP users in the Netherlands

Chapter 6 Bridging the serodivide: Attitudes of PrEP users towards sex 95 partners living with HIV

$\begin{array}{lll}\text { Chapter } 7 & \text { General discussion } & 105\end{array}$

$\begin{array}{ll}\text { References } & 119\end{array}$

Summary 151

$\begin{array}{ll}\text { Samenvatting } & 157\end{array}$

Impact paragraph 163

$\begin{array}{ll}\text { Dankwoord } & 167\end{array}$

$\begin{array}{ll}\text { Curriculum vitae } & 171\end{array}$ 


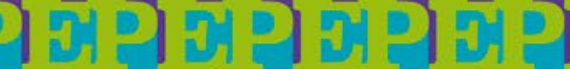

PO

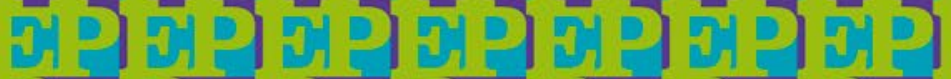

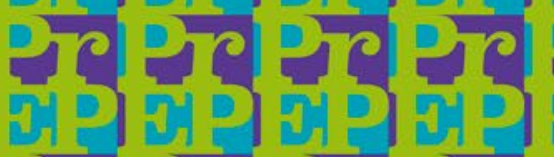

Po

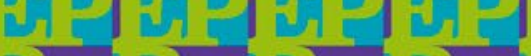

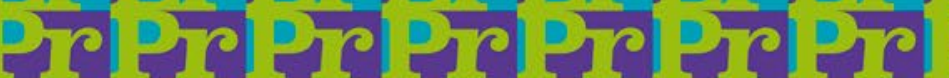

2010

DQ

10

10

객

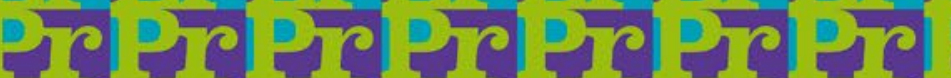

10

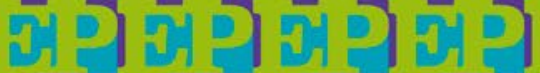

if

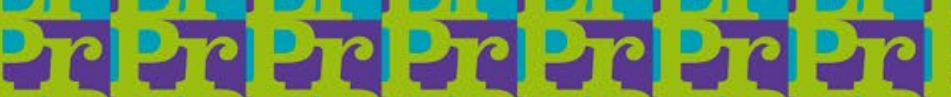

if

7 ,

DO DO DO

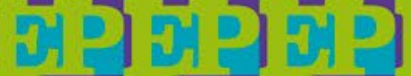

90

1010

To

$50=000$

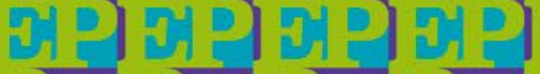

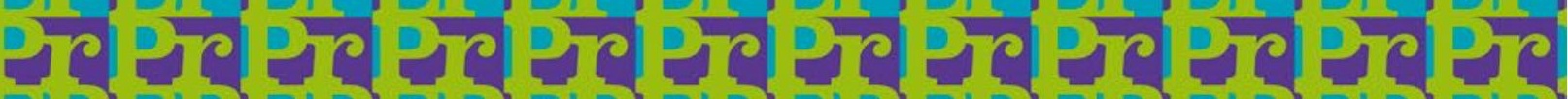

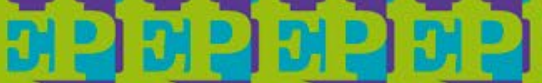

- 000

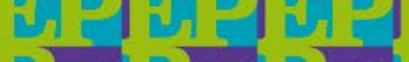

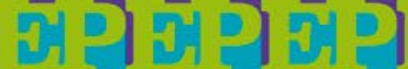

10

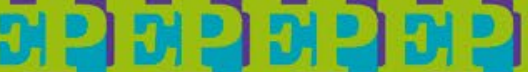

-

10

10

10

10

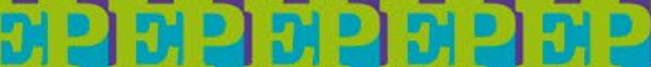

DO DO DO DO DO

200

-

1

-

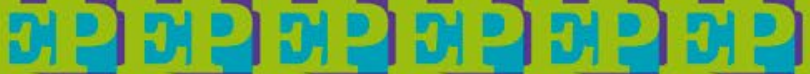

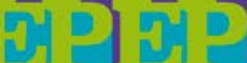

I D

10

DO

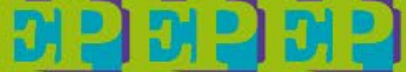

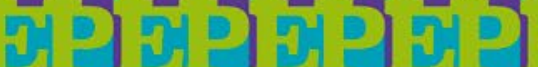

DQ 0

10

100

10

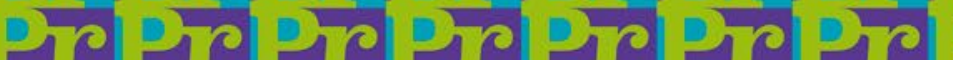

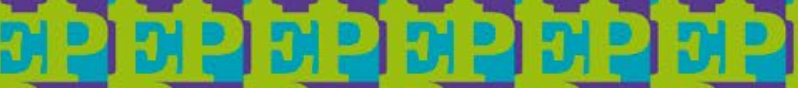

12

it

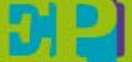

H

10

10

10

10 वO

10

10

매 D

-10 DO DO

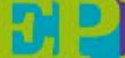

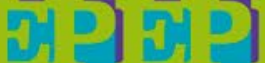

개

걱

$V_{1} D$

D?

$D C$

$\rightarrow 0$

$D C$

100

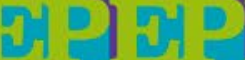

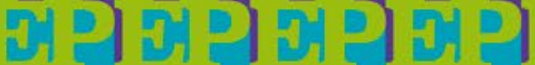

10 DO DO DO

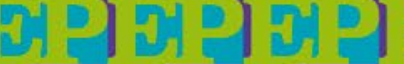

개

in 1

10

प0 जि

90 प0

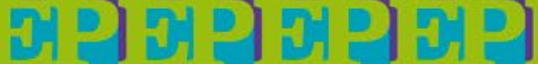

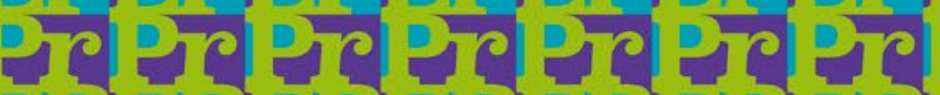

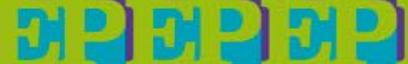

I.

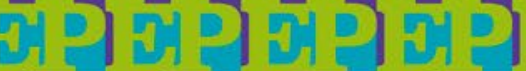

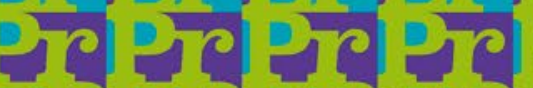

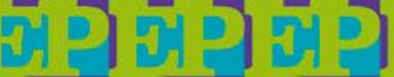

DQ DO DO DO DO

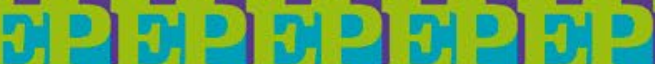

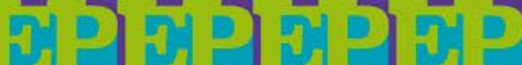

To v0

10

Tre

To

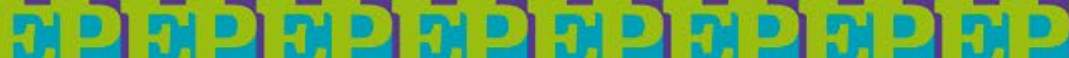




\section{CHAPTER 1 General introduction}


Pre-exposure prophylaxis (PrEP) is a biomedical intervention to prevent HIV infections among HIV-negative individuals. PrEP has been found to be effective in reducing HIV infections among several populations who are at high risk for HIV, such as serodiscordant couples and men who have sex with men (MSM) (Baeten et al., 2012; Grant et al., 2010). Despite the high effectiveness of PrEP, the uptake of PrEP has been limited in the past years, because PrEP has not directly been implemented in all healthcare systems around the world. The European Medicines Agency (EMA) approved PrEP in August 2016 (European Medicines Agency, 2016), and three years later, PrEP became implemented in the healthcare system in the Netherlands (Ministry of Health Welfare and Sport, 2019). Because of the limited accessibility and availability of PrEP during these years, some MSM obtained PrEP via informal channels such as (online) pharmacies abroad, clinics in other countries, and through pill sharing of people living with HIV who take antiretroviral therapy (Charpentier et al., 2014; Kurtz \& Buttram, 2016; Rosenthal et al., 2014; Zablotska et al., 2013). Because informal PrEP users are usually not medically supervised, concerns were raised about misinformation and incorrect use of PrEP (Buttram, 2018; Dimitrov et al., 2013; Kurtz \& Buttram, 2016). The combination of incorrect PrEP use and increased sexual risk-taking may put informal PrEP users at increased risk of HIV (Buttram \& Kurtz, 2017). This thesis aims to assess whether informal PrEP use also occurs in the Netherlands, and what risks are related to informal PrEP use. Therefore, we investigated the interest in PrEP and the experiences of PrEP users in the years prior to formal PrEP implementation in the Netherlands.

This general introduction provides background information on the context of PrEP use in the Netherlands. First, it describes the HIV epidemic, worldwide and in the Netherlands, to emphasize the need for PrEP. Second, it presents the introduction of PrEP. Third, it describes the fragmented implementation of PrEP around the world, and its impact on the availability and use of PrEP. Fourth, it discusses challenges that emerged after the introduction of PrEP, that form the basis of the research questions that this thesis aims to answer. Finally, an outline of the chapters is provided, illustrating how the research aims are related to the studies that were conducted for this thesis.

\section{Epidemiology and prevention of HIV in the age of antiretroviral treatment advances}

It is estimated that 38 million people worldwide are living with HIV (World Health Organization, 2020). In 2019, 1.7 million people got newly infected with HIV, and 700.000 people died due to AIDS. Globally, the yearly number of new infections and deaths have been decreasing since 2000, as a result of the introduction of antiretroviral treatment and developments in 
HIV prevention and education. However, despite these global improvements in HIV incidence and mortality, there are regions where the number of new infections have been increasing in the past decade, such as Eastern Europe and Central Asia (+29\%), Latin America (+7\%), and Middle East and North Africa (+10\%) (UNAIDS, 2019). In a global world, these regional increases make clear that continuous efforts are needed to control the HIV epidemic also in regions with stable or decreasing incidence.

There are 23.300 people living with HIV in the Netherlands (Van Sighem et al., 2019). Since 2008, the number of yearly new infections is decreasing, down to 664 new HIV diagnoses in 2018. The Netherlands reached the UNAIDS 90-90-90 HIV treatment targets (90\% of people living with HIV know their HIV status, of whom $90 \%$ are on antiretroviral treatment and of whom $90 \%$ are virally suppressed) with a continuum of care of 92-93-96 in 2018 (Van Sighem et al., 2019).

MSM have an increased risk for HIV (Beyrer et al., 2012). In the Netherlands, $66 \%$ of HIV infections are among MSM, and in the European Union sex between men is the predominant mode of HIV transmission (European Centre for Disease Prevention and Control, 2019b). It is preferable that multiple HIV prevention strategies are implemented for MSM, as available prevention tools are insufficient as single interventions (Beyrer et al., 2013). Existing effective prevention tools included frequent HIV testing (Wainberg et al., 2016), condom use (Smith, Herbst, et al., 2015) and post-exposure prophylaxis (PEP, the administration of antiretroviral treatment within 72 hours after exposure for 28 days) (Almeda et al., 2004). Recent developments in biomedical HIV prevention methods, such as treatment as prevention (TasP) and PrEP, provide opportunities to further reduce new HIV infections.

\section{Effectiveness and safety of PrEP}

Clinical trials demonstrated that the daily use of the combination of tenofovir and emtricitabine (marketed as Truvada ${ }^{\oplus}$ by Gilead Sciences, and by now also available as a generic formulation in many countries) for PrEP is highly effective in reducing HIV infections among MSM and other populations, especially when adherence is high (Fonner et al., 2016). Initially, a reduction in HIV incidence of 44\% was found in the iPrEx study (Grant et al., 2010). Later, the IPERGAY and PROUD studies found reductions in HIV incidence up to 86\% (McCormack et al., 2016; Molina et al., 2015). In the IPERGAY study, it was found that PrEP is also effective for MSM when taken in an on demand regimen, with reductions in HIV incidence up to 97\% (Molina et al., 2015; Molina et al., 2017). In an on demand, or event-driven, regimen, PrEP 


\section{Chapter 1}

users only use PrEP at times when they expect a high risk of HIV. This regimen consists of two pills two to 24 hours in advance and one pill per day in the following two days.

Next to the effectiveness of PrEP, the clinical trials also assessed the safety of PrEP. A meta-analysis summarizing the results of 13 randomized clinical trials, including 15,678 participants, found no increase in severe renal or bone damage when using Truvada compared to a placebo (Pilkington et al., 2018). Even though side effects in renal function (such as creatinine elevations) were found to be limited and fully reversible (Pilkington et al., 2018), clinical guidelines recommend to frequently monitor renal function among PrEP users (World Health Organization, 2015).

The strong evidence for the effectiveness of PrEP in reducing HIV infections, together with the high safety, led to the development of guidelines to recommend PrEP for people at high risk of HIV, such as MSM (World Health Organization, 2014). According to the guidelines, MSM are eligible for PrEP if, for example, they have had a sexual transmitted infection (STI) in the past, or if they have condomless anal sex. Furthermore, the World Health Organization (WHO) indicated that "All men who have sex with men should have the opportunity to choose PrEP if they feel that it meets their HIV prevention needs" (World Health Organization, 2014). Despite these recommendations, PrEP was not immediately implemented on a large scale. An important barrier for PrEP implementation is the high price of PrEP. The price of Truvada is currently around $€ 500$ per month in Europe and $\$ 1600$ per month in the U.S. However, mathematical modeling studies show that PrEP is cost-effective, especially when MSM with high-risk behavior are targeted (Juusola et al., 2012; Kessler et al., 2014; Schneider et al., 2014). Also a modeling study on the situation in the Netherlands showed that PrEP is cost-effective, and even cost-saving when the price reduces and the effectiveness is high (Nichols et al., 2016).

With the high effectiveness of PrEP, the encouragement in WHO guidelines, and the promising results regarding cost-effectiveness, a rapid rollout of PrEP seems desirable. However, PrEP is not universally available, and the implementation of PrEP varies from country to country.

\section{Implementation of PrEP around the world}

The WHO formulated guidelines for the implementation of PrEP (World Health Organization, 2015), which were adopted by 40 countries (Hodges-Mameletzis et al., 2018), including the Netherlands (Hoornenborg \& Rijnders, 2016). However, despite having formulated na- 
tional guidelines on PrEP implementation, most of the countries do not provide formal access to PrEP services. The global advocacy organization AVAC maintains the website PrEPWatch. org including a 'global PrEP tracker' to provide information on the availability and accessibility of PrEP nation-by-nation (AVAC, 2020). A brief chronological overview of the introduction of PrEP in several regions is provided below, to highlight how PrEP was implemented in early adopting countries, and to outline the context that is relevant to the interpretation of findings of studies from different countries.

\section{United States}

The United States was the first country to take efforts in making PrEP available. The Food and Drug Administration (FDA) in the United States approved Truvada ${ }^{\circledR}$ for PrEP for the prevention of HIV infections in 2012 (Gilead, 2012; Roehr, 2012), taking the first step in making PrEP available outside of clinical trials. The Centers for Disease Control and Prevention (CDC) developed clinical guidelines for providing PrEP and PrEP became available via (free or co-payed) medication assistance programs and is covered by most national insurance plans (CDC, 2020; Kay \& Pinto, 2020). Initially, the uptake of PrEP was slow (Kirby \& Thornber-Dunwell, 2014), even among MSM who met the eligibility criteria for PrEP, (Parsons et al., 2017). Recently, PrEP uptake has been increasing, mostly among MSM in urban settings, for example in New York (Myers et al., 2018), San Francisco (Volk et al., 2020), and Chicago (Morgan et al., 2018). It is estimated that 200.000 people are using PrEP in the United States in 2020 (AVAC, 2020). Still, PrEP uptake should increase further, as the CDC estimated that there are 1.2 million eligible candidates for PrEP in the United States (Smith, Van Handel, et al., 2015).

\section{Australia}

Australia had a relatively rapid trajectory of implementing PrEP at a large scale. After small demonstration projects in 2014-2015 (Lal et al., 2017; Vaccher et al., 2017), PrEP became available via large-scale implementation projects in 2016 (Zablotska et al., 2018). As a result, PrEP use among eligible MSM increased between 2013 and 2017 from 2\% to 24\% (Holt, Lea, et al., 2018). In 2018, PrEP was approved for public subsidy in the Pharmaceutical Benefits Scheme (NPS MedicineWise, 2018), enabling further uptake of PrEP.

\section{Europe}

Four years after the approval of PrEP by the FDA in the United States, the European Medicines Agency (EMA) approved Truvada for PrEP (European Medicines Agency, 2016), there- 
by making PrEP legally accessible in the European Union (EU). In 2016, prior to the approval of PrEP, the attitudes towards PrEP were examined among 15,000 MSM in the Flash! PrEP in Europe study. About half of the MSM participants were interested in using PrEP and about 5\% had already used PrEP (AIDES, 2016). A year later, the EMIS-2017 study among 113,000 MSM in Europe found similar levels of interest in PrEP (44.4\%) and PrEP use (3\%) (European Centre for Disease Prevention and Control, 2019a). PrEP use was low, because PrEP was not formally available yet in most countries.

Across Europe, every country has to decide itself on the implementation of PrEP in the national health system. France was the first country in Europe to make PrEP available in January 2016, even before the approval of PrEP by EMA. This rapid PrEP implementation was driven by the successful results of the French IPERGAY trial (Molina et al., 2015) and the influence of strong community organization (Rojas Castro, Delabre, Morel, et al., 2019). England followed suit at the end of 2016 by making PrEP available in a National Health Service (NHS) trial for 10,000 individuals (Mayor, 2016). This temporary implementation of PrEP was criticized because it brought uncertainty about the long-term accessibility of PrEP for a larger group of people (Nagington \& Sandset, 2020). At the moment, most countries in Europe have not yet implemented PrEP in their healthcare system. According to data from the European Centre for Disease Prevention and Control (ECDC) from 2019, formal access to PrEP is only available in 16 of 53 reporting countries in Europe and neighboring countries (European Centre for Disease Prevention and Control, 2019b). In countries where PrEP is formally implemented and (partially) reimbursed, PrEP use increases rapidly, as studies in Belgium and Germany have shown (Mysior et al., 2020; Vuylsteke et al., 2019).

\section{The Netherlands}

The implementation of PrEP in the Netherlands started with the AMPrEP demonstration trial in 2015 (Public Health Service Amsterdam, 2015), providing initial access to PrEP to 376 MSM and transgender individuals. After the approval of Truvada for PrEP by the EMA in 2016, Truvada became available on prescription at Dutch pharmacies for approximately $€ 500$ per 30 pills. MSM in the Netherlands became aware of PrEP (Bil et al., 2015), but due to the limited capacity of AMPrEP and the high price of PrEP in pharmacies, PrEP was inaccessible for most MSM. The Dutch PrEP advocacy group PrEPnu lobbied for improving accessibility and affordability of PrEP. In 2017, the patent for Truvada for PrEP in the EU expired, and generic versions of PrEP became available. PrEP became more affordable for MSM in the Netherlands, as the price of generic PrEP became $€ 50$ per 30 pills (PrEPnu, 2017). In 2018, 
the Minister for Medical Care and Sport announced that PrEP would be included in the reimbursement scheme of the health insurance for 6500 people for the next five years, requiring a co-payment of the PrEP user of $25 \%$ of the costs (Ministry of Health, Welfare and Sport, 2018). In August 2019, PrEP provision started via the public health centers, and the costs of PrEP are (partially) reimbursed, making PrEP accessible to a larger group of MSM (Ministry of Health, Welfare and Sport, 2019).

\section{Informal PrEP use}

The timeline of the introduction of PrEP in the Netherlands shows that many years passed between the approval of PrEP and the introduction of national guidelines (in 2016) and the formal provision of PrEP in a reimbursement scheme (in 2019). During these years, the availability of PrEP was low and the price of PrEP was high, limiting the accessibility of PrEP for people who are at risk of HIV. Consequently, people tried to obtain PrEP via channels that are more affordable or readily available to them. Obtaining PrEP via unofficial channels has been labelled 'informal PrEP', and these channels mostly include clinics and (online) pharmacies in other countries where PrEP is available for a lower price, (Charpentier et al., 2014; Kurtz \& Buttram, 2016; Rosenthal et al., 2014; Zablotska et al., 2013). Also in their home country, MSM can obtain PrEP informally, by taking antiretrovirals (ARVs) that were prescribed as post-exposure prophylaxis (PEP) or as HIV treatment for HIV-positive individuals ("pill sharing”) (Zablotska et al., 2013).

It was expected that informal PrEP use would be equivalent to incorrect use of PrEP, because informal PrEP users may have misunderstandings of PrEP and do not receive PrEP counseling (Buttram, 2018; Dimitrov et al., 2013; Kurtz \& Buttram, 2016). The incorrect use of PrEP could then lead to drug resistance (Dimitrov et al., 2013). In practice, drug resistance is rare, especially when PrEP adherence is high (Parikh \& Mellors, 2016). In a qualitative study in England, informal PrEP users were found to be highly motivated early adopters who were well informed through community engagement (Paparini et al., 2018). Test results from an sexual health clinic in London showed that most informal PrEP users had similar drug plasma concentrations as formal PrEP users in clinical trials, indicating that PrEP adherence was high among informal PrEP users (X. Wang et al., 2018).

Informal PrEP use is mainly an issue in Europe, as most countries in Europe lack formal PrEP implementation. In Europe, varying by country, up to 67\% of PrEP users obtain PrEP via informal channels (European Centre for Disease Prevention and Control, 2019b). Given this 


\section{Chapter 1}

prevalence of informal PrEP use, it is important to investigate what the experiences of these informal PrEP users are, as they may differ from PrEP users in clinical trials.

\section{Challenges and opportunities related to PrEP access and uptake}

Given the improved availability of PrEP and the slow but steady increasing uptake of PrEP, questions arise regarding how PrEP should be implemented and how PrEP use can increase further to reach the maximum potential of PrEP in reducing new HIV infections. Determining who is interested in PrEP can help to identify under whom PrEP should be promoted. Furthermore, in the Netherlands, in a context where PrEP was not formally available, it is important to investigate what issues potential PrEP users face when trying to obtain PrEP.

\section{Interest in PrEP and PrEP uptake}

To reach the maximum potential of PrEP in reducing HIV infections at population level, it is important that the right persons start taking PrEP. On the one hand, MSM with high risk behavior who are eligible for PrEP should start using PrEP to reduce new HIV infections. On the other hand, MSM with low risk behavior who do not meet eligibility criteria, should not start using PrEP, in order to reach maximum cost-effectiveness. National guidelines provide eligibility criteria for PrEP use, but how does this translate into practice?

Studies in the United States have shown that there are disparities in the uptake of PrEP. Firstly, there is a gap between PrEP eligibility and PrEP uptake. It was found that while many MSM met the eligibility criteria for PrEP, only about $10 \%$ of them were using PrEP (Parsons et al., 2017). In the Netherlands, it is estimated that there are currently 3,500 individuals on PrEP (AVAC, 2020), of which 95\% are MSM (Staritsky et al., 2020), while it is estimated that 10,000 MSM are eligible candidates for PrEP (Hoornenborg, Krakower, et al., 2017; Reitsema et al., 2020). MSM may underestimate their own risk of HIV and may therefore not see themselves as eligible candidates for PrEP, inhibiting their intention to use PrEP (King et al., 2014; Rendina et al., 2017). Secondly, there are disparities in PrEP uptake between population subgroups. While Black and Latino MSM have an increased risk of HIV compared to white MSM (Hess et al., 2017), they are less likely to use PrEP (Bush et al., 2016; Kelley et al., 2015; Rolle et al., 2017). These findings are from a United States context and may not generalize to the situation of PrEP in the Netherlands.

To increase PrEP uptake among MSM, it is important to understand who is interested in PrEP. Determining what factors are related to interest in PrEP, may help to identify under whom PrEP implementation should be fostered. Before PrEP was available in the Nether- 
lands, a study among 448 MSM in the Amsterdam Cohort Study found that high-risk MSM (i.e. MSM who had condomless anal sex or had more than five casual sex partners in the past six months) and MSM with no steady partner were more interested in PrEP (Bil et al., 2015). This implies that PrEP interest was higher among MSM who could benefit most from PrEP. Since this study was conducted before the introduction of PrEP in the Netherlands, it is important to investigate current interest in PrEP, as the attitudes towards PrEP may have changed due to increased awareness.

\section{Accessing PrEP and PrEP-related healthcare}

After having identified who is interested in PrEP and who is willing to use PrEP, it is important to address how potential PrEP users access PrEP and PrEP-related healthcare. Especially when PrEP is limited available, or unaffordable, it is difficult to get hold of PrEP. In the Netherlands, PrEP can be obtained on prescription from the general practitioner. However, some general practitioners have a moralistic opinion about PrEP and refuse to prescribe PrEP (Leusink, 2020; Plomer et al., 2020). Furthermore, the high price of PrEP ( $€ 500$ per 30 pills in 2016 and 2017) was a hurdle for PrEP use in the Netherlands. Consequently, some MSM searched for PrEP through informal channels. A possible risk is that these informal PrEP users cannot maintain a constant supply of PrEP, because they do not have continuous access to PrEP points of sale (Rivierez et al., 2018). Having limited access to PrEP can lead to suboptimal use of PrEP and to increased risk of HIV (Buttram \& Kurtz, 2017).

In addition to access to PrEP, access to PrEP related healthcare is also relevant. Clinical guidelines recommend regular counseling, renal function testing, and HIV and STI testing for PrEP users (World Health Organization, 2015). When formal PrEP services are not implemented yet, or when MSM obtain PrEP informally, PrEP users may not receive regular counseling and testing: A study among 300 informal PrEP users in the United States in 2016 found that $27 \%$ of them were not making use of any medical care (Galea et al., 2016). Because of a lack of counseling, PrEP users may have misunderstandings of PrEP or may have increased risk-taking behaviors (Buttram, 2018; Dimitrov et al., 2013; Kurtz \& Buttram, 2016). Since the limited access to PrEP and the possible lack of counseling and testing may put informal PrEP users at risk for incorrect use of PrEP (and increased risk for HIV), it is relevant to investigate to what extent informal PrEP use occurs in the Netherlands and what the experiences of MSM in the Netherlands are regarding accessing PrEP related healthcare. 


\section{Behavioral changes following PrEP uptake}

While early research on PrEP focused on the effectiveness of PrEP and the implementation of PrEP, there is a need for psychosocial studies that can help to translate clinical findings into PrEP uptake (Auerbach \& Hoppe, 2015). PrEP may not only have an impact on sexual behavior, but also on attitudes towards sex and HIV. Promoting the psychosocial benefits of PrEP, such as increased feelings of empowerment and decreased stigma, may help to improve PrEP uptake (Grant \& Koester, 2016).

\section{Sexual risk behavior}

A much-debated topic around PrEP is the sexual risk behavior of PrEP users. Healthcare providers assumed that MSM who start using PrEP would use less condoms because they feel sufficiently protected against HIV by PrEP (Karris et al., 2014; Tellalian et al., 2013). During the first trials of PrEP, it seemed that condom use was increasing among PrEP users (Grant et al., 2010). An explanation for this increased condom use was that PrEP users received counselling in the setting of a clinical trial and were therefore more aware of the risks of HIV and STI and the usefulness of condoms. Later open-label and cohort studies showed that condom use decreased after PrEP initiation, as described in a meta-analysis and systematic review (Freeborn \& Portillo, 2018; Traeger et al., 2018). Condom use particularly declined among MSM who already used condoms inconsistently prior to PrEP use (Traeger et al., 2018).

A consequence of decreased condom use is that PrEP users may have an increased risk of getting other STIs. The Dutch demonstration trial AMPrEP found no increase in the number of STIs, but these participants already had a high prevalence of STIs prior to PrEP use (Hoornenborg et al., 2018). However, an increased risk of Hepatitis $C$ was found among PrEP users in this trial, mainly because there was an increasing overlap between the sexual networks of PrEP users and men living with HIV (Hoornenborg, Achterbergh, et al., 2017). A meta-analysis of open-label studies showed that more recent studies and studies with a longer follow-up time did show an increase in STIs among PrEP users (Traeger et al., 2018). Given the variety in findings of long-term studies, and the fact that these studies were conducted in clinical settings, it is important to investigate how PrEP users' sexual behavior develops in practice. Particularly in the case of informal PrEP use, when PrEP users may not receive counselling, there may be an effect of risk compensating behavior.

The concept of risk compensating behavior should not stand in the way of implementing PrEP. In the context of improvements in treatment and prevention of HIV, it is evident that 
risk compensating behaviors occur because people perceive less threat of HIV (Eaton \& Kalichman, 2007; Stolte et al., 2004). To mitigate increases in STIs as a result of risk compensating behaviors, the importance of behavioral counselling for PrEP users is stressed (Oldenburg et al., 2018; D. K. Owens et al., 2019). Instead of focusing on risk compensation that would hinder PrEP implementation, researchers have argued that the positive effects of PrEP on sexual health should be considered (Rojas Castro, Delabre, \& Molina, 2019).

\section{Psychosocial benefits of PrEP}

In addition to the potential HIV-preventive effect of PrEP, there may be other benefits of PrEP, such as psychosocial benefits. By identifying and promoting these psychosocial benefits, $\operatorname{PrEP}$ uptake may increase further. In a study among visitors of a sexual health clinic in Toronto it was found that decreasing fear of getting HIV was the second most common reason for willingness to use PrEP, after the reason of decreasing HIV risk (Rana et al., 2018). The reason of decreasing fear of getting HIV was not only mentioned by MSM with a high objective risk of getting HIV, but even more by MSM with a low objective risk of getting HIV. This indicates that PrEP can have psychosocial benefits even for MSM who are not necessarily eligible candidates for PrEP.

Many MSM experience fear of HIV infection (Koester et al., 2017; Prestage et al., 2012; Whitfield et al., 2019) and PrEP has the potential to reduce this HIV-related fear (Collins et al., 2017; Hojilla et al., 2016; Koester et al., 2017). By reducing fear of HIV, PrEP users may have more pleasurable sex, and consequently an increased quality of sex life (Mabire et al., 2019). Moreover, PrEP may empower PrEP users in their sexual decision making (UNAIDS, 2016), increasing their control over their sex life. For example, PrEP users can experiment with new sex practices that they previously did not dare because of fear of HIV, such as taking the receptive role in anal intercourse. A qualitative study in France found that PrEP users indeed express greater feelings of sexual freedom and increased sexual pleasure (Mabire et al., 2019). However, the amount of research into psychosocial benefits of PrEP is limited, and it remains to be investigated to what extent PrEP contributes to these effects. In addition, it is not investigated yet to what extent these improvements change over time, similar to changes in risk behavior over time.

Another dimension of increased sexual freedom is that PrEP users may feel more comfortable using recreational drugs. Drug use in a sexual context is also known as chemsex, and mainly includes the use of mephedrone, $\gamma$-hydroxybutyrate (GHB), $\gamma$-butyrolactone (GBL), and 


\section{Chapter 1}

crystallized methamphetamine (Crystal Meth/Tina) (McCall et al., 2015). A study among 4925 MSM visitors of the sexual health clinic in Amsterdam in 2016 found that $17.6 \%$ of MSM practiced chemsex in the past 6 months (Drückler et al., 2018). MSM who practiced chemsex were more often on PrEP (25.5\%) than MSM who did not practice chemsex (5.7\%) (Drückler et al., 2018). Chemsex is associated with an increased risk of HIV and STIs, because drug use can prolong sex sessions in which one has sex with multiple sex partners (Bourne et al., 2015). PrEP is a useful tool to reduce this risk of HIV, as condoms are not always used consistently during chemsex sessions (Roux et al., 2018). However, while PrEP might be beneficial in the context of chemsex, PrEP uptake is still limited among MSM who practice chemsex (Maxwell et al., 2019). The exact relationship between chemsex and PrEP should be more closely examined to encourage PrEP uptake among MSM who practice chemsex.

\section{Social stigma}

In the early years of PrEP the media readily spoke of 'Truvada whores' (Calabrese \& Underhill, 2015; Schwartz \& Grimm, 2017; Spieldenner, 2016).PrEP stigma is based on judgements about the sexual behavior of PrEP users (Dubov, Galbo, et al., 2018). For example, MSM associated PrEP use with condomless sex ("barebacking”) (Pawson \& Grov, 2018). As a result, MSM held PrEP users responsible for spreading STIs within the gay community. Moreover, if PrEP was seen as an alternative to condoms, PrEP was evaluated as 'irresponsible', because condoms have been associated with responsible sexual behavior (Haire, 2015). PrEP stigma has two possible consequences. Firstly, it creates a barrier for PrEP uptake. It was found that MSM who believed that PrEP is for promiscuous people are less interested in PrEP, despite the fact that they showed high-risk sexual behavior themselves (Eaton et al., 2017). Secondly, it may create a divide between PrEP users and MSM who are not using PrEP. PrEP users reported that they were sometimes rejected for sex because of their PrEP use (Franks et al., 2018). Data on PrEP stigma is not yet available in the Netherlands. Especially in a context of limited, but emerging PrEP availability, it is important to investigate whether PrEP-related stigma provides a hurdle for PrEP use.

In contrast to stigmatizing reactions and rejections for sex, PrEP can also have positive effects on sex partner selection. It was found that PrEP users can be seen as preferred sex partners, because of the HIV-preventive effect of PrEP, and consequently a lower transmission risk of HIV (Martinez \& Jonas, 2019). A study among 5,000 MSM in the United States found evidence for serosorting and 'PrEP sorting': MSM were more likely to have sex partners with the same HIV status and PrEP status (Grov et al., 2018). In addition, HIV-positive men were 
more likely to have sex with PrEP users than with HIV-negative men not on PrEP (Grov et al., 2018). This indicates that PrEP can play a role in bridging the serodivide between serodiscordant MSM. It was found that PrEP improves sexual experiences for both HIV-positive men and PrEP users, because of diminishing HIV stigma (Tester \& Hoxmeier, 2020).

HIV stigma is very common among MSM. In a study among 513 gay men living with HIV in the Netherlands, $70.2 \%$ reported experiencing stigma within the gay community (Stutterheim et al., 2008). HIV stigma has a negative impact on the wellbeing of gay men living with HIV as well as HIV-negative gay men (Courtenay-Quirk et al., 2006; Smit et al., 2012; Starks et al., 2013; Stutterheim et al., 2009). PrEP holds the potential of reducing HIV stigma, as PrEP may enable MSM to feel more comfortable having sex with people living with HIV (Brisson \& Nguyen, 2017; Grant \& Koester, 2016; Haas et al., 2017; Jaspal \& Daramilas, 2016; Malone et al., 2018; Persson, 2016). It must be examined to what extent PrEP can play a role in overcoming HIV stigma and improve sexual interactions between serodiscordant sex partners.

\section{Research questions}

This thesis aims to investigate the use of PrEP and the experiences of PrEP users in the Netherlands. This thesis addresses the following research questions:

1. What are the attitudes and usage intentions of MSM for PrEP in the Netherlands?

2. How do high-risk MSM in the Netherlands obtain PrEP and access PrEP-related healthcare?

3. What are the hurdles for PrEP use in the Netherlands?

4. What is the sexual behavior of PrEP users after PrEP initiation?

We investigate these questions in a context of limited, but emerging PrEP availability in the Netherlands. While PrEP use and sexual risk behavior have been investigated in the demonstration trial AMPrEP (Public Health Service Amsterdam, 2015), no studies have been conducted yet on (informal) PrEP use outside a clinical setting. The behavior of informal PrEP users may differ from the behavior of PrEP users in demonstration trials, as informal users do not always receive counseling. We examine sexual risk taking behavior, as well as the positive consequences of PrEP on sexual health. By investigating these aspects of PrEP use, we can inform community organizations and health care providers about optimal PrEP implementation. Tailored interventions can be derived to increase PrEP uptake among eligible MSM, to increase the maximum potential of PrEP to reduce new HIV infections. 


\section{Outline of the thesis}

In chapter 2 we first examine interest in PrEP. In the study in this chapter, we use data from the European research project 'Flash! PrEP in Europe'. This project was an initiative of the French community-based organizations AIDES and Coalition PLUS and was carried out jointly by 17 non-governmental organizations (NGOs) and two universities in twelve European countries. The aim was to assess PrEP usage and interest in PrEP among MSM on the eve of the introduction of PrEP in Europe in 2016. Various recruitment methods were used for the Dutch sample. This allowed us to investigate differences in PrEP interest among MSM who were recruited via various strategies. We specifically compared MSM from an LGBT research panel (AmsterdamPinkPanel, https://app.uva.nl/) with MSM who were recruited via convenience sampling. Furthermore, we investigated which factors were related to interest in PrEP and intention to use PrEP.

Chapter 3 describes the results of a qualitative study on the initial experiences of early PrEP users in the Netherlands. In 2018, we conducted interviews with informal PrEP users. At the time, generic PrEP was available in the Netherlands since a few months, making PrEP more affordable. As a result, informal PrEP users were able to switch from procuring PrEP via informal channels, to procuring PrEP at the local pharmacy on prescription. We explored the experiences of PrEP users in the context of this transition from informal PrEP use to formal PrEP access. We aimed to explore the experiences of these PrEP users as broadly as possible, particularly because PrEP use has not been investigated before in the Netherlands outside a demonstration trial. Therefore, we addressed three domains in this study. First, we investigated access to PrEP and PrEP-related healthcare. Second, we explored the responses PrEP users faced regarding their PrEP use, to assess possible PrEP stigma. We assessed responses from sex partners, as well as from friends and family. Third, we investigated the sexual behavior of PrEP users. Because participants in the Dutch demonstration trial AMPrEP were selected based on their high-risk behavior, we wanted to investigate the sexual behavior of other PrEP users in the Netherlands.

In chapter 4 we investigate predictors of PrEP uptake. We recruited participants via the website of the PrEP advocacy group PrEPnu (https://www.prepnu.nl/) over a period of two years (2017-2019). The website of PrEPnu provides information about using and obtaining PrEP aimed at potential PrEP users in the Netherlands. Participants received follow-up questionnaires after three and six months. At the first point of assessment, most participants did not yet use PrEP, but in the follow up questionnaires PrEP use was considerably higher. Therefore, 
we analyzed which behavioral and demographic characteristics on the baseline measurement predict PrEP uptake after six months. During the two years in which we recruited participants, there were changes in the accessibility of PrEP. Halfway through, in January 2018, generic versions of PrEP became available, reducing the price from $€ 500$ to $€ 50$ per 30 pills. We examined whether this price drop was related to increased PrEP uptake.

In chapter 5 we use the same dataset as in the previous chapter, and elaborate on the experiences of the participants who used PrEP. To investigate the role of PrEP in improvements in sexual health, we investigated the quality of sex life of PrEP users. We addressed several potential psychosocial and behavioral sequelae of PrEP, such as reduced fear for HIV, increased sexual freedom, and reduced condom use. In addition, we investigated how these attitudes and behaviors were related to quality of sex life.

In chapter 6 we examine the potential role of PrEP in reducing HIV stigma. We in particular investigated the attitudes of PrEP users who indicated to have sex with men living with HIV. We assessed their experiences of fear, comfort, and safety when having sex with men living with HIV. For this analysis the same dataset is used as in the previous chapters. We assessed whether the attitudes towards having sex with HIV positive men changes further over time after prolonged PrEP use.

Chapter 7 presents the general discussion. In this chapter the findings from the preceding studies are summarized, integrated, and connected to existing literature. After describing the main findings, the implications of these findings for the societal and scientific debates around PrEP are discussed. Subsequently, the strengths and limitations of this thesis are discussed. Finally, the directions for future research and the conclusions are discussed. 


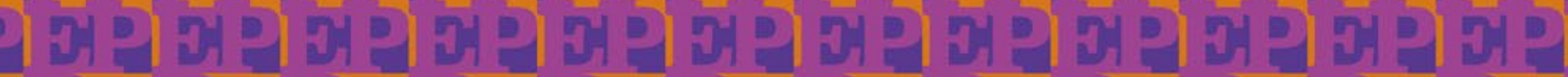

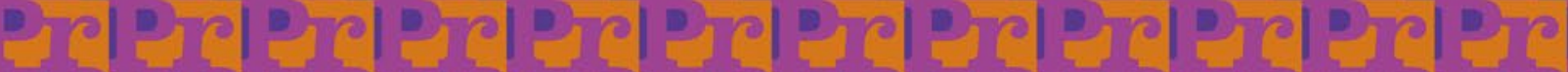

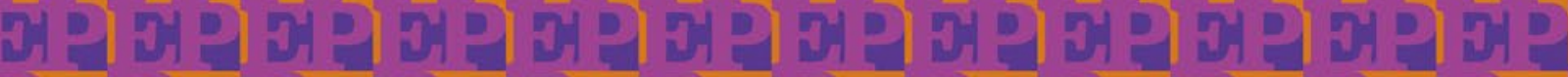

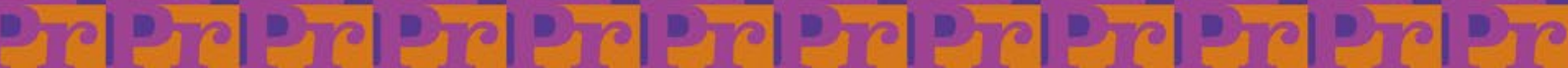

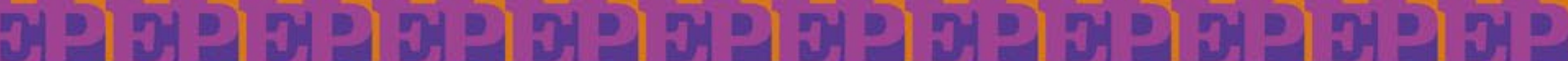

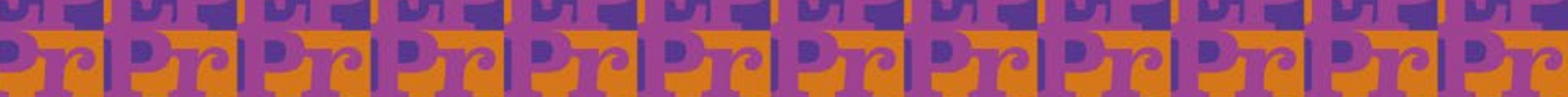

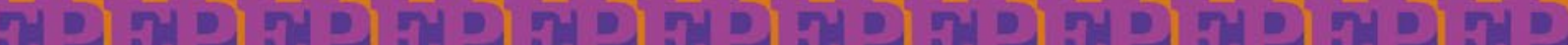

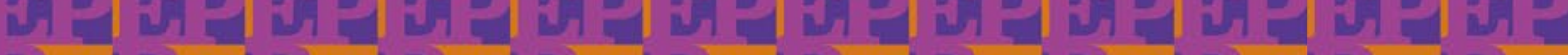

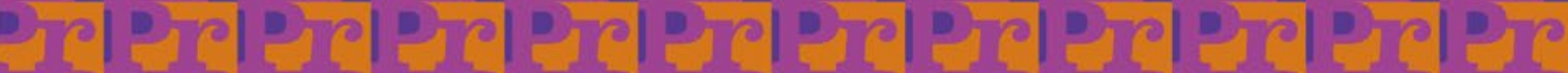

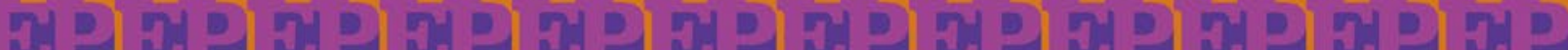

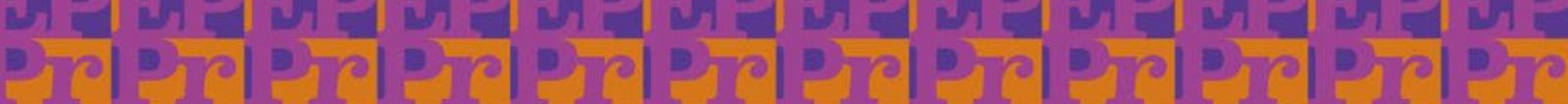

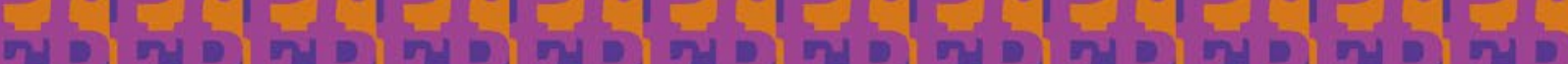
М

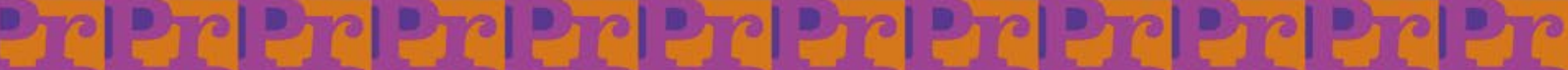
M

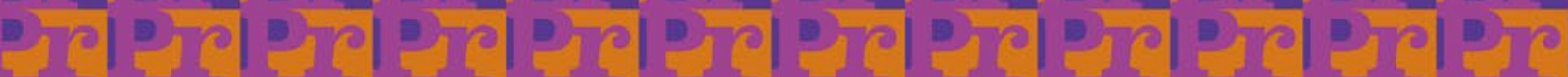

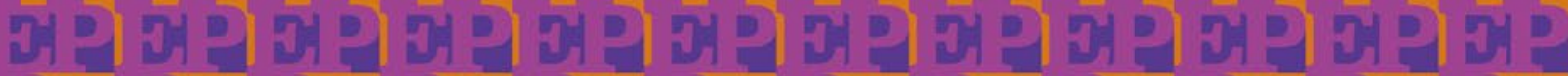

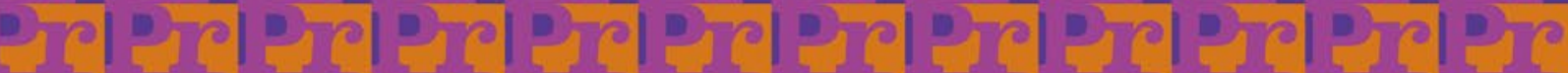

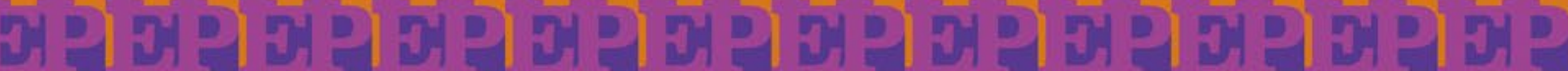

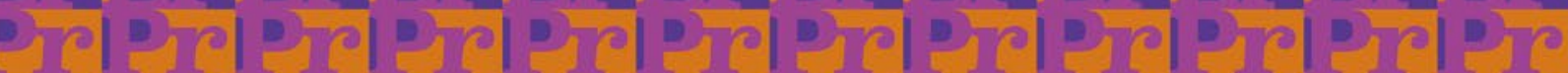

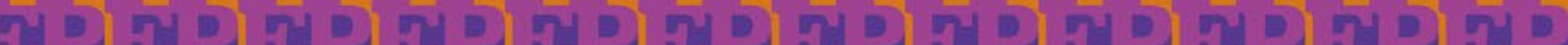

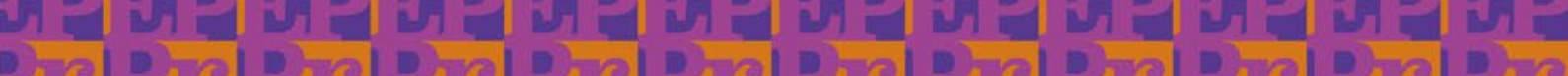

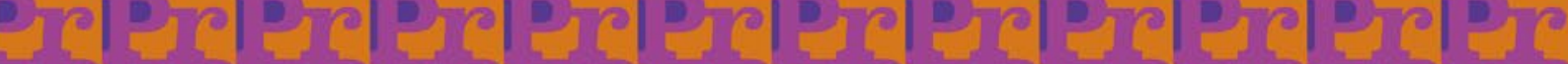

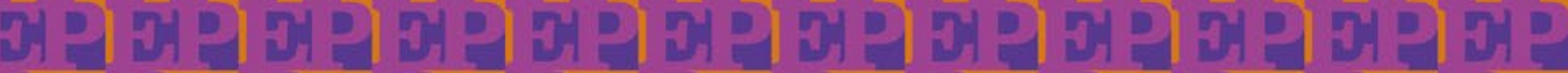

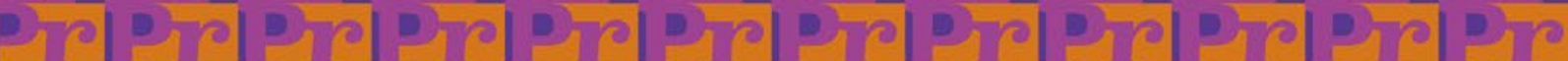

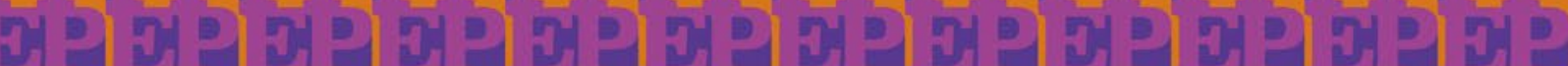

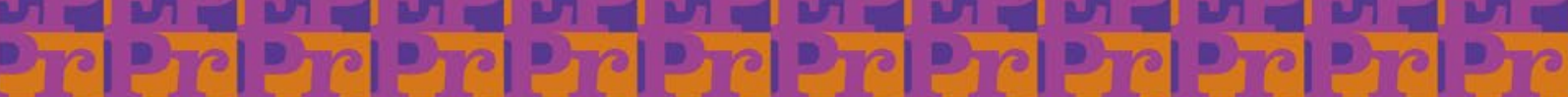

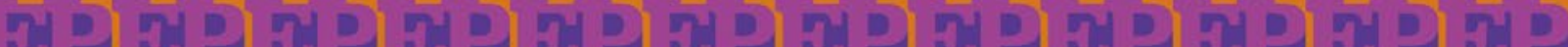

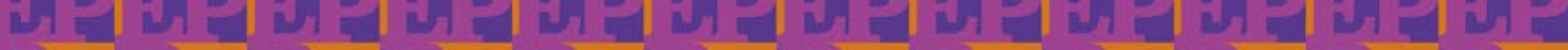

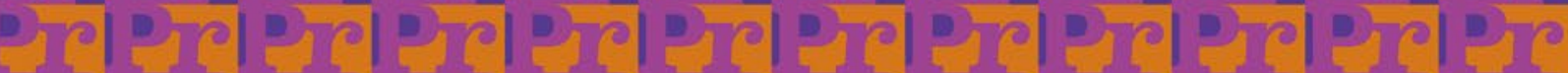

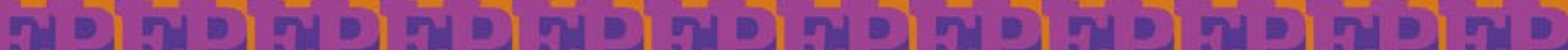
М

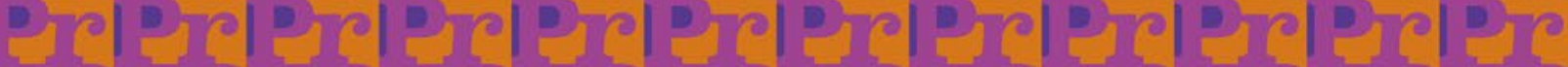
期

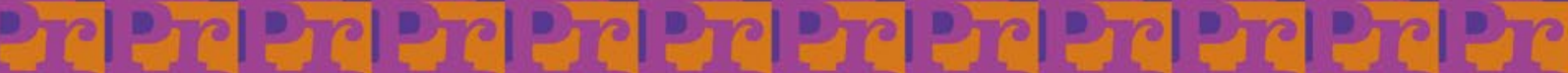

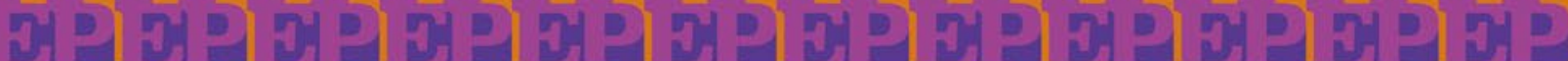

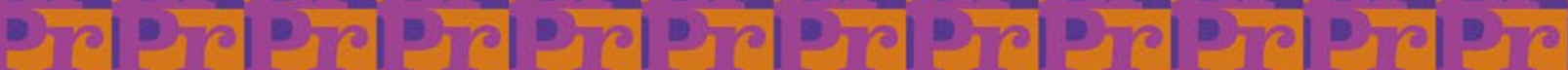




\section{CHAPTER 2}

\section{PrEP interest among MSM in the Netherlands: Covariates and differences across samples}

This chapter is based on:

Van Dijk, M., Duken, S. B., Delabre, R. M., Stranz, R., Schlegel, V., Rojas Castro, D., Bernier, A., Zantkuijl, P., Ruiter, R. A. C., De Wit, J. B. F., \& Jonas, K. J. (2020). PrEP Interest Among Men Who Have Sex with Men in the Netherlands: Covariates and Differences Across Samples. Archives of Sexual Behavior, 49(6), 2155-2164. https://doi.org/10.1007/s10508-019-01620-x 


\begin{abstract}
Despite increased availability of pre-exposure prophylaxis (PrEP), PrEP uptake has remained low. To promote uptake, factors related to PrEP interest among relevant target populations warrant investigation. The aim of this study was to provide an analysis of PrEP interest among men who have sex with men (MSM) in the Netherlands, while taking study recruitment strategies into account. We recruited 154 MSM from an LGBT research panel (AmsterdamPinkPanel) and 272 MSM from convenience sampling. Both samples were part of the Flash! PrEP in Europe survey and were compared on their PrEP interest, usage intentions, and sexual behavior. We conducted logistic regression analyses to discover variables associated with PrEP interest and intentions. Participants from the AmsterdamPinkPanel were less likely to use PrEP, had less knowledge of PrEP, and were less interested in PrEP than participants from convenience sampling. Significant covariates of PrEP interest were being single, more prior PrEP knowledge, sexual risk behaviors, such as not having used a condom during last sex and having ever used drugs in a sexual context, and not participating in the AmsterdamPinkPanel. Adding the recruitment strategy to the regression increased explained variance on top of predictors already described in the literature. Increased sexual risk behavior is related to increased PrEP interest and it helps to identify PrEP target groups. Recruitment strategies have a substantial impact on findings regarding PrEP interest and usage intentions. This study emphasizes the importance of using multiple strategies for recruiting participants to obtain a more comprehensive view of MSM's attitudes toward PrEP.
\end{abstract}




\section{Introduction}

Pre-exposure prophylaxis (PrEP) is an effective biomedical intervention to prevent HIV infection among HIV-negative individuals (Grant et al., 2010, 2014; Liu et al., 2013; McCormack et al., 2016; Molina et al., 2015). In the past years, the availability and accessibility of PrEP have improved. Four years after the approval of the Food and Drug Administration (FDA) in the United States in 2012 (Roehr, 2012), PrEP became formally available in Europe based on the approval of the European Medicines Agency (EMA) in 2016 (European Medicines Agency, 2016). Moreover, in many countries, PrEP has become more affordable through the inclusion of PrEP coverage in health insurance, such as in Belgium and Portugal (NAM Aidsmap, 2017), and the introduction of cheaper, generic versions of PrEP in some European countries (PrEPnu, 2017).

Despite the increased accessibility of PrEP, PrEP uptake has remained low, even among men who have sex with men (MSM) who are primary candidates for PrEP (Parsons et al., 2017). MSM may underestimate their risk of getting HIV, and may therefore see themselves as not requiring PrEP (Blumenthal et al., 2019; Parsons et al., 2017). Other barriers for PrEP uptake may include medical mistrust and anticipated stigma from sex partners (Biello et al., 2017; Cahill et al., 2017; Golub, 2018). To promote PrEP uptake, it is essential to further examine factors related to interest in PrEP in populations for whom PrEP implementation should be fostered.

Previous studies have shown substantial variation in PrEP interest. MSM at higher risk of getting HIV, because of a history of sexually transmitted infections (STIs) or multiple sex partners, were found to be more interested in taking PrEP (Bil et al., 2015; Golub et al., 2010; Yang et al., 2013). Further, some studies reported that younger MSM were more interested in taking PrEP (Aghaizu et al., 2013; Barash \& Golden, 2010), while other studies found that older MSM were more interested in taking PrEP (Yang et al., 2013); some studies did not find a relation between age and PrEP interest (Bil et al., 2015; Holt et al., 2012).

Variation in PrEP interest between studies can at least in part be explained by variation in how the question was formulated. Some studies assessed willingness to use PrEP (Barash \& Golden, 2010; Golub et al., 2013; Grov et al., 2016), while other studies combined measures of willingness to use PrEP and intention to use PrEP into one variable (Bil et al., 2015; Holt et al., 2012). Importantly, health risk research found that willingness and intention were related, but independent constructs. According to the prototype/willingness model, intentions are more 


\section{Chapter 2}

reflective thoughts in order to achieve a particular goal state, while willingness does not involve goal states, plans, or instrumental actions (Gibbons et al., 1998). Consequently, research has also found a clear distinction between willingness to use PrEP and intention to use PrEP (Rendina et al., 2017); MSM with a high intention to use PrEP had a lower education level, a lower income, a younger age, higher beliefs in the effectiveness of PrEP, saw themselves more often as appropriate candidates for PrEP, and felt greater partner pressure for condom nonuse, compared to MSM with a high willingness to use PrEP.

In addition, different recruitment strategies might also explain part of the variation in PrEP interest within MSM samples. For instance, in a cohort study in the Netherlands in 2012, $13.5 \%$ of MSM had a high intention to use PrEP and 60\% had a medium intention to use PrEP (Bil et al., 2015), while an online study in Scotland in the same period found a much lower intention to use PrEP (47.8\%; Frankis, Young, Flowers, \& McDaid, 2016). Differences in PrEP use and PrEP interest were also found in a study that directly compared different recruitment strategies: PrEP use and PrEP interest were higher among clients of an HIV/STI testing location compared to an online MTurk sample (Beymer et al., 2018).

The aim of the current study was to provide an in-depth analysis of PrEP interest among MSM in the Netherlands, while taking recruitment strategies into account. Our first research question was what the PrEP knowledge, PrEP use, PrEP interest, and PrEP usage intentions were among MSM in the Netherlands. Next, we investigated covariates of interest in PrEP and of intention to use PrEP. Our second research question was whether participants that were solicited via different recruitment strategies differed in PrEP interest and usage intentions. Specifically, participants in an LGBT research panel were compared to participants who were recruited online and at LGBT social venues. Finally, we compared characteristics of participants recruited through the LGBT research panel with LGBT research panel members not participating in this study to shed further light on underlying differences.

\section{Methods}

\section{Participants}

Dutch participants for the Flash PrEP in Europe (FPIE) survey were recruited via two recruitment strategies: (1) via an LGBT research panel (AmsterdamPinkPanel), or (2) via convenience sampling, through gay dating apps and websites (Hornet and PlanetRomeo), LGBT or MSM-related websites (for example, gay.nl), and 5000 flyers distributed in LGBT-themed 
bars, cafés, saunas, and STI clinics in June and July 2016. This provided the opportunity to compare sampling strategies, as FPIE participants stemming from the AmsterdamPinkPanel could be identified.

The AmsterdamPinkPanel is a partnership between COC Amsterdam (Dutch LGBT rights organization) and the Psychology Research Institute of the University of Amsterdam. The AmsterdamPinkPanel is a psychosocial LGBT research panel with more than 1400 members, of which 931 are MSM, with an age-range from 18 to 78 years. The members not only live in Amsterdam, but throughout The Netherlands. Sampling for the AmsterdamPinkPanel is community-driven and by self-enrollment.

Participants needed to be 18 years or older and to be HIV-negative or unaware of their HIV-status for inclusion in the current survey. Given the focus on MSM, only cisgender males were included in the analysis, defined by male gender at birth and current male gender. Participants were excluded from participation if they were HIV-positive, as PrEP is indicated only for HIV-negative individuals.

\section{Measures}

The FPIE survey was designed by the French community-based organizations AIDES and Coalition PLUS and adjusted by input from other non-governmental organizations and academics from 12 European countries. The 82-item questionnaire was the same in all participating countries and available in the local language. Translators chose the phrasing that seemed most adequate according to the populations targeted. A back-translation in English was conducted to ensure translation accuracy. For Dutch participants, the questionnaire was offered in Dutch and English. At the start of the survey, the only information provided about PrEP was that it is an HIV-prevention tool that is available in some countries around the world. We describe the measures relevant to the current research questions below. A full description of all measures in the FPIE survey can be found in the online supplementary material . The questionnaire was created in Qualtrics and the participants could not click back to previous questions.

\section{Sociodemographic characteristics}

In the FPIE survey, participants were asked to indicate their gender, age, relationship status, educational level, financial situation, country of birth, and country of residence. Gender was determined using two questions: gender at birth and current gender. Educational level was indicated by five levels, ranging from no higher education to $\mathrm{PhD}$ degree. Financial situation was assessed with a 6-point scale ranging from (1) "You can't make ends meet without bor- 


\section{Chapter 2}

rowing" to (6) "You are doing really well."

In the annual intake survey of the AmsterdamPinkPanel, some demographic questions were asked with different response options than the questions in the FPIE survey. In the AmsterdamPinkPanel educational level was indicated by seven levels, ranging from elementary school to university degree, and also included the options "rather not say" and "other education." Financial situation was indicated by self-reported yearly income in one of five different categories ( $<€ 30.000, € 31.000-€ 50.000, € 51.000-€ 75.000,>€ 75.000$ and "prefer not to say").

\section{Knowledge of PrEP}

Participants indicated if they were aware of PrEP (yes/no). If they did, they were asked to choose the correct definition out of five options to assess their knowledge. Two of the five definitions were correct ("PrEP is a pill that greatly reduces the risk of contracting HIV. You have to take it every day." and "PrEP is a pill that greatly reduces the risk of contracting HIV. You have to take it when you plan to have sex, before and two days after."), and a maximum of two choices were allowed. We defined knowledge of PrEP as correct if participants chose at least one of the correct definitions and none of the incorrect definitions. After these questions, all participants were provided with information about PrEP. This stated that PrEP provides protection against HIV when the drug is sufficiently present in the blood, but that it does not provide protection against other STIs and that it should not be confused with PEP (Post-Exposure Prophylaxis).

\section{PrEP use}

We asked participants if they were using PrEP. If so, they were not asked questions about interest in PrEP and intentions to use PrEP. Current PrEP users were asked how they obtained PrEP (e.g., via doctor's prescription, online purchase, or using HIV treatments prescribed as PEP).

\section{Interest in $\operatorname{PrEP}$}

To investigate willingness to use PrEP, we asked participants who did not use PrEP if they were interested in PrEP, using a 5-point scale, ranging from (1) definitely not interested to (5) definitely interested. Participants who indicated that they were (maybe) interested in PrEP were asked for their reasons why they were interested. They could express their agreement on the statements listed in Table 2 by using a 5-point scale, ranging from (1) strongly disagree to (5) strongly agree. Participants who indicated that they were not interested in PrEP were asked for their reasons why they were not interested. They could express their agreement on 
the statements listed in Table 3 by using a 5-point scale, ranging from (1) strongly disagree to (5) strongly agree. Participants could not add reasons themselves.

\section{Intention to use PrEP}

Participants' intentions to use PrEP were evaluated by asking if they intended to use PrEP when it becomes available, and before it becomes officially available within their countries' health care system. Both questions were rated on a 5-point scale, ranging from (1) definitely having no intention to (5) definitely having the intention to use.

\section{Sexual behavior}

We asked participants about the number of sex partners they had in the past six months and the frequency of anal sex, using a 5-point scale ranging from (1) never to (5) daily. Participants indicated if they used condoms during anal sex in the past six months by using a 5-point scale ranging from (1) never to (5) always.

\section{Subastance use}

Participants were asked if they used injectable drugs and if they used other drugs. If they responded yes to one of those questions, they were asked if they used drugs in a sexual context (yes/no).

\section{Data Analysis}

We analyzed the data using IBM SPSS Statistics version 24. We controlled for duplicate participation by checking IP addresses and response patterns on demographics and key variables. We used descriptive statistics to describe the sociodemographic characteristics of the sample, and undertook difference tests to compare subsamples of participants recruited through the AmsterdamPinkPanel and through convenience sampling. To investigate self-selection bias, we further assessed differences in the sociodemographic characteristics of the AmsterdamPinkPanel members who participated in FPIE with panel members who did not participate in the survey, based on intake data of the AmsterdamPinkPanel. We used descriptive statistics to describe the sample with respect to PrEP knowledge, use, interest and usage intentions, and assessed differences on these variables between AmsterdamPinkPanel participants and participants recruited through convenience sampling. For all difference tests we used analysis of variance (ANOVA) for continuous variables and chi-square tests for categorical variables. We used a multivariate analysis of variance (MANOVA) for comparing the answers on the multiple reasons for interest in PrEP, and reasons for disinterest in PrEP, between the participants recruited from the AmsterdamPinkPanel and the participants recruited through convenience 


\section{Chapter 2}

sampling. Given the most common type of variables, we conducted logistic regression analyses to assess variables associated with interest in PrEP and intention to use PrEP.

\section{Results}

\section{Participant Characteristics}

In total, 426 participants completed the FPIE survey. The mean age of the participants was 42 years, with age ranging from 18 to 75 years (Table 2.1). The Netherlands was the place of birth of 363 (85.2\%) participants. A bachelor degree or higher had been obtained by 290 (68.1\%) participants. The average score regarding perceived financial situation was $4.29(S D=1.24)$, indicating that participants perceived their financial situation between "all right" and "rather well." The relationship status of 191 (44.8\%) participants was single. We recruited 154 (36.2\%) males via the AmsterdamPinkPanel and 272 (63.8\%) males via convenience sampling. The AmsterdamPinkPanel participants and the participants recruited via convenience sampling differed on several sociodemographic characteristics. AmsterdamPinkPanel participants were on average older, perceived their financial situation as better, had a higher education level, and were more likely to be in a relationship (Table 2.1 ).

Furthermore, we could identify differences between AmsterdamPinkPanel participants and AmsterdamPinkPanel members who did not participate in the FPIE study. We invited all 931 male members of the AmsterdamPinkPanel to participate in the FPIE study, of whom 203 (21.8\%) decided to participate. The survey was completed by 154 participants. To answer our question whether there was a self-selection bias in MSM who responded to a survey about PrEP among research panel members, we compared AmsterdamPinkPanel members who participated in FPIE $(n=203)$ with AmsterdamPinkPanel members who did not participate $(n=728)$ on demographic variables. We observed differences in age and income, based on intake survey data: AmsterdamPinkPanel members participating in FPIE ( $M$ age $=52$, range: 18-81) were older than AmsterdamPinkPanel members who did not participate ( $M$ age $=46$, range: $\left.19-90 ; F(1,926)=29.14, p<.001, \eta_{\mathrm{p}}^{2}=.031\right)$. Of those who provided information on their income $(n=850)$, AmsterdamPinkPanel members participating in FPIE had a higher income than the AmsterdamPinkPanel members who did not participate, with $19.9 \%$ of the AmsterdamPinkPanel members participating in FPIE having an income below $€ 31.000$ compared to $33.1 \%$ of the AmsterdamPinkPanel members who did not participate $\left(\chi^{2}(4)=18.74\right.$, $p<.001)$. AmsterdamPinkPanel members overall are well educated, with a majority $(75.5 \%)$ having a Bachelor degree or higher. There were no differences in education level between the 


\begin{tabular}{|c|c|c|c|c|c|c|}
\hline & $\begin{array}{c}\text { Total } \\
(N=426)\end{array}$ & $\begin{array}{l}\text { Participants } \\
\text { from } \\
\text { Amsterdam } \\
\text { PinkPanel } \\
(n=154, \\
36.2 \%) \\
\end{array}$ & $\begin{array}{c}\text { Participants } \\
\text { from } \\
\text { convenience } \\
\text { sampling } \\
(n=272, \\
63.8 \%) \\
\end{array}$ & & $p$ & $\eta_{p}^{2}$ \\
\hline$M($ range / SD) & & & & $F(\mathrm{df})$ & & \\
\hline Age & $42(18-75)$ & $51(18-75)$ & $36(18-72)$ & $\begin{array}{c}133.64 \\
(1,424)\end{array}$ & $<.001$ & .24 \\
\hline $\begin{array}{l}\text { Perceived financial } \\
\text { situation }\end{array}$ & $4.29(1.24)$ & $4.75(1.09)$ & $4.03(1.24)$ & $\begin{array}{c}36.62 \\
(1,421)\end{array}$ & $<.001$ & .08 \\
\hline $\begin{array}{l}\text { Number of sex } \\
\text { partners in past } 6 \\
\text { months }\end{array}$ & $19(36.6)$ & $18.9(59.9)$ & $19.2(23.8)$ & $\begin{array}{c}.003 \\
(1,268)\end{array}$ & .95 & $<.01$ \\
\hline N (\%) & & & & $\chi^{2}(\mathrm{df})$ & & \\
\hline \multicolumn{7}{|l|}{ Education level } \\
\hline $\begin{array}{l}\text { No higher } \\
\text { education }\end{array}$ & $68(16.2 \%)$ & $17(11.2 \%)$ & $51(19.1 \%)$ & $40.81(4)$ & $<.001$ & - \\
\hline $\begin{array}{l}\text { Professional/ } \\
\text { vocational } \\
\text { education }\end{array}$ & $61(14.6 \%)$ & $14(9.2 \%)$ & $47(17.6 \%)$ & & & \\
\hline Bachelor degree & $138(32.9 \%)$ & $40(26.3 \%)$ & $98(36.7 \%)$ & & & \\
\hline Master degree & $118(28.2 \%)$ & $55(36.2 \%)$ & $63(23.6 \%)$ & & & \\
\hline $\mathrm{PhD}$ degree & $34(8.1 \%)$ & $26(17.1 \%)$ & $8(3.0 \%)$ & & & \\
\hline \multicolumn{7}{|l|}{ Relationship status } \\
\hline Single & $209(49.2 \%)$ & $56(36.4 \%)$ & $153(56.5 \%)$ & $26.11(2)$ & $<.001$ & - \\
\hline In a relationship & $123(28.9 \%)$ & $67(43.5 \%)$ & $56(20.7 \%)$ & & & \\
\hline $\begin{array}{l}\text { In an open } \\
\text { relationship }\end{array}$ & $93(21.9 \%)$ & $31(20.1 \%)$ & $62(22.9 \%)$ & & & \\
\hline $\begin{array}{l}\text { Had an STI in the } \\
\text { past } 12 \text { months }\end{array}$ & $80(20.3 \%)$ & $17(12.1 \%)$ & $63(24.8 \%)$ & $9.12(1)$ & .003 & - \\
\hline $\begin{array}{l}\text { Used a condom the } \\
\text { last time }\end{array}$ & $201(51.1 \%)$ & $69(49.3 \%)$ & $132(52.2 \%)$ & $.30(1)$ & .58 & - \\
\hline $\begin{array}{l}\text { Used drugs in a } \\
\text { sexual context }\end{array}$ & $136(33.2 \%)$ & $38(25.3 \%)$ & $98(37.7 \%)$ & $6.55(1)$ & .01 & - \\
\hline
\end{tabular}

AmsterdamPinkPanel members participating in FPIE or not $\left(\chi^{2}(6)=5.00, p=.54\right)$. AmsterdamPinkPanel members participating in FPIE were less often single (36.0\%) than other AmsterdamPinkPanel members $(44.8 \%)$, but there was no significant difference in relationship status between the two groups $\left(\chi^{2}(6)=9.17, p=.16\right)$. 


\section{Chapter 2}

\section{PrEP Knowledge and PrEP Use}

Out of the 426 FPIE participants, 383 (89.9\%) indicated that they were aware of PrEP. Of those who were aware of PrEP, 327 participants (85.4\% of those who were aware of PrEP) had correct knowledge of PrEP. Thirty-four participants (8.0\%) mistook PrEP for Post-Exposure Prophylaxis (PEP). AmsterdamPinkPanel participants were less likely to have correct knowledge about PrEP than the participants recruited through convenience sampling $(77.1 \%$ vs. $\left.89.7 \%, \chi^{2}(1)=10.93, p<.001\right)$.

Out of the 426 FPIE participants, 29 (6.6\%) used PrEP. AmsterdamPinkPanel participants used PrEP less often than the participants recruited through convenience sampling (3.2\% vs. $\left.8.8 \%, \chi^{2}(1)=4.82, p=.03\right)$. One participant received PrEP via a doctor's prescription. The remaining 28 participants received PrEP via a research trial, such as the Dutch AMPrEP trial (Hoornenborg et al., 2019).

\section{Interest in PrEP}

Out of 426 FPIE participants, 120 (28.2\%) were definitely interested in using PrEP, and 73 (17.1\%) participants were probably interested in using PrEP. There was a substantial difference in PrEP interest between participants recruited from the AmsterdamPinkPanel and the participants from convenience sampling $\left(\chi^{2}(4)=100.33, p<.001\right)$. The majority of participants recruited from the AmsterdamPinkPanel were not interested in using PrEP; 53 (36.1\%) of these participants were definitely not interested and 36 (24.5\%) were probably not interested. In contrast, the majority of the participants recruited through convenience sampling were interested in using PrEP; 106 (42.9\%) of these participants were definitely interested and 57 (23.1\%) were probably interested.

For those participants $(n=260)$ who were interested in using PrEP we assessed the reasons for their interest (Table 2.2). In a MANOVA, we found no significant difference in the reasons for interest in PrEP between AmsterdamPinkPanel participants and the participants recruited through convenience sampling $\left(F(6,253)=1.76, p=.11\right.$, Wilk's $\left.\Lambda=.96, \eta_{\mathrm{p}}^{2}=.04\right)$.

For those participants $(n=119)$ who were not interested in using PrEP we assessed the reasons for their lack of interest (Table 2.3). In a MANOVA, there was no significant difference in the reasons for lack of interest in PrEP between AmsterdamPinkPanel participants and the participants from convenience sampling $\left(F(10,108)=1.64, p=.10\right.$, Wilk's $\left.\Lambda=.87, \eta_{\mathrm{p}}^{2}=.13\right)$. 
Table 2.2. Mean scores on reasons for interest in PrEP for AmsterdamPinkPanel participants and the participants recruited through convenience sampling.

\begin{tabular}{lcccccc}
\hline & $\begin{array}{c}\text { Participants from } \\
\text { AmsterdamPink- } \\
\text { Panel }\end{array}$ & $\begin{array}{c}\text { Participants } \\
\text { from conveni- } \\
\text { ence sampling }\end{array}$ & & & & \\
& $M(S D)$ & $M(S D)$ & $F(1$, & & \\
& $N=58$ & $N=202$ & $258)$ & $p$ & $\eta_{p}^{2}$ \\
\hline I'd rather have condomless sex & $3.76(1.33)$ & $3.46(1.40)$ & 2.16 & .14 & .008 \\
I'm at risk of being infected by & $3.57(1.19)$ & $3.69(1.10)$ & .58 & .46 & .002 \\
HIV & & & & & \\
I would feel safer & $4.33(1.02)$ & $4.58(.76)$ & 4.23 & .04 & .016 \\
I would feel less anxious & $4.24(1.10)$ & $4.44(0.85)$ & 2.15 & .14 & .008 \\
I would feel more in control & $4.17(.96)$ & $4.36(.88)$ & 1.99 & .16 & .008 \\
I would have a more satisfying & $3.62(1.28)$ & $3.93(1.22)$ & 2.85 & .09 & .011 \\
sex life & & & & & \\
\hline
\end{tabular}

Note: The scores are on a 5-point Likert scale, with (1) for strongly disagree and (5) for strongly agree. Please note that in a MANOVA, no significant difference was found in the reasons for interest in PrEP between AmsterdamPinkPanel participants and the participants recruited through convenience sampling $\left(F(6,253)=1.76, p=.11\right.$, Wilk's $\left.\Lambda=.96, \eta_{\mathrm{p}}^{2}=.04\right)$.

\section{Intentions to use PrEP}

Regarding intention to use PrEP when it becomes officially available within the countries' health care system, we saw the same distribution of scores as for PrEP interest. Out of 426 FPIE participants, 119 (27.9\%) participants definitely had the intention to use PrEP when it becomes available and 81 (19.0\%) participants probably had the intention to use PrEP when it becomes available. The participants recruited through convenience sampling had a higher intention than AmsterdamPinkPanel participants $\left(\chi^{2}(5)=100.74, p<.001\right)$. Of the participants recruited through convenience sampling, 39.9\% definitely and $28.6 \%$ probably had the intention to use PrEP when it becomes available, compared to $13.4 \%$ and $6.7 \%$ of AmsterdamPinkPanel participants.

The intention to use PrEP before it becomes officially available was substantially lower than the intention to use PrEP when it becomes available. Only 26 (6.1\%) participants definitely and 37 (8.7\%) participants probably had the intention to use PrEP before it becomes available. Again, participants recruited through convenience sampling had a higher intention than AmsterdamPinkPanel participants $\left(\chi^{2}(5)=55.69, p<.001\right)$. Of participants recruited through convenience sampling, $9.3 \%$ definitely, and $12.1 \%$ probably had the intention to use PrEP before it becomes available, compared to $2.0 \%$ and $4.7 \%$ of AmsterdamPinkPanel participants. 
Table 2.3. Mean scores on reasons for non-interest in PrEP for AmsterdamPinkPanel participants and the participants recruited through convenience sampling.

\begin{tabular}{|c|c|c|c|c|c|}
\hline & $\begin{array}{c}\text { Participants } \\
\text { from } \\
\text { Amsterdam } \\
\text { PinkPanel } \\
M(S D) \\
n=82\end{array}$ & $\begin{array}{l}\text { Participants from } \\
\text { convenience } \\
\text { sampling } \\
M(S D) \\
n=37\end{array}$ & $F(1,117)$ & $p$ & $\eta_{p}^{2}$ \\
\hline $\begin{array}{l}\text { I don't want to take } \\
\text { medication every day }\end{array}$ & $4.38(1.22)$ & $3.84(1.46)$ & 4.39 & .04 & .036 \\
\hline $\begin{array}{l}\text { I don't want to pay for } \\
\text { PrEP }\end{array}$ & $2.67(1.31)$ & $2.65(1.27)$ & .01 & .93 & $<.001$ \\
\hline $\begin{array}{l}\text { I'm worried about the } \\
\text { side-effects }\end{array}$ & $3.93(1.15)$ & $3.70(1.27)$ & .91 & .34 & .008 \\
\hline $\begin{array}{l}\text { I'm afraid of being seen } \\
\text { in a negative light if I } \\
\text { take PrEP }\end{array}$ & $2.35(1.26)$ & $2.11(1.13)$ & 1.03 & .31 & .009 \\
\hline I don't believe it works & $2.09(1.19)$ & $2.57(1.30)$ & 3.95 & .05 & .033 \\
\hline $\begin{array}{l}\text { I'm worried of getting } \\
\text { other STIs }\end{array}$ & $3.26(1.52)$ & $3.51(1.54)$ & .72 & .40 & .006 \\
\hline $\begin{array}{l}\text { I don't need to change } \\
\text { how I protect myself }\end{array}$ & $4.39(.94)$ & $4.27(1.10)$ & .37 & .54 & .003 \\
\hline $\begin{array}{l}\text { I don't think I'm at risk } \\
\text { of being infected by } \\
\text { HIV }\end{array}$ & $3.77(1.18)$ & $3.19(1.41)$ & 5.43 & .02 & .044 \\
\hline $\begin{array}{l}\text { I don't want to undergo } \\
\text { regular medical check- } \\
\text { ups }\end{array}$ & $2.91(1.44)$ & $2.81(1.39)$ & .14 & .71 & .001 \\
\hline $\begin{array}{l}\text { I'm worried that I might } \\
\text { use condoms less often }\end{array}$ & $2.56(1.43)$ & $2.65(1.47)$ & .09 & .76 & .001 \\
\hline
\end{tabular}

Note: The scores are on a 5-point Likert scale, with (1) for strongly disagree and (5) for strongly agree. Please note that in a MANOVA, no significant difference was found in the reasons for lack of interest in PrEP between AmsterdamPinkPanel participants and the participants from convenience sampling $\left(F(10,108)=1.64, p=.10\right.$, Wilk's $\left.\Lambda=.87, \eta_{\mathrm{p}}^{2}=.13\right)$.

\section{Factors Associated with PrEP Interest and Intention to Use PrEP}

Table 2.4 shows the outcomes of the logistic regression of correlates of interest in PrEP. Significant multivariate correlates of PrEP interest were being single $(\mathrm{aOR}=.31,95 \% \mathrm{CI} .17-59$, $p<.001$ ), having correct prior PrEP knowledge (aOR $=1.96,95 \%$ CI 1.05-3.69, $p=.04$ ), not having used a condom for last sex $(\mathrm{aOR}=2.00,95 \% \mathrm{CI} 1.17-3.42, p=.01)$, having ever used drugs in a sexual context $(\mathrm{aOR}=2.68,95 \% \mathrm{CI} 1.52-4.73, p=.001)$, and not being a member of the AmsterdamPinkPanel ( $\mathrm{aOR}=.18,95 \% \mathrm{CI} .10-.34, p<.0001)$. Adding the variable "be- 
Table 2.4. Multivariable logistic regression examining correlates of interest in PrEP.

\begin{tabular}{|c|c|c|c|c|c|}
\hline & $B$ & S.E. & $p$ & $\mathrm{aOR}$ & $95 \% \mathrm{CI}$ \\
\hline Age & .01 & .01 & .55 & 1.00 & $.99-1.03$ \\
\hline \multicolumn{6}{|l|}{ Education level } \\
\hline No higher education & Ref & & & & \\
\hline Professional qualification & .59 & .45 & .19 & 1.81 & $.65-4.38$ \\
\hline Bachelor & .08 & .39 & .83 & 1.08 & $.51-2.31$ \\
\hline Master & .47 & .41 & .26 & 1.60 & $.71-3.59$ \\
\hline $\mathrm{PhD}$ & -.17 & .64 & .79 & .84 & $.24-2.94$ \\
\hline Financial situation & -.15 & .11 & .18 & .86 & $.69-1.07$ \\
\hline \multicolumn{6}{|l|}{ Relationship status } \\
\hline Single & Ref & & & & \\
\hline In a relationship & -1.16 & .32 & $<.001$ & .31 & $.17-.59$ \\
\hline In an open relationship & .07 & .34 & .83 & 1.08 & $.55-2.10$ \\
\hline STI in past 12 months $^{*}$ & .42 & .36 & .24 & 1.53 & $.76-3.08$ \\
\hline PrEP knowledge* & .68 & .32 & .04 & 1.96 & $1.05-3.69$ \\
\hline Not used a condom the last time ${ }^{\star}$ & .69 & .27 & .01 & 2.00 & $1.17-3.42$ \\
\hline Used drugs in a sexual context ${ }^{*}$ & .99 & .29 & .001 & 2.68 & $1.52-4.73$ \\
\hline Participant of AmsterdamPinkPanel ${ }^{*}$ & -1.71 & .33 & $<.001$ & .18 & $.10-.34$ \\
\hline
\end{tabular}

* The reference category for these variables is "no".

$\chi^{2}(13, N=358)=117.38, p<.001$, Nagelkerke $R^{2}=.37$

ing a member of the AmsterdamPinkPanel" to the regression model increased the proportion of explained variance by eight percent from $R^{2}=.29\left(\chi^{2}(12, N=358)=87.74, \mathrm{p}<.001\right)$ to $R^{2}$ $=.37\left(\chi^{2}(13, N=358)=117.38, p<.001\right)$.

Similarly, we conducted a logistic regression analysis regarding correlates of intention to use PrEP. The same multivariate correlates as for PrEP interest were found for intention to use PrEP, except for having correct prior PrEP knowledge ( $\mathrm{aOR}=1.40,95 \% \mathrm{CI} .75-2.62, p=.29$ ). Significant multivariate correlates of intention to use $\mathrm{PrEP}$ were being single (aOR $=.42,95 \%$ CI .23-.79, $p=.007)$, not having used a condom for last sex $(\mathrm{aOR}=1.88,95 \%$ CI 1.09-3.23, $p=.02)$, having ever used drugs in a sexual context ( $\mathrm{aOR}=2.90,95 \%$ CI 1.62-5.17, $p<.001)$, and not being a member of the AmsterdamPinkPanel (aOR =.13, 95\% CI .07-.25, $p<.001)$. Adding the variable "being a member of the AmsterdamPinkPanel" to the regression model increased the proportion of explained variance by 12 percent from $R^{2}=.27\left(\chi^{2}(12, N=356)=\right.$ $79.65, p<.001)$ to $R^{2}=.39\left(\chi^{2}(13, N=356)=121.91, p<.001\right)$. 


\section{Discussion}

The aim of this study was to provide an in-depth analysis of PrEP interest of MSM while taking recruitment strategies into account. It is important to examine factors related to interest in PrEP to be able to identify who can be targeted to increase PrEP uptake, because PrEP uptake has generally remained low, even among individuals with an elevated risk of an HIV infection (Parsons et al., 2017). Moreover, taking recruitment strategies into account is important since previous studies making use of different recruitment strategies have shown substantial variation in PrEP interest (Beymer et al., 2018; Bil et al., 2015; Frankis et al., 2016; Holt et al., 2012; Yang et al., 2013). For the current study, we recruited participants in two ways: via an LGBT research panel (AmsterdamPinkPanel) and via convenience sampling.

We found that recruitment strategies had a substantial impact on findings regarding interest in PrEP and intention to use PrEP. In particular, in the regression analysis, we found that adding recruitment strategy as a covariate substantially increased the proportion of explained variance of interest in PrEP and intention to use PrEP. We found that MSM participants recruited through the AmsterdamPinkPanel were less interested in PrEP, and had a lower intention to use PrEP than MSM participants who were recruited via convenience sampling. Also, AmsterdamPinkPanel participants were older, more educated, wealthier, and more often in a relationship compared to the participants from convenience sampling. Previous studies showed mixed results for the influence of sampling strategies on findings regarding interest in PrEP. Beymer et al. (2018), for example, reported higher interest in PrEP among visitors to an STI clinic compared to online participants, while Ferrer et al. (2016) reported lower interest among visitors to an STI clinic compared to online participants. Overall, the pattern of results across studies is diverse, confirming our notion that recruitment strategies and sample characteristics can play a major role in explaining different findings.

Overall, we found that $89.9 \%$ of the participants already knew what PrEP is. This is a large proportion compared to earlier studies (Bil et al., 2015; Frankis et al., 2016; Grov et al., 2016), suggesting that knowledge of PrEP is increasing over the years. Despite the high level of PrEP knowledge, we found low actual use of PrEP (6.6\%). This is comparable to findings of other studies in Europe (Bourne et al., 2019), and globally (Kamitani et al., 2018), and likely reflects the early stages of PrEP implementation. Nevertheless, about half of the participants (45.3\%) were interested in taking PrEP, and a similar number of participants (46.9\%) had the intention to use PrEP when available. This is in line with the majority of earlier studies, reporting a 
willingness to use PrEP among about 50\% of participants, as described in a review by Young and McDaid (2014). Covariates of interest in PrEP were being single, having correct prior PrEP knowledge, not having used a condom for last sex, having ever used drugs in a sexual context, and not being participant of the AmsterdamPinkPanel. The same covariates were found for intention to use PrEP, except for the covariate having correct prior PrEP knowledge. In contrast with the notion that there is a clear distinction between willingness to take PrEP and intention to take PrEP (Rendina et al., 2017), we did not encounter this distinction in our results. Because PrEP had only limited availability in the Netherlands at the time of our study, PrEP use was fairly distant for most MSM and, therefore, it may not have been possible to find this fine distinction between interest in PrEP and intention to use PrEP, as was found in the U.S.-based study of Rendina et al. (2017).

To investigate the possibility of a self-selection bias in MSM who respond to a survey about PrEP, we compared AmsterdamPinkPanel members who participated in FPIE with AmsterdamPinkPanel members who did not participate. We found that MSM from the AmsterdamPinkPanel who participated in the FPIE survey were older and more affluent than MSM from the AmsterdamPinkPanel who did not participate. This finding is surprising since we expected that younger MSM would be more likely to respond to the questionnaire, because they are more interested in taking PrEP according to previous studies (Holt et al., 2012). However, the majority of the AmsterdamPinkPanel participants (60.6\%) were not interested in using PrEP, which may have influenced their motivation to take part in the survey and to voice their views.

A limitation of our study is that, while we recruited participants via different strategies, inclusion was based on convenience sampling. Random population sampling strategies, in which each individual in the population has the same probability of being included, seem to be less affected by self-selection bias than nonprobability samples (Meyer \& Wilson, 2009), and LGBT probability samples are found to differ from LGBT participants in nonprobability community samples. LGBT nonprobability community participants were younger, had more often an exclusive same-sex orientation, and were more open about their sexual orientation (Kuyper et al., 2016). They also reported more high-risk sexual behavior (Dodds et al., 2006; Evans et al., 2007). However, probability samples for LGBT participants are expensive to establish, and most studies on PrEP use and PrEP interest are based on nonprobability samples. The LGBT population makes up a small fraction of the general population, requiring the recruitment or screening for inclusion of many people ineligible for participation to be able to 


\section{Chapter 2}

include a sufficient amount of LGBT participants (Meyer \& Wilson, 2009). However, a recent study, conducted in the United States in 2016, made use of data from a probability sample to examine PrEP use and familiarity with PrEP among MSM (Hammack et al., 2018). They found that $4.1 \%$ of the respondents had used PrEP, and that $59.8 \%$ was familiar with PrEP. This level of PrEP use is comparable to the online nonprobability samples in the study of Beymer et al. (2018), who conducted the study in roughly the same period (2015-2016).

A further limitation is that we could only compare AmsterdamPinkPanel participants with the participants from convenience sampling, but could not make further distinctions within the latter group. We could not track the recruitment source of the convenience sampling participants, because this was not a primary goal of the FPIE survey. For example, we could not compare participants recruited through gay dating apps with participants recruited through gay social venues. This is a drawback as it is expected that MSM who use gay dating apps have different sexual behaviors than MSM who are not using such apps (Lewnard \& Berrang-Ford, 2014). Another limitation is that the results were based on participants' self-reports, which may be affected by reporting bias, as opposed to clinical data such as measuring intracellular PrEP drug levels. However, as participants filled out the questionnaire anonymously, social desirability bias is expected to be limited.

For future research, we recommend that researchers not only focus on obtaining an MSM sample per se, but to carefully consider the characteristics of the sample they recruit, and how this can influence their findings. Having clear inclusion and exclusion criteria, and drawing on different sample sources and recruitment strategies, will help to identify and address potential sampling biases. A strength of our study is that we could compare two samples that were recruited at the same time, eliminating the influence of timing. This is important because PrEP is relatively new and community awareness and accessibility are increasing.

In conclusion, while findings show differences between samples according to recruitment strategies, overall findings suggest that PrEP knowledge is high among participating MSM, but PrEP use is low. About half of the participants were interested in using PrEP, and findings regarding covariates of interest in PrEP and intention to use PrEP provide important directions for the promotion of PrEP. Promotional activities may in particular target MSM who are single, do not always use condoms, and use drugs in a sexual context. Promoting PrEP among these MSM may be especially pertinent to increase PrEP uptake. 
PrEP interest among MSM in the Netherlands 


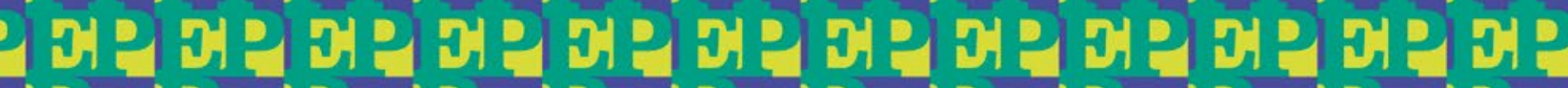

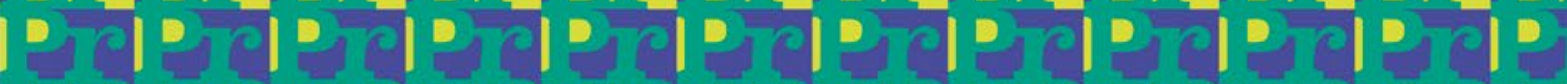

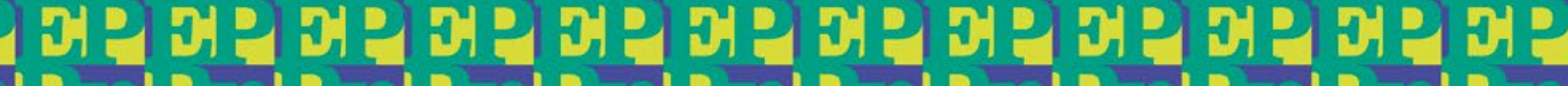

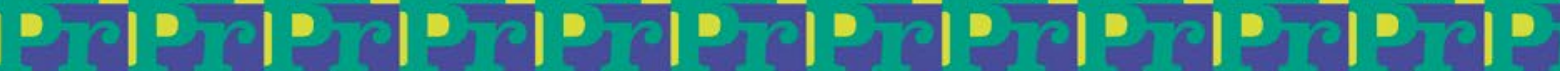

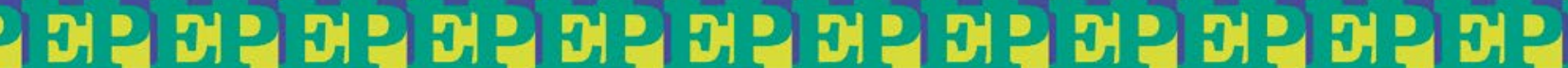
$\begin{array}{lll}0 & 0\end{array}$

斯

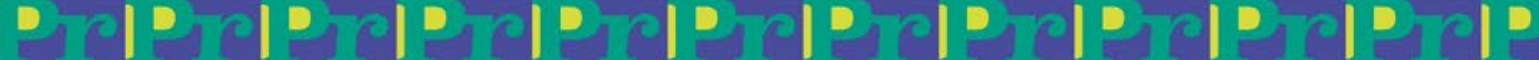

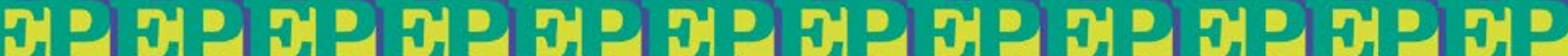

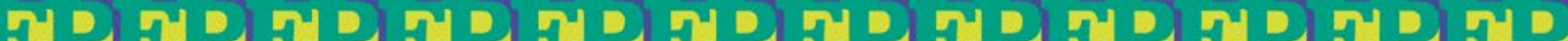

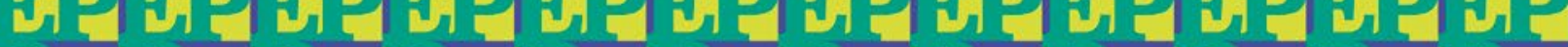
D

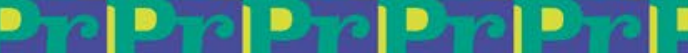

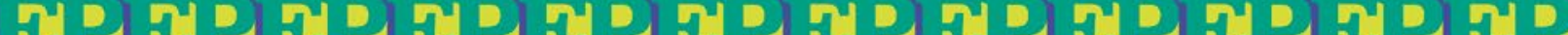
עद

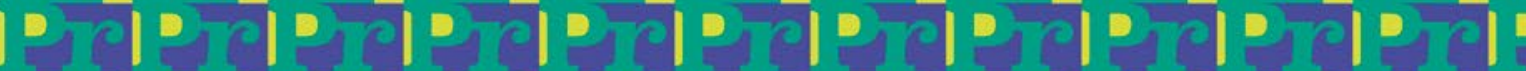

If (1)

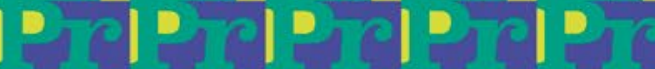
a

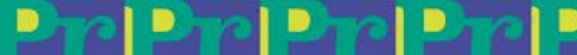

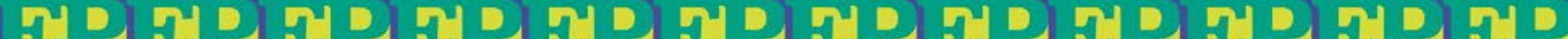
|

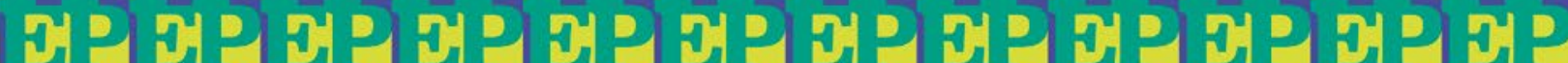

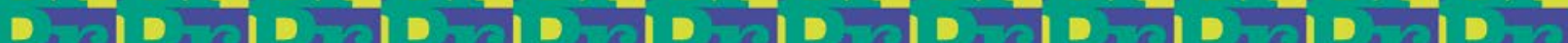

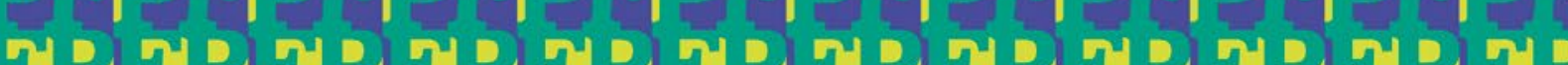

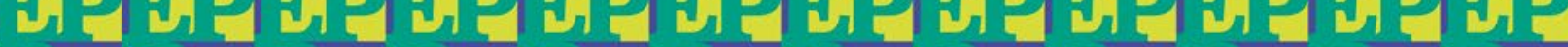

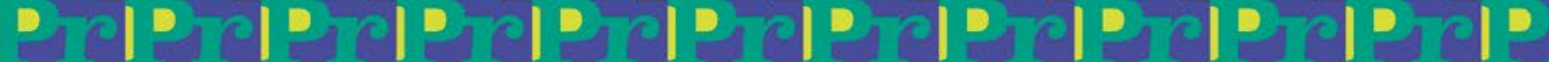

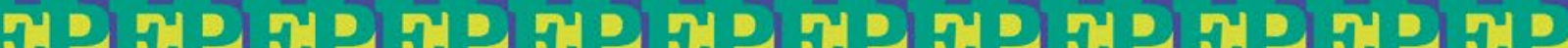

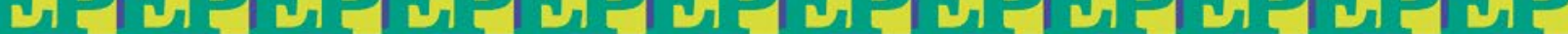

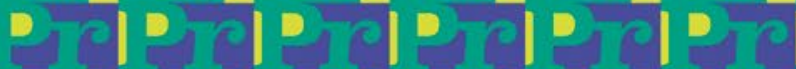

N D Dis

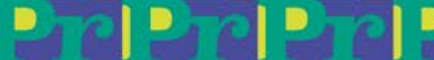

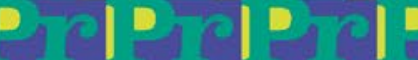

If

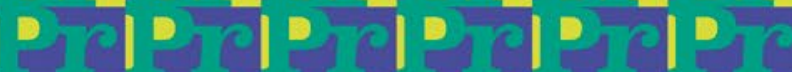

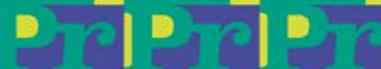
D.

ע (

D)
D) D 1010 D.

ע

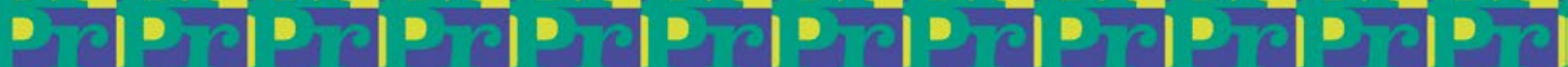

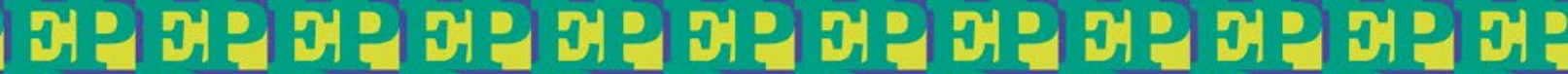




\section{CHAPTER 3}

\section{Socio-sexual experiences and access to healthcare among informal PrEP users in the Netherlands}

This chapter is based on:

Van Dijk, M., De Wit, J. B. F., Kamps, R., Guadamuz, T. E., Martinez, J. E., \& Jonas, K. J. (2020). Socio-Sexual Experiences and Access to Healthcare Among Informal PrEP Users in the Netherlands. AIDS and Behavior. https://doi.org/10.1007/s10461-020-03085-9 


\begin{abstract}
The aim of this qualitative study was to explore the experiences of informal PrEP users regarding access to PrEP and PrEP-related healthcare, community responses, sexual behavior and well-being. We interviewed 30 men who have sex with men (MSM) in semi-structured online interviews between March and August 2018. Interviews were analyzed using interpretive description. Informal PrEP users were well informed about the use of PrEP, but sometimes did not make use of renal testing. Participants reported a lack of PrEP knowledge among healthcare providers, which limited their access to PrEP and put them at risk, as they received incorrect information. Although some participants reported negative reactions from potential sex partners, most received positive reactions and were sometimes seen as more desirable sex partners. PrEP healthcare services should not only be accessible to formal PrEP users, but also to PrEP users who procure PrEP informally.
\end{abstract}




\section{Introduction}

Despite improvements in the availability of pre-exposure prophylaxis (PrEP), there is still a large gap between PrEP access and expressed need for PrEP in many countries around the world (Hayes et al., 2019; Weiss et al., 2018; Zablotska et al., 2016). According to data from the European Centre for Disease Prevention and Control (ECDC) from 2019, formal access to PrEP is available only in 16 of 53 reporting countries in Europe and neighboring countries (European Centre for Disease Prevention and Control, 2019c). Access to PrEP has been limited due to structural barriers such as the high costs of PrEP, but also by a lack of PrEP implementation in existing healthcare settings (Arnold et al., 2017; Dubov et al., 2019; Golub et al., 2013; Hannaford et al., 2018; Rice et al., 2019). As a consequence, some men who have sex with men (MSM) obtain PrEP via channels that have been labelled "informal”. These informal channels include online pharmacies, pharmacies and clinics in other countries where generic PrEP is sold at a cheaper price point, via post-exposure prophylaxis (PEP) treatments, and via pill sharing of HIV-positive friends who take antiretroviral therapy (Charpentier et al., 2014; Kurtz \& Buttram, 2016; Rosenthal et al., 2014; Zablotska et al., 2013). In Europe, varying by country, up to $67 \%$ of PrEP users obtain PrEP via informal channels (European Centre for Disease Prevention and Control, 2019c). Informal PrEP use has been associated with suboptimal regimens and dosing of PrEP, a lack of knowledge about PrEP, and a lack of uptake of the recommended health services, such as regular HIV and renal testing and behavioral counseling (Buttram, 2018; Dimitrov et al., 2013). Inconsistent access to PrEP and PrEP-related health services, combined with the potentially suboptimal use of PrEP, puts informal PrEP users at risk for HIV infection and other health risks (Buttram, 2018).

Most studies to date have investigated PrEP use in the context of clinical trials or in formal healthcare settings (Grant et al., 2010; Hojilla et al., 2016; Holt, Lea, et al., 2018; Hoornenborg et al., 2019; Lal et al., 2017; Liu et al., 2013; McCormack et al., 2016; Milam et al., 2019; J. M. Molina et al., 2017; Sagaon-Teyssier et al., 2016). However, because the availability of formal PrEP healthcare is limited in many countries (European Centre for Disease Prevention and Control, 2019c), there is a need to study PrEP use in such contexts of limited availability, reflecting a real world setting that for many imply informal use. Moreover, to optimize PrEP implementation, it is needed to understand how MSM comprehend and experience PrEP, thereby taking their psychological and social realities into account (Auerbach \& Hoppe, 2015). Informal PrEP use comes with specific challenges that cannot be observed in clinical trials, such as how PrEP is obtained and how to communicate with healthcare providers who 
are less informed about PrEP. So far, only a few studies have been conducted on informal PrEP use, mainly focusing on obtaining PrEP, using PrEP, and receiving medical support (Buttram, 2018; Paparini et al., 2018; Rivierez et al., 2018; Zablotska et al., 2013). While sexual risk behavior, including risk compensation, is often studied in the context of formal PrEP use (Hojilla et al., 2016; Holt, Lea, et al., 2018; Hoornenborg et al., 2019; Lal et al., 2017; Liu et al., 2013; Milam et al., 2019; Sagaon-Teyssier et al., 2016), this is underexplored in the context of informal PrEP use.

While informal PrEP use typically refer to "non-prescribed" or "off-label" PrEP use (Brisson et al., 2019), focusing on the procurement of PrEP, it is also important to consider how informal PrEP users access the appropriate medical supervision indicated with respect to $\mathrm{PrEP}$ use (i.e. CDC-guidelines (CDC, 2017)). A broader definition of informal PrEP use is hence the "non-prescribed and non-medically supervised" PrEP use (Buttram, 2018). This broader definition is particularly appropriate in the context of the Netherlands, and other countries where (generic) PrEP can be obtained before formal access is realized and formal PrEP-related medical supervision services are made available. The aim of this study was to explore the experiences of informal PrEP users in a context of the transition to full formal PrEP access. More specifically, we investigated the experiences of informal PrEP users across three domains: Access to informal PrEP and PrEP-related healthcare, and perceived community responses to PrEP use, and PrEP users' sexual behavior and well-being.

\section{Access to PrEP and PrEP-related healthcare}

Compared to the formal PrEP use, informal PrEP use may be challenging, as PrEP may not be easily obtained, and PrEP-related healthcare may often not be accessible. Obtaining PrEP and maintaining a consistent supply of PrEP is reported as a common difficulty for informal PrEP users (Rivierez et al., 2018), often resulting in the use of a regimen that requires less pills, for example an on demand regimen (Koppe et al., 2019). PrEP users may also be forced to take less pills than the recommended dosage. Nevertheless, a study among informal PrEP users in London found that despite difficulties in obtaining PrEP, most informal PrEP users had similar drug plasma concentrations as formal PrEP users in clinical trials (X. Wang et al., 2018). In addition, informal PrEP users have to arrange the recommended counseling and three-monthly HIV, STI and renal function tests themselves, and they may also have to pay for these services out-of-pocket. Research has found that most of the informal PrEP users do not access PrEP-related healthcare, and do not regularly test for HIV (AIDES, 2016). Those who do access PrEP-related healthcare may be confronted with healthcare providers who may 
reject or stigmatize them for their informal use, or have a lack of knowledge or moralistic opinions about PrEP (Brisson, 2018; Leusink, 2020; Schwartz \& Grimm, 2019). For example, healthcare providers were worried about risk compensating behavior (Karris et al., 2014; Tellalian et al., 2013), and medical students were less likely to prescribe PrEP to patients with the highest risk behaviors (Calabrese et al., 2018), indicating that personal assessments of the healthcare provider play a role in the decision to prescribe PrEP or to offer medical supervision to PrEP users.

\section{Community responses}

PrEP users may face stigmatizing reactions regarding their PrEP use, and have been described in the media as "Truvada whores" (Calabrese \& Underhill, 2015; Schwartz \& Grimm, 2017). Among MSM, and even among PrEP users themselves, stigmatizing reactions are also noted (Puppo et al., 2020). For example, gay and bisexual men have been found to associate PrEP use with "barebacking" (intentional condomless anal intercourse), and to hold PrEP users responsible for the spread of STIs in the gay community (Pawson \& Grov, 2018). PrEP users also report feeling stigmatized as a result of being rejected for sex dates or by being seen as HIV positive (Franks et al., 2018). On the other hand, PrEP users can also be preferred as sex partners by MSM who are not using PrEP, as they are potentially at lower risk of transmitting HIV (Martinez \& Jonas, 2019). PrEP users have also been seen as more responsible and trustworthy, and their use of PrEP was not itself perceived as a reflection of sexual promiscuity (Martinez \& Jonas, 2020). Given these mixed findings regarding PrEP-related stigma, it is important to further explore the responses that informal PrEP users face from other MSM and potential sex partners. We did not expect differences in the perceptions of informal and formal PrEP users per se. It could however be that informal PrEP users are evaluated as having a poor "PrEP citizenship" (Orne \& Gall, 2019) compared to formal PrEP users, as they may not have a reliable PrEP supply or are not being continuously monitored on HIV and STI testing.

\section{Sexual behavior and well-being}

Sexual behaviors of PrEP users have mainly been studied in the context of formal PrEP use, in particular in the context of clinical trials and demonstration projects, such as ANRS-IPERGAY (J.-M. Molina et al., 2015), AMPrEP (Hoornenborg et al., 2018), iPrEx (Grant et al., 2010), and PROUD (McCormack et al., 2016). However, clinical trials may give a biased view of changes in sexual behavior, because of the selection of participants (i.e. selected participants are MSM with (extreme) high risk behavior) or because of the included behavioral counseling, that may be absent in a real-world situation. Therefore, we also explored the sex- 
ual behavior of informal PrEP users in this study. A commonly reported change in sexual behavior after initiation of PrEP use is an increase in condomless anal intercourse (Hoornenborg et al., 2018). Studies of formal PrEP use have also highlighted reduced fear of HIV, and improved sexual satisfaction and well-being (Collins et al., 2017; Hojilla et al., 2016). Similar findings have been obtained in studies of PrEP in non-clinical settings as well as in relation to informal PrEP use, including decreased condom use, reduced fear of HIV, and improved quality of sex life (Keen et al., 2020; Paparini et al., 2018; Prestage et al., 2019). Informal PrEP use has also been associated with other increases in risk behavior, including recreational drugs use and group sex (Zablotska et al., 2013).

\section{The current study}

The current study explored the behaviors and experiences of informal MSM PrEP users in the Netherlands. In the Netherlands, some MSM started to use PrEP informally before PrEP became formally available through Dutch pharmacies in 2016. Even after PrEP became formally available, informal procurement of PrEP continued, because PrEP was initially not affordable for many if not most MSM ( $€ 500$,- per 30 pills). At the time, affordable PrEP in the Netherlands was only formally available through the AMPrEP study, a demonstration project that provided PrEP for free to 376 participants (Hoornenborg et al., 2019). In November 2017, the price of PrEP in the Netherlands dropped significantly (to $€ 50$,- per 30 pills) when generic versions became available in pharmacies in the Netherlands (PrEPnu, 2017). Although formal PrEP guidelines have been available in the Netherlands since 2016 (Hoornenborg \& Rijnders, 2016), the practical implementation of PrEP was delayed as healthcare providers waited for an implementation policy of the Ministry of Health that addressed the provision of PrEP-related medical care. This PrEP implementation policy came into effect only in August 2019 (Ministry of Health Welfare and Sport, 2018, 2019). In other words, while PrEP users were able to obtain PrEP in the Netherlands as of 2016, it took another three years until arrangements of medical supervision of PrEP use were in place.

Investigating the experiences of informal PrEP users can inform healthcare professionals and community support groups about the challenges that informal PrEP users face. This investigation can provide guidance for necessary actions to provide PrEP related healthcare for informal PrEP users, and how to potentially bring them into formal PrEP care. Furthermore, our study also aims to corroborate previous findings of effects of formal PrEP use, such as on sexual well-being, as the type of procurement and use should not impact on the perceived benefit of PrEP. 


\section{Methods}

\section{Participants and recruitment}

In a survey that was conducted through the website of the Dutch PrEP advocacy group PrEPnu (www.prepnu.nl), 64 MSM indicated using PrEP informally. We invited these MSM for an interview, of which 26 (40.6\%) agreed. In addition, four participants were recruited via peer referral, resulting in a total sample of $\mathrm{N}=30$. The participants did not receive any compensation for participation in the interview. The study was approved by the Ethical Review Committee of the Faculty of Psychology and Neuroscience, Maastricht University (code ERCPN 188_05_02_2018).

\section{Procedure}

Between March and August 2018, 30 semi-structured interviews were conducted using an instant messaging program (Skype or WhatsApp, depending on the preference of the participant). We used instant messaging, because this allowed us to reach MSM from all over the country without being restricted by travel time and costs. In addition, because of the sensitive nature of the topics under investigation, we expected that MSM would express themselves more freely in an online chat interview compared to a (online) face-to-face interview, as anonymity can be ensured. Instant messaging has been suggested to be beneficial and fitting to study sensitive research questions, and produces comparable results as face-to-face interviews (Ayling \& Mewse, 2009; Opdenakker, 2006). Two interviews were carried out in English and 28 in Dutch. All participants provided informed consent at the start of the interview.

We developed an interview guide with fifteen questions, related to the topics of PrEP use, PrEP-related healthcare, PrEP communication, and sexual risk behavior. Every question was copied (one by one) into the chat window from the interview guide. Interviews were held in a conversational style and additional questions were asked for clarification or elaboration. The order of questions was adapted to the flow of the conversation. The interviews took a maximum of two hours and were conducted uninterruptedly.

Twenty-six interviews were conducted by a female interviewer (RK, 23 years old, master student in health and social psychology) and the remaining four interviews were conducted by a male interviewer (MvD, 28 years old, PhD student in applied social psychology). The first two interviews were jointly conducted by the interviewers, to align the use of the interview guide, and to ensure consistency and quality of the subsequent interviews. 


\section{Data analysis}

The experiences of informal PrEP users were explored using interpretive description (Thorne, 2016). The data was sorted into categories in line with the topics of the interview guide, and we identified and described issues that emerged during the interviews. The interviews were coded in a deductive and iterative process, using the qualitative data analysis software ATLAS. ti version 8.3.0 (ATLAS.ti Scientific Software Development GmbH, 2018). The first five interviews were coded by the two researchers independently. The researchers discussed the coding to improve reliability of their interpretation. Inconsistencies in coding were resolved through discussion. Thereafter, the remaining interviews were coded by only one of two researchers ( 26 by RK and 2 by MvD). After coding, the results were saved in a summarized manner in an Excel table for further analysis. Quotes that were originally in Dutch were translated verbatim into English, when reported in the results section.

\section{Results}

\section{Participant characteristics and PrEP use}

The age of the participants ranged from 24 to 66 years; about half of the participants (40.0\%) were younger than 40 years. The participants resided all over the Netherlands: Most of the participants lived in an urban area (12 in Amsterdam, 11 in other cities in the Netherlands), and seven participants lived in a rural area of the Netherlands. Eighteen participants (60.0\%) were single and 12 participants (40.0\%) were in an open relationship. Nineteen participants (63.3\%) used PrEP in a daily regimen and 11 participants (36.7\%) used PrEP on demand. The average time since commencement of PrEP use was 15 months (range: 3 - 60 months).

\section{Methods of PrEP procurement}

Most participants obtained PrEP abroad, notably in Thailand, India, and South-Africa, where PrEP at the time $(2015$ - 2017) was available for a lower price than in the Netherlands (approximately $€ 30$ per 30 pills). They travelled to these countries for their jobs or for holidays, or they asked friends to bring PrEP from these countries. A minority also bought PrEP online from sources abroad, although some MSM experienced that their shipment was intercepted by customs in the Netherlands, or that the shipment took long to arrive. As soon as cheaper generic versions of PrEP became available in the Netherlands (November 2017), most participants switched to obtaining PrEP via local pharmacies, as it was faster and cheaper than buying PrEP abroad. However, some participants continued to obtain PrEP from sources abroad, as they reported to be used to the procedure, or found it more convenient since a 
friend obtained it for them, as illustrated by one participant:

“...because I am used to this way [of obtaining PrEP], and I don't have to do much effort for it, thus simply out of convenience." (P21)

\section{Challenges in access to PrEP-related healthcare services}

In the Netherlands, the healthcare services related to PrEP can be accessed via general practitioners (GP), medical specialists, pharmacies, and the public health centers for HIV/STI testing. Almost all participants received a prescription from their GP, which allowed them to buy PrEP in a pharmacy abroad or online, and later also in the Netherlands. Most of them were satisfied with their GP regarding PrEP care. Some participants perceived a lack of knowledge among their GPs about the use and effectiveness of PrEP, or even a lack of awareness. Nine of these participants indicated that their GP took time to learn about PrEP, for example by consulting a specialist in the hospital or a public health center. Moreover, participants themselves could be a source of information for their GPs, and provided PrEP information to their GPs:

"We [my partner and I] have a very open dialogue with our GP. He knows that we have an open relationship and he has always supported us in the use of PrEP. In fact, I am his informant when it comes to the latest information. He applauded that we take responsibility ourselves regarding safe sex." (P4)

It is noteworthy that some participants had selected their GP based on the gay friendliness of the GP, sometimes already before obtaining PrEP, which made it easier to discuss PrEP with their GP. At least three participants switched GPs when they wanted to obtain a PrEP prescription, as their previous GP did not want to support their needs:

"I had to switch GPs, as the previous one had very old-fashioned ideas about sex and numbers of partners. The one I have now is understanding, thinks along, thinks about alternatives, and in case of STIs he asks questions in a professional way." (P3)

After receiving the prescription from the GP, people can obtain PrEP in their pharmacy (note that in the Netherlands, clients are usually registered with a specific pharmacy). Six participants stated that their pharmacy had sufficient information about PrEP, whereas others stated that their pharmacy did not know a lot about PrEP. For example, the pharmacist assumed that they were HIV-positive and mistook PrEP for antiretroviral treatment (ART), or mistook PrEP for post-exposure prophylaxis (PEP). In one case made known to us, this lack of 


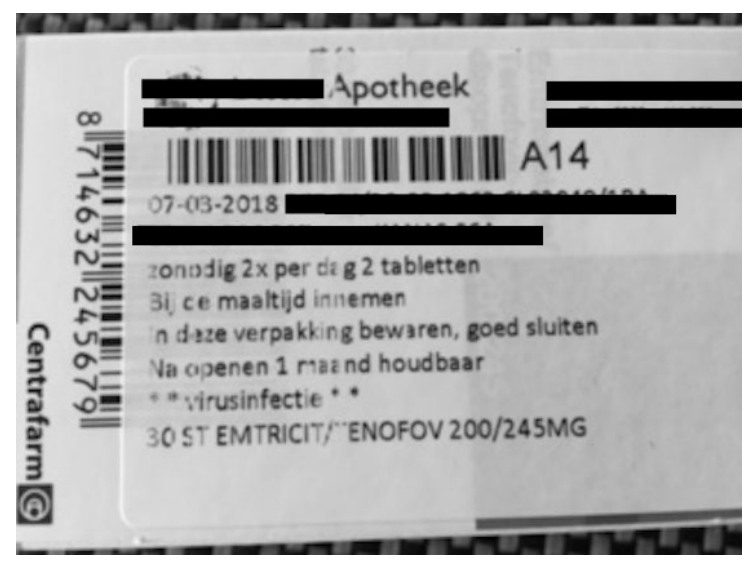

Figure 3.1. Image provided by an interview participant. Label on the package of generic PrEP pills of one of the participants, stating the misinformation regarding dosing scheme and the use for the treatment of a virus infection. Translated from Dutch: "Date: 07-03-2018. If necessary, 2 times per day 2 pills. Take with meals. Store in this package, close well. After opening one month shelf-life. ${ }^{\star *}$ virus infection ${ }^{\star * *}$. Please note that the correct PrEP dosing scheme is one pill per day for a daily regimen, and that PrEP is meant for the prevention instead of treatment of a virus infection.

knowledge amongst pharmacists resulted in putting misinformation and unclear information on the PrEP package, including an incorrect dosing scheme, and only mentioning "virus infection" without any reference to prevention (Figure 3.1). This puts PrEP users at risk of wrong dosage, and may lead to the misperception that the user is HIV positive, which could create social barriers to use PrEP:

“[The label on] the jar always states *virus infection*. I do not like that, because I just place it at home on the kitchen table. If you have people visiting, they immediately think you are sick." (P31)

This unclear, and sometimes erroneous, information about PrEP use from healthcare providers puts informal PrEP users at risk for incorrect PrEP use. It places a burden on PrEP users to be well informed about PrEP themselves to be able to educate their healthcare provider.

After obtaining PrEP, informal PrEP users can arrange regular HIV and STI testing at the public health centers (for free) or via the GP. Renal function testing can be done via the GP, but must be paid for by the client up to a maximum of $€ 385$ per year. Almost all participants 
reported that they underwent HIV and STI testing every three months. Renal function testing was done less frequent and by less participants compared to HIV and STI testing. A few participants did not do renal function testing at all, because they thought it was not necessary as they were using PrEP on demand, or because it was too expensive. This lack of renal testing demonstrates that while informal PrEP users often have a prescription for PrEP, medical supervision is not always present. Most participants had found a testing routine that worked well for them, with (free) STI testing carried out in public health centers and (paid) renal function testing done via their GP, although some participants reported difficulties with finding test locations for renal testing:

"[I do] not [test my renal function]. But I use [PrEP] sporadically, and your renal function should recover when you stop using the medication. I would like to test [renal function] more often, but I do not know how. At the STI clinic, where I come twice per year, they do not offer that." (P20)

Some participants also reported difficulties with making an appointment in public health centers as they faced waiting lists of more than four months for HIV and STI testing. Participants who lived in multiple places also mentioned that PrEP-related healthcare is more developed in Amsterdam compared to other places in the Netherlands:

"In the big cities it works. The rural area and the villages... for that matter the PrEP care is dependent on where you live, and that is worrisome." (P1)

To summarize, themes in challenges of informal users arranging their own access to PrEP-related healthcare services were gay-friendliness of GPs, lack of information and knowledge among GPs and pharmacists, and availability and pricing of testing facilities for HIV and STI testing as well as renal function testing.

\section{Community responses: PrEP related reactions from sex partners and friends}

Some participants did not disclose their PrEP use to (potential) sex partners, because they did not find it necessary to discuss this with the partner, as they saw PrEP as a preventative measure for themselves, independent of the other person. When participants disclosed their PrEP use, about half of them experienced negative reactions, especially in online dating apps. For example, they got rejected for a sex date, or they were seen as more promiscuous. On the other hand, most of the time participants experienced neutral or positive reactions by other MSM. For example, they were seen as taking care of their health. Moreover, informal PrEP 
users received questions on dating apps from other MSM who were interested in PrEP. They received questions about how to obtain PrEP and where to buy it.

Participants mentioned that other MSM assumed that PrEP users only want condomless sex, which was seen as both favorably and unfavorably by sex partners: Favorably because the other MSM preferred to have condomless sex and believed a PrEP user would agree on this; unfavorably because the other MSM associated PrEP use and condomless sex with irresponsibility:

"There are people who did not want to have sex with me, because I used PrEP and thus I was seen as irresponsible. This happens mainly with younger guys (16 - 24) and it happens approximately once every two months. I try to let these young guys realize that they also have a chance to get HIV, and this chance is much higher [when they have sex] with someone who does not use PrEP than with someone who uses PrEP." (P12)

When participants encountered negative reactions from other MSM on online dating apps, they noticed that this was sometimes a reflection of a lack of knowledge about PrEP. This was especially the case for participants from more rural areas or in the "early years" of PrEP use (2014-2015). As a result, PrEP users often found themselves educating other MSM about PrEP:

"Occasionally [I get] a negative reaction. But that is because they do not understand what it is and what it does. Then I explain it properly. [...] It arises often from ignorance, they think for example that you have HIV. Or they form a notion of that you are a dirty slut." (P14)

Some participants told their gay friends about their PrEP use and received mainly positive reactions. One participant mentioned that PrEP helped to raise issues of HIV and STI with gay friends. In addition, some participants mentioned that gay friends started PrEP use after discussing PrEP and sexual health:

"And also with gay friends, I have had more conversations about STI / HIV in the past six months than ever before. It feels really like a kind of smokescreen has disappeared. More and more [friends] use it. At least among my friends there is a lot of discussion about [PrEP]." (P30) 
The fact that PrEP was obtained informally did not stop others to want starting to use PrEP too. It helped informal PrEP users to connect socially, and one participant mentioned that he ordered PrEP in Thailand for his friends too.

Some participants also told their family and heterosexual friends about their PrEP use. Family and friends were surprised that something like PrEP existed, and were proud or jealous of how participants managed their sex life. The family of one participant was worried about his health and if PrEP (without condoms) would be enough protection for him. At least two participants did not tell their family about PrEP because they were ashamed to tell or feared to be judged.

\section{Changes in sexual behavior and sexual well-being}

Despite the informal way that these participants obtain their PrEP, they experienced similar responses to using PrEP as those reported by formal PrEP users. Most participants $(\mathrm{N}=22)$ reported a decrease in condom use since they started using PrEP. For some it was a deliberate decision to stop or decrease condom use when on PrEP, because they had erection problems when using condoms or they doubted the effectiveness of condoms. For others, condom use decreased because they felt less discipline to use condoms consistently. Some participants emphasized that it would not make sense for them to use both condoms and PrEP:

"During a PrEP information meeting two years ago, it was said that it is the intention that you use PrEP and condoms together. I thought that was nonsense at the time. Why use heavy medication if you still have to use a condom?" (P20)

Although there was a decrease in condom use for most PrEP users, there were situations in which most participants continued to use condoms. This was the case when the sex partner requested to use condoms, or when the participants assessed the sexual encounter as more risky. They evaluated this risk based on the level of familiarity with the sex partner, his PrEP use, and his assumed number of other sex partners:

"With a stranger I always do it safely, so with a condom. With a number of fuckbuddies I have unprotected sex. These are people who also use PrEP and I know that they do not have many changing contacts." (P4)

Changes in sexual behavior were related to improvements in sexual well-being. Most participants enjoyed sex without condoms more, and it contributed to a perceived increase in the 
quality of their sex life. Sex felt more unrestrained and intimate, and they had less worries during sex:

"For me a lot has changed [with anal intercourse]. It has mainly to do with condom use. [With condoms] it feels literally like there is no contact. When I am bottom, it is more painful. When I am top, I awfully quickly ejaculate. And a condom feels very unpleasant." - Interviewer: "So the quality of your sex life improved enormously?" P7: “Enormous." (P7)

The increased quality of their sex life was also reflected in changes in sexual experience. Participants felt less worried about getting HIV, and this resulted in increases in sexual activity and less concerns about who to have sex with. They also reported being able to enact fantasies that they did not dare to before using PrEP. For example, some participants reported that they felt more comfortable now with being the receptive partner (bottom) during anal intercourse:

"I also notice that I now dare to give in to fantasies that I have always been cautious about. [...] I also have sex with more men now. [...] Now I can do things that I like. Before, I found it scarier to be fucked in the passive role. Even with a condom. Does it slide off, or would someone take it off without a word? I was more preoccupied with these control thoughts, while one wants to indulge in sex. These thoughts restrained me in experiencing sex spontaneously." (P5)

For some participants the use of PrEP felt liberating, mostly because they felt less fear of HIV. About half of the participants reported memories of the early days AIDS, and some of them reported that they always associated sex with fear of HIV, which limited their sexual pleasure:

"Every morning after having sex with a guy, I start thinking about whether there has been a risk of HIV. So instead of feeling comfortable [with having sex with a guy], the fear dominates. Since I take PrEP, the fear has been gone. [...] Gay sex and HIV are almost directly related to each other. [...] Instead of sex being something pleasant, it is something dangerous for gays because one can run into a serious problem." (P17)

Also younger participants, who became sexually active in a time when HIV was a manageable condition, reported fear of HIV, which was reduced by the use of PrEP:

"Before I used PrEP, I sometimes could not sleep well, and I regretted often having sex. Also I could not really enjoy sex fully. I was aware that I would ever get HIV, the only 
question was when.” (P12)

For others, condomless sex itself felt liberating. After many years of using condoms, they felt hesitation about not using condoms, but they got easily accustomed to it:

"It took me some effort to accept the idea that sex without a condom was also "safe" for HIV. But after having bare sex with my regular sex partner, it was such a liberation that I was soon over it." (P28)

About half of the participants reported using recreational drugs during sex. Most of them already used drugs before they started using PrEP. For some participants drug use increased, as it often co-occurred with going to sex parties. PrEP was beneficial for them as they had to worry less about condomless sex:

"I mostly use XTC [ecstasy/MDMA] and GHB [when going to sex parties]. I do not use it always, but when I do, the use of PrEP is nice, because you do not always know afterwards what happened. [The use of PrEP and drugs] are independent of each other. But the use of PrEP has many advantages, I think. I do not have to worry about how the sex is being done." (P6)

\section{Discussion}

The aim of this study was to explore the experiences of informal PrEP users in a context where PrEP is formally available, but formal implementation is still limited. We in particular explored the experiences of informal PrEP users across three domains: Access to PrEP and PrEP-related healthcare, community responses, and sexual behavior and well-being.

\section{Access to PrEP and PrEP-related healthcare}

We found that informal PrEP users have found their own ways and routines in accessing PrEP and PrEP-related healthcare. Many of our participants switched from obtaining PrEP abroad to obtaining PrEP via local pharmacies in the Netherlands, as soon as generic PrEP became more affordable in the Netherlands. In contrast with earlier studies that found that informal PrEP users are less likely to undergo frequent HIV and STI testing (AIDES, 2016; Buttram, 2018; Dimitrov et al., 2013), most participants in our interviews tested regularly. While this paints a positive picture of the uptake of PrEP healthcare services among informal PrEP users, findings also identify several major concerns. 
Firstly, GPs, who play a critical role as gatekeepers in the Dutch healthcare system, were not always able or willing to support the needs of informal PrEP users. The participants in our study were well-informed about PrEP, and that helped them to either educate their GPs, or to change to another GP that did support their needs. MSM who are less informed about PrEP may however not be able to get the support they need from their GP, which would preclude them from accessing PrEP, or could force them to obtain PrEP via informal channels.

Secondly, some participants experienced long waiting lists for HIV and STI testing at public health clinics. While this not only puts PrEP users at risk of having and transmitting an undetected STI, it may also result in them doing less testing, not testing, or discontinuing PrEP use (Whitfield et al., 2018), which eventually may increase their risk of HIV.

Thirdly, renal function testing was not always optimal, as was also found before among informal PrEP users in Germany (Koppe et al., 2019). Similar to the "DIY PrEP" study (Paparini et al., 2018), we found that some PrEP users find renal function testing not always necessary, but also that some PrEP users did not know where to go for these tests, as it was not available in the public health clinics where HIV and STI testing are offered. In addition to setting up adequate PrEP consultations to inform MSM about the use and potential side effects of PrEP, a convenient testing location should be organized where informal and formal PrEP users can go for all the necessary tests on a regular basis.

\section{Community responses}

PrEP users mostly got positive reactions. For example, they were seen as more desirable sex partners. It has been suggested that PrEP users are more likely to be seen as HIV-negative, and therefore have a lower chance to transmit HIV, making them a more desirable sex partner (Martinez \& Jonas, 2019; Medina et al., 2019). While media outlets can present PrEP use in a stigmatizing way (i.e. "Truvada whores" (Calabrese \& Underhill, 2015; Schwartz \& Grimm, 2017)), and stigmatizing reactions even occur among PrEP users themselves (Puppo et al., 2020), our study provides a more nuanced view on how informal PrEP users are seen by community members in which general PrEP knowledge is emerging.

PrEP users nevertheless encountered negative, or stigmatizing, reactions. This included being rejected by a potential sex partner, or receiving negative comments in dating apps. Negative responses were found to be especially common in the early years of PrEP availability in the Netherlands and in rural areas, suggesting that unfamiliarity with PrEP may lead to negative responses. The informal PrEP users in this study were able to deal with negative reactions, and 
were able to educate other MSM about their PrEP use, because they were well-informed. Especially in countries where PrEP is not formally implemented, MSM are more likely to be informed about PrEP via peers, and they may find it challenging to obtain reliable information.

\section{Sexual behavior and well-being}

Most informal PrEP users reported an increased quality of their sex life, mainly driven by less fear for HIV infection and decreased condom use. Although it is not surprising that condom used decreased, and this has been found before (Traeger et al., 2018), it is an important finding that condom use was a prominent topic in many of the interviews. It often played a role in participants' considerations on how PrEP increased the quality of their sex life, as PrEP use helped participants to explore ways of having sex, for example by switching sexual positions (insertive/receptive) or by experimenting with substance use and participating in sex parties. This is in line with findings from other countries and in earlier studies (Collins et al., 2017; Hojilla et al., 2016; Mabire et al., 2019; Paparini et al., 2018), and seems to reflect an overall trend, regardless of whether the type of procurement and use is informal or formal. Participants noted that the reduced fear for HIV felt liberating and improved their sex life. The sexual liberation related to PrEP use has been found before (Collins et al., 2017; Grace et al., 2018), and indicates that PrEP users feel more in control of their sex life (Philpot et al., 2020).

\section{Continuing informal PrEP use or moving towards formal PrEP}

In our study we found that many PrEP users switched from the informal procurement of PrEP to a formal procurement of PrEP in local pharmacies. At the same time, PrEP implementation programs were not in place yet, so PrEP-related healthcare, in particular counseling, and regular HIV, STI and renal function testing was not formally accessible for them. The participants in our study had to organize this themselves, and most were quite well able to do so.

Informal PrEP procurement may continue to exist, even when the formal accessibility of PrEP is improved. On a personal level, MSM might prefer to continue obtaining PrEP via informal channels, as one participant mentioned in our study that he did this out of convenience. A study in Germany found that, despite the formal availability of PrEP, $17.4 \%$ of PrEP users continued to obtain PrEP informally, mainly because the price of generic PrEP ( $€ 50$ per month) was still unaffordable to them (Koppe et al., 2019). On a structural level, there are many countries in the world where no steps are taken (yet) to make PrEP available (see www.prepwatch. org for country updates, and for Europe the ECDC report (European Centre for Disease Prevention and Control, 2019c)), so for many (potential) PrEP users around the world there are 
no opportunities yet to formally access PrEP use.

We found that even when participants switched to formal PrEP procurement, formal PrEP-related healthcare was not always readily accessible. To facilitate a transition into formal PrEP-related healthcare, it is important to consider the needs of PrEP users. The PrEP-related healthcare service should provide one-stop access to counseling, renal function testing, and HIV and STI testing. The service should not only be affordable, but also convenient and non-judgmental. There are already several initiatives to offer these services to informal PrEP users, such as the InPrEP project in Amsterdam (GGD Amsterdam, 2017) and CheckpointLX in Lisbon, Portugal (Ribeiro \& Rocha, 2019).

\section{Limitations}

Several limitations of this study should be noted. We recruited participants via a survey that was posted on the advocacy website PrEPnu.nl, which provides information on accessing and using PrEP. This means that our participants had access to extensive information on PrEP. It is also important to note that the informal PrEP users in this study can be considered innovators or early adopters of PrEP, who had to be well-informed in order to be able to obtain PrEP. Their knowledge also allowed some of them to convince their GP to prescribe PrEP, and to arrange regular HIV and STI testing themselves. The recruitment and sample characteristics may limit the generalizability of the results of our study.

Furthermore, we used online chat messaging to conduct the interviews. While this may lead to less detailed responses (Davis et al., 2004), the results are overall comparable to those of studies based on face-to-face interviews (Ayling \& Mewse, 2009; Opdenakker, 2006). The interviewers were specifically instructed to invite participants to elaborate, in particular regarding their emotions or feelings. Nevertheless, some participants kept their answers rather short. We believe that online chatting is a valuable data collection tool, as it facilitates anonymous interaction. Moreover, in the context of e-health interventions it is not uncommon for MSM to discuss PrEP and sexual behavior online (Schnall et al., 2014). This makes the transition to discuss these topics in an online chat interview relatively easy.

\section{Conclusions}

This study highlighted the experiences and behaviors of informal PrEP users in a context of transition to full formal PrEP healthcare implementation. Overall, informal PrEP users 
were doing quite well regarding PrEP use and accessing PrEP-related healthcare. PrEP healthcare services should not only be accessible to formal PrEP users, but also to PrEP users who procure PrEP informally. Global and national guidelines may be helpful to implement these services, but as the example of the Netherlands in this study shows, the availability of national guidelines alone does not directly make practice. Informal PrEP users still have to take the initiative to inform their healthcare providers about their self-medication, which may be frowned upon, and bears the risk of not receiving the necessary care due to avoiding disclosure of PrEP use. The experiences of informal PrEP users regarding community responses and sexual behavior were in line with findings of studies of PrEP use in a formal context. The findings of our study are not only relevant for the Netherlands, but also for other high-income countries where formal PrEP is not implemented yet. 


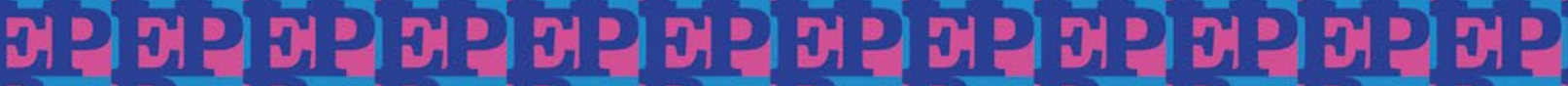

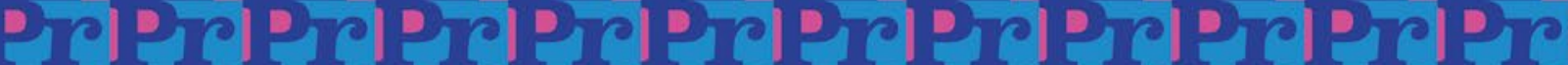
A

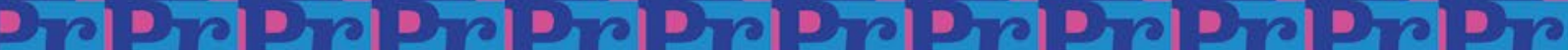
A

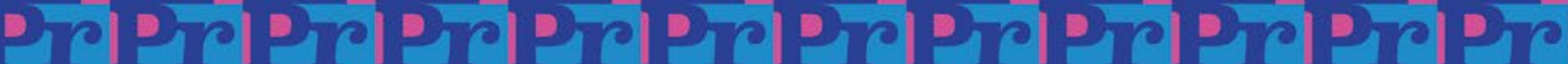

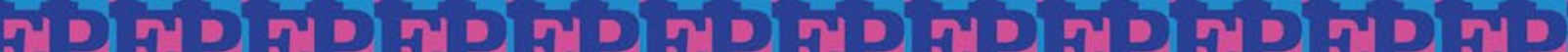
dו

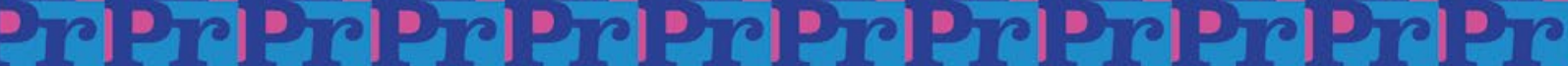
A

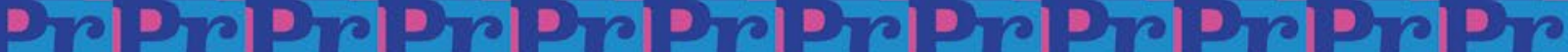

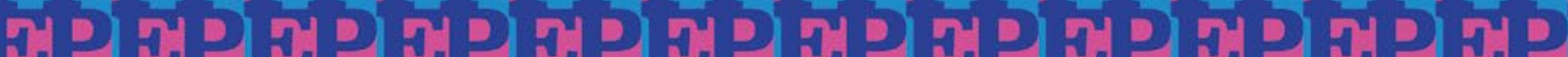
ת

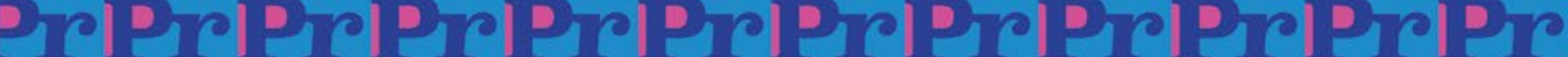

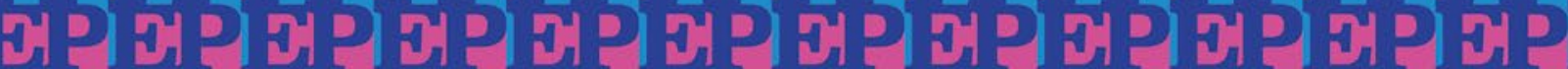

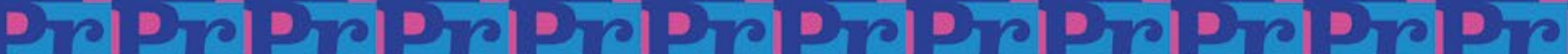
A

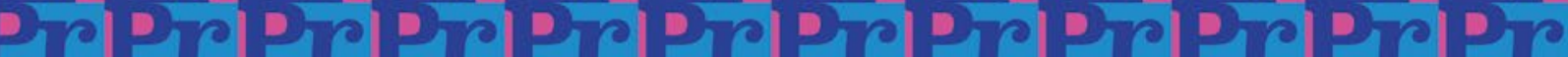

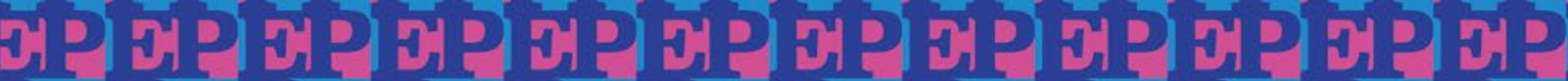

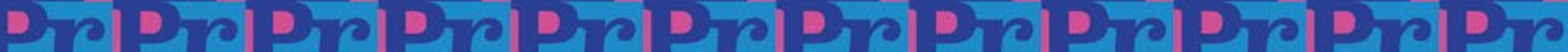

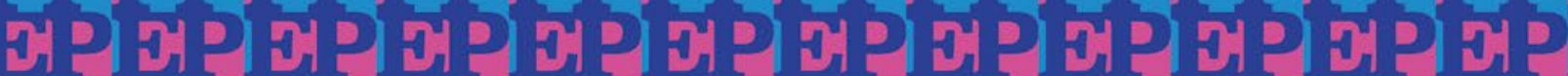

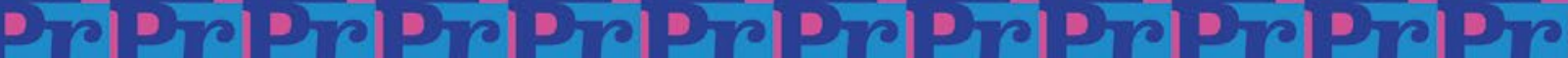
A

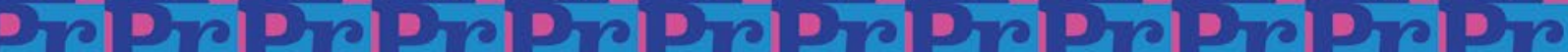
A

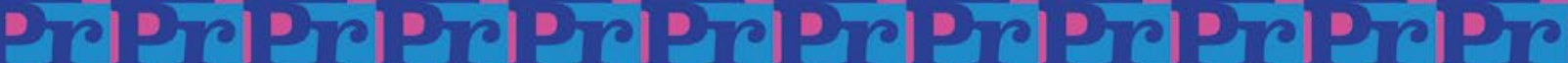

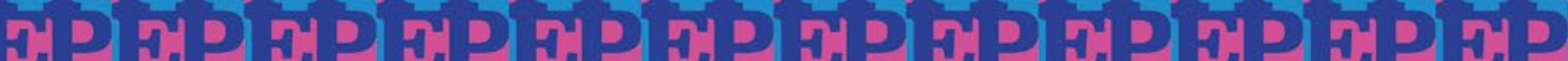

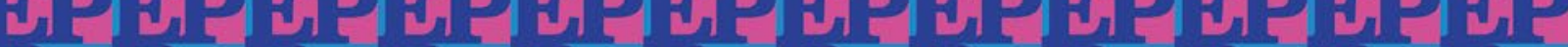

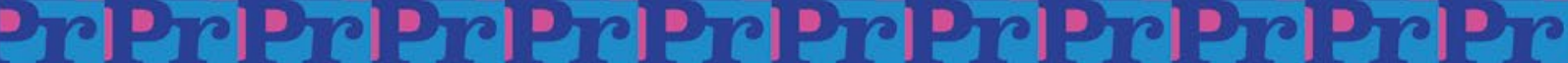
A

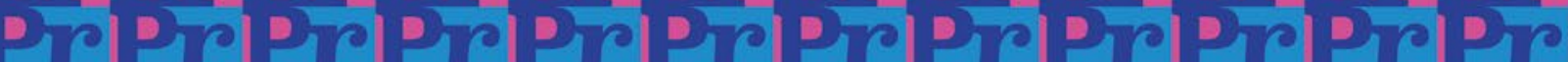
प II

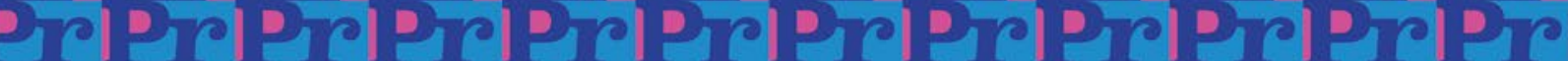
$\mathrm{D}$
$\mathrm{A}$

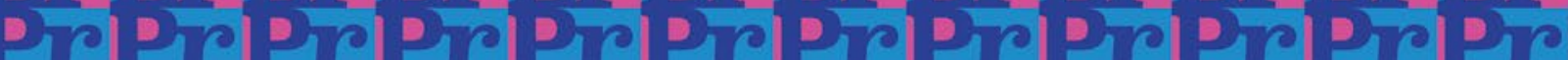




\section{CHAPTER 4}

\section{Slow uptake of PrEP: Behavioral predictors and the influence of price on PrEP uptake among MSM with a high interest in PrEP}

This chapter is based on:

Van Dijk, M., De Wit, J.B.F., Guadamuz, T.E., Martinez, J.E., \& Jonas, K.J. (2021). Slow uptake of PrEP: Behavioral predictors and the influence of price on PrEP uptake among MSM with a high interest in PrEP. AIDS and Behavior. https://doi.org/10.1007/s10461-021-03200-4 


\begin{abstract}
Despite the improved availability and affordability of PrEP in the Netherlands, PrEP uptake is low among men who have sex with men (MSM). To optimize uptake, it is important to identify facilitators and barriers of PrEP use. During our study period, the price of PrEP dropped significantly after generic PrEP was introduced. We investigated whether the price drop predicts PrEP uptake, alongside behavioral and demographic characteristics. Participants $(N=$ 349) were recruited online and completed three questionnaires over a period of six months, between February 2017 and March 2019. After six months, 159 (45.6\%) participants were using PrEP. PrEP uptake was greater among MSM who ever had postexposure prophylaxis (PEP) treatment, among MSM with a better perceived financial situation, and when the price of PrEP dropped. MSM in a tighter perceived financial situation may use PrEP more when it would be free or fully reimbursed.
\end{abstract}




\section{Introduction}

Pre-Exposure Prophylaxis (PrEP) has been found to be an effective biomedical intervention for HIV prevention (Fonner et al., 2016; Grant et al., 2010, 2014; J. M. Molina et al., 2017). The World Health Organization (WHO) recommends PrEP for people who are at substantial risk of HIV infection, for example men who have sex with men (MSM) (World Health Organization, 2015). In the past years, efforts have been made to make PrEP accessible, for example by setting up national PrEP implementation guidelines (CDC, 2017; Hoornenborg $\&$ Rijnders, 2016). Despite its effectiveness and these efforts, the accessibility of PrEP varies greatly per country (AVAC, 2020), the uptake of PrEP has been low (Parsons et al., 2017), and the full potential of PrEP at population level has not been reached yet (Pyra et al., 2019). In the Netherlands, it is estimated that there are currently 3,500 individuals on PrEP (AVAC, 2020), of which 95\% are MSM (Staritsky et al., 2020), while it is estimated that 10,000 MSM meet eligibility criteria for PrEP (Hoornenborg, Krakower, et al., 2017; Reitsema et al., 2020). To increase uptake, it is therefore important to identify facilitators and barriers of PrEP use. While earlier studies investigated behavioral and psychological factors of PrEP uptake, such as sexual risk behaviors, perceived HIV risk, and stigma (Blumenthal et al., 2019; Dubov, Altice, et al., 2018; Golub, Fikslin, Goldberg, et al., 2019; Haire, 2015) , the influence of actual price changes on PrEP use has not been fully investigated, especially not in contexts where universal health care coverage of PrEP is not (yet) available.

Previous studies found several behavioral factors to be related to PrEP use. Compared to MSM who were not using PrEP, PrEP users were more likely to have had a recent STI diagnosis, have used post-exposure prophylaxis (PEP) before, have had condomless anal intercourse, have used recreational drugs or practiced chemsex (i.e., use of certain stimulants in the context of sex), have had sex with HIV positive sex partners, and have a greater number of sex partners (Bourne et al., 2019; Eaton et al., 2018; Holloway et al., 2017; Okafor et al., 2017). In terms of demographic characteristics, PrEP users were found to be of middle age and to have a higher income (Bourne et al., 2019; Holloway et al., 2017). While it is reassuring to see that MSM at higher risk of HIV are more likely to be interested in PrEP and to use PrEP, overall PrEP remains "underused", and some population strata at higher risk are less likely to use PrEP (Kuhns et al., 2017; Marcus et al., 2016; Shover et al., 2018).

The slow uptake of PrEP so far has been explained by structural and psychosocial barriers, including lack of access to PrEP, doubts about effectiveness, concerns about side-effects, and ex- 


\section{Chapter 4}

pected stigma (Calabrese \& Underhill, 2015; Golub et al., 2013; Hannaford et al., 2018; Pinto et al., 2018; Thomann et al., 2017). Moreover, the costs of PrEP have been noted as one of the main barriers for PrEP uptake in cross-sectional analysis (Arnold et al., 2017; Bauermeister et al., 2014; Dubov et al., 2019; Goparaju et al., 2017; Kubicek et al., 2015; Pérez-Figueroa et al., 2015; Rice et al., 2019; Schwartz \& Grimm, 2017). Notably, in a report drawing on data from 32 European and Asian countries, the price of PrEP was the most common barrier for PrEP uptake (Hayes et al., 2019). With prices of around $€ 500$ per month for patented Tenofovir-Emtricitabine formulations in Europe and \$ 1600 per month in the U.S., branded PrEP is likely unaffordable for most people in many countries.

In recent years, PrEP has however become more affordable and accessible as a result of the introduction of generic formulations of PrEP and the inclusion of PrEP in health care packages or insurance coverage (AVAC, 2020; PrEPnu, 2017). PrEP uptake may increase as it becomes more affordable, and this hypothesis is further supported by the finding that uninsured MSM are less likely to use PrEP (Kuhns et al., 2017; Patel et al., 2017), if PrEP is included in health insurance coverage. Yet, even though the affordability of PrEP is increasing, current pricing may still be a barrier for certain individuals and groups, notably MSM with lower incomes.

In the current study we examine whether the costs of PrEP indeed predict PrEP uptake, alongside behavioral and demographic characteristics. As of 1 January 2018 the price of PrEP in the Netherlands decreased from $€ 500$,- to $€ 50$,- per month, as a result of the introduction of generic formulations of PrEP (PrEPnu, 2017). This introduction of generic PrEP allowed us to look more closely into the effects of price on the uptake of PrEP. At the time of our study, PrEP was not included in reimbursement schemes of the national health insurance. The primary way of obtaining PrEP was to buy PrEP at the pharmacy on prescription from the general practitioner (Van Bergen, 2019). Formal PrEP services, offering PrEP in a co-payment scheme, were implemented in the public health centers as of July 2019, after data collection of our study was finished (Ministry of Health Welfare and Sport, 2019; Staritsky et al., 2020).

\section{Methods}

\section{Participants and procedure}

Participants were recruited via PrEPnu.nl, the website of the Dutch PrEP advocacy group PrEPnu (Dutch for PrEPnow), between February 2017 and March 2019. Every consenting participant in the baseline survey (T0) received follow-up questionnaires via email after three 
(T1) and six months (T2). Participants who did not complete the T1 questionnaire were still encouraged to complete the T2 questionnaire. Participants younger than 18 years old or living with HIV were excluded from participation. All participants who completed at least two questionnaires $(\mathrm{T} 0+\mathrm{T} 1 / \mathrm{T} 2)$ could enter into a raffle to win a $€ 100$,- gift card. The Ethics Review Committee Psychology and Neuroscience of Maastricht University approved this study (ERCPN-174_10_12_2016). In the current study, we did not use the data from the T1 questionnaire, as this resulted in a smaller sample size because some T0 and T2 participants did not complete the $\mathrm{T} 1$ questionnaire.

In total, 766 participants completed the baseline (T0) questionnaire. For the current analysis, we only included MSM who were not using PrEP at baseline, and completed the items in the T2 questionnaire that are pertinent for the current analyses. This resulted in a sample size of $N=349$.

\section{Measures}

Given the lack of published or validated instruments at the onset of the study, questionnaire items were drawn from the earlier Flash PrEP in Europe study (AIDES, 2016), or newly designed by the researchers. Questionnaires were administered online using Qualtrics.com; participants could not revert back to previous questions. The questionnaire was offered in Dutch and English. The full questionnaire can be found on https://osf.io/dm79v/. Below we describe the relevant variables for the current analyses.

\section{Sociodemographic characteristics}

In the first questionnaire (T0) participants were asked to indicate their gender, age, relationship status, educational level, financial situation, country of birth, and country of residence. Gender was determined using two questions: gender assigned at birth and current gender. Educational level was indicated by five levels, ranging from no tertiary education to $\mathrm{PhD}$ degree. Financial situation was assessed with a 6-point scale: (1) 'you can't make ends meet without borrowing', (2) 'you are having problems making ends meet', (3) 'you are getting by but have to be careful', (4) 'things are all right', (5) 'you are doing rather well', and (6) 'you are doing really well. The sociodemographic items were not repeated at T1 or T2, as we considered these characteristics to be stable over a period of 6 months. Relationship status was again asked at $\mathrm{T} 2$, as this is more likely to change over time.

\section{PrEP related items}

At T2, we asked whether participants were taking PrEP, using a question with sex response 


\section{Chapter 4}

options (Yes, I use PrEP daily / Yes, I use PrEP intermittently (more or less every time I have sex) / Yes, I use PrEP recreationally (on demand; during special phases/moments when I have sex) / No, but I have used PrEP before (less than six months ago) / No, but I have PrEP used before (more than six months ago) / No, I haven't used PrEP at all). We decided to distinguish between "intermittent" and "recreational" PrEP to capture a possible difference between MSM who use PrEP more frequently (but not daily) and others who use PrEP less systematically and more recreationally or season-based (Elsesser et al., 2016; Hojilla et al., 2016; Underhill et al., 2018). Since the development of our questionnaire (2017) there have been changes in terminology. Currently the common term for "intermittent PrEP" is "on demand PrEP". There is no consensus regarding the terminology to refer to more recreational or seasonal PrEP use, although it is noted "recreational" use may inadvertently imply non-prescribed PrEP use (Kuo et al., 2020). Participants who were using PrEP were asked to indicate how they obtained PrEP, with seven response options: via HIV positive friend(s), through PEP treatment, through a PrEP research trial, at a local pharmacy, via a buyers club, at pharmacies abroad, and at online pharmacies.

\section{Sexual risk behavior}

Participants were asked whether they used a condom the last time they had anal intercourse (yes / no). In addition, we asked whether participants had used drugs in a sexual context (yes / no). Participants were also asked to indicate the number of sex partners they had in the past six months.

\section{Sexual health}

Participants were asked whether they ever had PEP treatment (yes / no) and whether they ever had an STI (yes, in the past 12 months / yes, more than 12 months ago / no).

\section{PrEP pricing}

Price of PrEP was dummy coded as " 0 ", indicating that when the participant completed the T2 questionnaire only branded PrEP was available at pharmacies in the Netherlands at a cost of $€ 500$ (until 01-01-2018), or "1", indicating that when the participant completed the T2 questionnaire generic PrEP was available at pharmacies in the Netherlands, at a price of $€ 50$ or lower (after 01-01-2018). 


\section{Data analysis}

We analyzed the data using IBM SPSS Statistics version 26. We controlled for duplicate participation by participant identifiers. We used descriptive statistics to describe the sociodemographic characteristics of the sample. We analyzed only the data of participants who were not using PrEP at T0 $(\mathrm{N}=344)$, to investigate the factors at baseline (T0) potentially related to PrEP initiation after six months (T2), using multivariate logistic regression analysis. The following independent variables were included in the model: age, number of sex partners in the past six months, educational level, perceived financial situation, relationship status, STI history, having used a condom at last anal intercourse, having used drugs in a sexual context, and having ever had PEP treatment. Additionally, we added the variable 'price of PrEP' (at T2) to the model to investigate the influence of the price of PrEP on PrEP initiation. We conducted a post-hoc interaction analysis of the effect of the price of PrEP on PrEP initiation, stratified by different levels of the perceived financial situation (Ai \& Norton, 2003; Hayes \& Matthes, 2009).

\section{Results}

\section{Participant characteristics}

Descriptive characteristics of the sample are shown in Table 4.1. Most participants identified their gender as male (344; 98.6\%), and the remaining five identified as non-binary or preferred not to answer. The average age of participants was 41 years (range: 18 - 75). About half of participants were single (196; 56.2\%), 136 (39.0\%) were in an open relationship, and 17 (4.9\%) were in a monogamous relationship. More than half of the participants had a Bachelor degree or higher $(218 ; 62.4 \%)$, and on average they perceived their financial situation as quite favorable $(M=4.35, S D=1.13$, median $=4)$.

At T2, 159 (45.6\%) participants were using PrEP or had ever used PrEP in the past. Almost half had used PrEP daily (75; 47.2\%), 40 used PrEP on demand (25.2\%), 32 used PrEP recreationally (20.1\%), and 12 had (temporarily) stopped taking PrEP (7.5\%). Most participants obtained PrEP via a doctor's prescription and paid for PrEP themselves at a pharmacy in the Netherlands (104; 65.4\%), 31 (19.5\%) obtained PrEP from a pharmacy abroad, 10 (6.3\%) obtained PrEP through participation in a research trial, 9 (5.7\%) obtained PrEP via a buyer's club, 8 (5.0\%) obtained PrEP via online pharmacies abroad, 6 (3.8\%) obtained PrEP via PEP treatment, and 3 (1.9\%) obtained PrEP via HIV-positive friends. Some participants obtained PrEP via multiple channels. When stratified by financial situation, we found that participants 
Table 4.1. Participant characteristics.

\begin{tabular}{|c|c|c|c|}
\hline & $\begin{array}{l}\text { Total sample } \\
\quad N=349\end{array}$ & $\begin{array}{l}\text { PrEP users } \\
\quad n=159\end{array}$ & $\begin{array}{c}\text { Non-PrEP users } \\
\quad n=190\end{array}$ \\
\hline Age (years; $M$, range) & $41(18-75)$ & $42(20-66)$ & $40(18-75)$ \\
\hline \multicolumn{4}{|l|}{ Gender } \\
\hline Male & $344(98.6 \%)$ & $157(98.7 \%)$ & $187(98.4 \%)$ \\
\hline Non-binary / unknown & $5(1.4 \%)$ & $2(1.3 \%)$ & $3(1.6 \%)$ \\
\hline Born in the Netherlands & $281(80.5 \%)$ & $125(78.6 \%)$ & $156(82.1 \%)$ \\
\hline Living in the Netherlands & $335(96.0 \%)$ & $154(96.9 \%)$ & $181(95.3 \%)$ \\
\hline $\begin{array}{l}\text { Perceived financial situation }(M, S D ; \\
\text { scale } 1=\text { You can't make ends meet } \\
\text { without borrowing, to } 6=\text { You are doing } \\
\text { really well) }\end{array}$ & $4.35(1.13)$ & $4.62(1.02)$ & $4.12(1.16)$ \\
\hline \multicolumn{4}{|l|}{ Education level } \\
\hline Master and $\mathrm{PhD}$ & $101(28.9 \%)$ & $49(30.8 \%)$ & $52(27.4 \%)$ \\
\hline Bachelor & $117(33.5 \%)$ & $48(30.2 \%)$ & $69(36.3 \%)$ \\
\hline $\begin{array}{l}\text { High school and Professional } \\
\text { qualification }\end{array}$ & $131(37.5 \%)$ & $62(39.0 \%)$ & $69(36.3 \%)$ \\
\hline \multicolumn{4}{|l|}{ Relationship status } \\
\hline Single & $196(56.2 \%)$ & $86(54.1 \%)$ & $110(57.9 \%)$ \\
\hline In a relationship & $17(4.9 \%)$ & $5(3.1 \%)$ & $12(6.3 \%)$ \\
\hline In an open relationship & $136(39.0 \%)$ & $68(42.8 \%)$ & $68(35.8 \%)$ \\
\hline \multicolumn{4}{|l|}{ STI } \\
\hline No & $92(26.4 \%)$ & $38(23.9 \%)$ & $54(28.4 \%)$ \\
\hline Yes in the past 12 months & $116(33.2 \%)$ & $65(40.9 \%)$ & $51(26.8 \%)$ \\
\hline Yes more than 12 months ago & $141(40.4 \%)$ & $56(35.2 \%)$ & $85(44.7 \%)$ \\
\hline Used a condom the last time & $161(46.1 \%)$ & $76(47.8 \%)$ & $85(44.7 \%)$ \\
\hline Used drugs in a sexual context & $174(49.9 \%)$ & $85(53.5 \%)$ & $89(46.8 \%)$ \\
\hline Ever had a PEP treatment & $41(11.7 \%)$ & $24(15.1 \%)$ & $17(8.9 \%)$ \\
\hline $\begin{array}{l}\text { Number of sex partners in past } 6 \text { months } \\
(M, S D)\end{array}$ & $14.96(19.12)$ & $18.26(24.18)$ & $12.19(12.95)$ \\
\hline
\end{tabular}

who perceived their financial situation as "really well" were more likely to buy PrEP from pharmacies abroad compared to participants with a lower perceived financial situation (see Appendix A of this chapter). Participants who perceived their financial situation as "having to be careful about expenses" or lower were less likely to use PrEP at all. 
Table 4.2. Bivariate and multivariate logistic regression examining correlates of PrEP initiation.

\begin{tabular}{|c|c|c|c|c|}
\hline & \multicolumn{2}{|c|}{ Bivariate } & \multicolumn{2}{|c|}{ Multivariate } \\
\hline & OR & $95 \% \mathrm{CI}$ OR & $\mathrm{aOR}$ & $95 \% \mathrm{CI}$ aOR \\
\hline Age & 1.01 & $1.00-1.03$ & 1.01 & $0.99-1.03$ \\
\hline $\begin{array}{l}\text { Number of sex partners in past } \\
6 \text { months }\end{array}$ & $1.02^{\star *}$ & $1.01-1.04$ & 1.01 & $1.00-1.03$ \\
\hline Perceived financial situation & $1.52^{\star * *}$ & $1.24-1.86$ & $1.50^{\star \star \star}$ & $1.21-1.87$ \\
\hline \multicolumn{5}{|l|}{ Education level } \\
\hline Master and $\mathrm{PhD}$ & Ref & & Ref & \\
\hline Bachelor & 0.74 & $0.43-1.26$ & 0.97 & $0.54-1.74$ \\
\hline $\begin{array}{l}\text { High school and } \\
\text { Professional qualification }\end{array}$ & 0.95 & $0.57-1.60$ & 1.11 & $0.63-1.96$ \\
\hline \multicolumn{5}{|l|}{ Relationship status } \\
\hline Single & Ref & & Ref & \\
\hline In an open relationship & 1.28 & $0.83-1.98$ & 1.15 & $0.70-1.87$ \\
\hline In a relationship & 0.53 & $0.18-1.57$ & 0.46 & $0.14-1.51$ \\
\hline \multicolumn{5}{|l|}{ STI } \\
\hline Never had an STI & Ref & & Ref & \\
\hline $\begin{array}{l}\text { Had an STI in the past } 12 \\
\text { months }\end{array}$ & $1.81^{\star}$ & $1.04-3.15$ & 1.57 & $0.83-2.95$ \\
\hline $\begin{array}{l}\text { Had an STI more than } 12 \\
\text { months ago }\end{array}$ & 0.94 & $0.55-1.60$ & 0.72 & $0.40-1.31$ \\
\hline $\begin{array}{l}\text { Not used a condom the last } \\
\text { time }^{\mathrm{a}}\end{array}$ & 1.13 & $0.74-1.73$ & 1.17 & $0.72-1.88$ \\
\hline Used drugs in a sexual context $\mathrm{t}^{\mathrm{a}}$ & 1.30 & $0.86-1.99$ & 1.26 & $0.78-2.06$ \\
\hline Ever had a PEP treatment ${ }^{a}$ & 1.81 & $0.93-3.50$ & $2.34^{*}$ & $1.12-4.86$ \\
\hline Price of $\operatorname{PrEP}^{b}$ & $1.71^{*}$ & $1.03-2.84$ & $1.91^{*}$ & $1.09-3.32$ \\
\hline
\end{tabular}

a The reference category for these variables is "no".

b The variable 'Price of PrEP' was coded with 0 (when the participant completed the survey at the time when PrEP was $€ 500$ per month, before 01-01-2018) and 1 (when the participant completed the survey at the time when PrEP was $€ 50$ per month, after 01-01-2018).

${ }^{\star} p<.05,{ }^{* *} p<.01,{ }^{* *} p<.001$

$\chi^{2}(13, N=349)=46.08, p<.001$, Nagelkerke $R^{2}=.165$

\section{Predictors of PrEP initiation after six months}

The outcomes of the logistic regression analysis of correlates of PrEP initiation are shown in Table 4.2. Significant multivariable correlates of PrEP initiation included perceived financial situation $(\mathrm{aOR}=1.50,95 \% \mathrm{CI} 1.21-1.87)$, having ever had PEP treatment $(\mathrm{aOR}=2.34,95 \%$ CI 1.12 - 4.86), and the price of $\operatorname{PrEP}(\mathrm{aOR}=1.91,95 \%$ CI $1.09-3.32)$. 
Table 4.3. Examining the conditional effect of price of PrEP on PrEP use at different levels of perceived financial situation in the multivariate logistic regression.

\begin{tabular}{lcc}
\hline & aOR & $95 \%$ CI aOR \\
\hline One SD below mean financial situation (3.22) & 2.06 & $0.88-4.82$ \\
At mean financial situation (4.35) & $1.83^{\star}$ & $1.06-3.17$ \\
One SD above mean financial situation (5.47) & 1.62 & $0.75-3.50$ \\
\hline
\end{tabular}

${ }^{\star} p<.05$

In a post-hoc analysis we investigated the effect of the price of PrEP on PrEP initiation at three levels of perceived financial situation: The average level of perceived financial situation in the sample and at levels of perceived financial situation one standard deviation below as well as one standard deviation above the average perceived financial situation (Table 4.3). We found that the price of PrEP was only related to PrEP initiation when perceived financial situation was at an average level ( $\mathrm{aOR}=1.83,95 \%$ CI $1.06-3.17)$.

\section{Discussion}

The aim of this study was to investigate what sociodemographic and behavioral factors predict PrEP uptake. We in particular investigated whether the price decrease of PrEP, from $€ 500$,- to $€ 50$,- per month, resulting from the introduction of generic formulations of PrEP, is associated with an increased PrEP uptake. This study found that a better perceived financial situation, having ever had PEP treatment, and the price decrease of PrEP were significantly related to PrEP initiation. This is in line with our hypotheses and findings of earlier studies. However, while earlier cross-sectional studies reported the price of PrEP to be an important overall barrier for intended PrEP uptake (Arnold et al., 2017; Bauermeister et al., 2014; Alex Dubov et al., 2019; Goparaju et al., 2017; Hayes et al., 2019; Kubicek et al., 2015; Pérez-Figueroa et al., 2015; Rice et al., 2019; Schwartz \& Grimm, 2017), we found more specifically that the price decrease of PrEP was only related to an increase in PrEP uptake among participants with an average perceived financial situation. This might indicate that the current price reduction of PrEP (from $€ 500$ to $€ 50$ per month for the Dutch context) did not impact MSM in more unfavorable nor more favorable financial situations, likely for different reasons. MSM in a favorable perceived financial situation may use PrEP anyway, regardless of price level, because the use of PrEP does not have a substantial impact on their financial situation. On the other hand, MSM in an unfavorable perceived financial situation may find the price of $€ 50$ per month still too high and may be not be able to afford PrEP at this price. This indicates a need 
for the inclusion PrEP in health insurance or the implementation reimbursement schemes to increase PrEP uptake among less affluent MSM (Kuhns et al., 2017; Patel et al., 2017).

While having ever had PEP treatment was a significant predictor of PrEP initiation, other variables related to sexual risk behavior, such as number of sex partners in the past six months, history of STIs, condom use, and substance use were not significantly related to PrEP initiation after six months. These variables are considered key indicators for PrEP use and eligibility for PrEP, as they reflect an increased risk of HIV, and we therefore expected these to be significant predictors of PrEP initiation. A possible explanation for the lack of such a relationship is that participants in our sample overall had a high prevalence of sexual risk behaviors; most would be eligible for PrEP. Hence, their sexual risk behaviors are unlikely to distinguish between those who start taking PrEP or not (i.e, ceiling effect). This is in line with an earlier study that found that despite being an appropriate candidate for PrEP, and contemplating PrEP use, MSM do not always initiate PrEP use (Parsons et al., 2017). It was argued that PrEP initiation could be increased if PrEP providers apply motivational interviewing techniques to help MSM decide on PrEP use. It is important to recognize the role of healthcare providers in PrEP initiation. In our study we found that having had PEP treatment is related to PrEP use. This might be a result of the Dutch national guideline that instructs health care providers to encourage MSM to continue PrEP use directly after a PEP treatment (Hoornenborg \& Rijnders, 2016). This indicates that health care providers should be trained to recognize eligible candidates for PrEP and to be confident to prescribe PrEP to them.

Notably, only $45.6 \%$ of the participants in our study were using PrEP after six months follow-up. In this sample, we expected a higher PrEP uptake because of the high interest in PrEP among the participants. Moreover, the participants were recruited on a website where they could find detailed information on how to obtain PrEP, so interest and knowledge would not be limiting factors to procure PrEP for the participants in this sample. Also other studies found large gaps between interest in PrEP and PrEP uptake (Blashill et al., 2020; Parsons et al., 2017; Rolle et al., 2017). It seems that structural barriers play a larger role in explaining this gap compared to psychosocial and behavioral factors. For example, among young Latino MSM it was found that structural syndemic factors, such as poverty and unstable housing, limit PrEP uptake despite high interest in PrEP (Blashill et al., 2020). In our study we found that the perceived financial situation and the price of PrEP were the most important factors in predicting PrEP uptake. 


\section{Chapter 4}

There are a few limitations to this study. We recruited participants using convenience sampling, limiting the representativeness of this study for the whole MSM population. The sample consisted mostly of highly educated MSM who were born in the Netherlands. MSM with lower education levels and migrant MSM may face other challenges when accessing PrEP. It is important to study the specific needs of these MSM subgroups, in particular because non-Western migrant MSM in the Netherlands have an increased risk of acquiring HIV (Visser et al., 2017). Still, our study highlights that even among non-minority MSM in the Netherlands the price of PrEP and their financial situation are significant factors determining access to PrEP. The findings relate specifically to MSM with a high interest in PrEP, and are therefore mostly relevant for explaining the gap between a high interest in PrEP and a low uptake of PrEP (Hayes et al., 2019). Another limitation of this study is related to the assessment of the price of PrEP. We used the price of PrEP in pharmacies in the Netherlands to construct a variable for the price of PrEP that could be included in the regression analysis. However, some participants obtained PrEP informally (e.g., via pharmacies abroad), so the price of PrEP in pharmacies in the Netherlands may not have influenced their PrEP initiation. Monitoring of prices for PrEP at the time of the study in online pharmacies and through health care providers showed prices quite similar to the reduced price in the Netherlands, with a lower bound of $€ 30$,- in Thailand and average prices around $€ 50$,-. Another limitation is that the variable "price of PrEP" may not merely reflect the change in the price of PrEP, but may also reflect time effects. We did an additional analysis (see Appendix B of this chapter) to control for possible time effects, and found no evidence that participants were more likely to use PrEP later in time. This indicates that the effect of the variable "price of PrEP" indeed captures an effect of the price drop of PrEP.

A strength of this study is that it is the first to collect data over a time span in which the price of PrEP significantly changed, allowing us to investigate the relationship between an actual, real-world change in the price of PrEP and PrEP uptake. These findings are not only relevant for the Netherlands, but also for other countries. In 2019, PrEP was not included in reimbursement schemes in 37 (out of 53 reporting) countries in Europe, underscoring that costs of PrEP likely continue to impact PrEP use (European Centre for Disease Prevention and Control, 2019c). We further expect that the results of our study remain relevant in the future, even when the price of branded PrEP may be (further) lowered, as new types of formulation (e.g., Emtricitabine/tenofovir alafenamide; (U.S. Food and Drug Administration, 2019)) or administration (e.g., injectables, implants (Coelho et al., 2019)) may result in new pricing barriers. Pricing barriers may also continue to exist after the introduction of generic formula- 
tions of PrEP, because generic formulations of PrEP are not always substantially cheaper than branded Truvada (Poz.com, 2020).

To optimize PrEP uptake among MSM with a high interest in PrEP with limited financial resources, the cost of PrEP play an important role. The introduction of lower price generic formulations of PrEP led to an increase in PrEP uptake in the Netherlands. However, PrEP continued to be used by MSM in a favorable perceived financial situation. MSM in an unfavorable perceived financial situation may be more likely to use PrEP if it is available free of charge, through health insurance, or fully reimbursed, through a government scheme. 


\section{Appendix A}

Table 4.4. Source of PrEP procurement stratified by perceived financial situation. Please note that the total frequency exceeds the number of PrEP users, as participants could report multiple sources of procurement.

\begin{tabular}{lcccccccc}
\hline & $\begin{array}{c}\text { Via HIV- } \\
\text { positive } \\
\text { friends }\end{array}$ & $\begin{array}{c}\text { Through } \\
\text { PEP } \\
\text { treat- } \\
\text { ment }\end{array}$ & $\begin{array}{c}\text { Prescrip- } \\
\text { tion and } \\
\text { local } \\
\text { pharmacy } \\
\text { ation }\end{array}$ & $\begin{array}{c}\text { Buy- } \\
\text { ers } \\
\text { club }\end{array}$ & $\begin{array}{c}\text { Phar- } \\
\text { macy } \\
\text { abroad }\end{array}$ & $\begin{array}{c}\text { Phar- } \\
\text { macy } \\
\text { online } \\
\text { (abroad) }\end{array}$ & $\begin{array}{c}\text { PrEP } \\
\text { rearch } \\
\text { trial }\end{array}$ & Total \\
\hline $\begin{array}{l}\text { You can't make } \\
\text { ends meet } \\
\text { without bor- } \\
\text { rowing }\end{array}$ & 0 & 0 & 1 & 0 & 0 & 0 & 0 & 1 \\
$\begin{array}{l}\text { You are having } \\
\text { problems mak- } \\
\text { ing ends meet }\end{array}$ & 1 & 0 & 0 & 0 & 0 & 0 & 0 & 1 \\
$\begin{array}{l}\text { You are getting } \\
\text { by but have to } \\
\text { be careful }\end{array}$ & 0 & 1 & 10 & 0 & 5 & 0 & 1 & 17 \\
$\begin{array}{l}\text { Things are all } \\
\text { right }\end{array}$ & 0 & 4 & 33 & 1 & 8 & 1 & 2 & 49 \\
$\begin{array}{l}\text { You are doing } \\
\text { rather well }\end{array}$ & 1 & 1 & 40 & 7 & 8 & 3 & 4 & 64 \\
$\begin{array}{l}\text { You are doing } \\
\text { really well }\end{array}$ & 1 & 0 & 20 & 1 & 10 & 4 & 3 & 39 \\
\begin{tabular}{l} 
Total \\
\hline
\end{tabular} & 3 & 6 & 104 & 9 & 31 & 8 & 10 & 171 \\
\hline
\end{tabular}




\section{Appendix B}

In our analysis, the variable "price of PrEP" reflected the introduction of generic formulations of PrEP, and the related price decrease, in the Netherlands. The variable "price of PrEP" was dummy coded with " 0 " when the participant completed the T2 questionnaire at the moment when PrEP costed $€$ 500,- at Dutch pharmacies (until 01-01-2018) and with " 1 " when the participant completed the T2 questionnaire at the moment when PrEP costed $€ 50$,- at Dutch pharmacies (after 01-01-2018). However, it can be argued that this variable may not merely reflect the decrease in the price of PrEP, but instead may also reflect time effects. For example, over time MSM may become more familiar with PrEP, and may therefore initiate PrEP use.

To investigate the effect of time more precisely, we added the variable "time" in the regression model, whereby "time" reflects the month in which the participant completed the T2 survey, coded as "1" for October 2017 up to " 24 ” for September 2019. Since the variables "price of PrEP" and "time" have a high overlap regarding their underlying construct, the correlation between them is too high to include them as independent predictors at the same time in the regression model. Therefore, we conducted two logistic regression analyses including "time" but not "price of PrEP": The first logistic regression analysis covered the data $(n=84)$ during the period when the price of PrEP was high ( $€ 500,-)$. The second logistic regression analysis covered the data $(n=265)$ during the period when the price of PrEP was low ( $€ 50,-)$.

Tables 4.5 and 4.6 show the results of these two logistic regression analyses. In both analyses, "time" is not significantly related to PrEP initiation, suggesting that PrEP use does not increase over time because of, for example, becoming familiar with PrEP. These findings strengthen our interpretation that the price decrease of PrEP is related to an increase in PrEP use. 


\section{Chapter 4}

Table 4.5. Multivariable logistic regression examining correlates of PrEP initiation during the period when the price of PrEP was high (€ 500,-).

\begin{tabular}{|c|c|c|}
\hline & $\mathrm{aOR}$ & $95 \% \mathrm{CI}$ aOR \\
\hline Age & 0.98 & $0.93-1.04$ \\
\hline Number of sex partners in past 6 months & 1.02 & $0.99-1.05$ \\
\hline Perceived financial situation & 1.51 & $0.91-2.52$ \\
\hline \multicolumn{3}{|l|}{ Education level } \\
\hline Master and $\mathrm{PhD}$ & Ref & \\
\hline Bachelor & 0.31 & $0.08-1.21$ \\
\hline High school and Professional qualification & 0.75 & $0.21-2.66$ \\
\hline \multicolumn{3}{|l|}{ Relationship status } \\
\hline Single & Ref & \\
\hline In an open relationship & 0.76 & $0.23-2.49$ \\
\hline In a relationship & 1.34 & $0.15-12.28$ \\
\hline \multicolumn{3}{|l|}{ STI } \\
\hline Never had an STI & Ref & \\
\hline Had an STI in the past 12 months & 2.41 & $0.53-11.03$ \\
\hline Had an STI more than 12 months ago & 0.97 & $0.21-4.55$ \\
\hline Not used a condom the last time $\mathrm{a}^{\mathrm{a}}$ & 1.30 & $0.39-4.28$ \\
\hline Used drugs in a sexual context ${ }^{\mathrm{a}}$ & 1.10 & $0.34-3.49$ \\
\hline Ever had a PEP treatment ${ }^{\mathrm{a}}$ & 1.44 & $0.29-7.27$ \\
\hline Time $^{\mathrm{b}}$ & 0.70 & $0.36-1.35$ \\
\hline
\end{tabular}

a The reference category for these variables is "no".

b The variable "time" reflects the month in which the participant completed the T2 survey, coded as "1" for October 2017 up to "24" for September 2019.

$\chi^{2}(13, n=84)=16.14, p=.24$, Nagelkerke $R^{2}=.240$ 
Table 4.6. Multivariable logistic regression examining correlates of PrEP initiation during the period when the price of PrEP was low (€ 50,-).

\begin{tabular}{|c|c|c|}
\hline & $\mathrm{aOR}$ & $95 \% \mathrm{CI}$ aOR \\
\hline Age & 1.01 & $0.99-1.04$ \\
\hline Number of sex partners in past 6 months & 1.02 & $1.00-1.03$ \\
\hline Perceived financial situation & $1.47^{\star *}$ & $1.15-1.89$ \\
\hline \multicolumn{3}{|l|}{ Education level } \\
\hline Master \& PhD & Ref & \\
\hline Bachelor & 1.25 & $0.64-2.45$ \\
\hline High school \& Professional qualification & 1.27 & $0.66-2.44$ \\
\hline \multicolumn{3}{|l|}{ Relationship status } \\
\hline Single & Ref & \\
\hline In an open relationship & 1.36 & $0.77-2.39$ \\
\hline In a relationship & 0.32 & $0.07-1.39$ \\
\hline \multicolumn{3}{|l|}{ STI } \\
\hline Never had an STI & Ref & \\
\hline Had an STI in the past 12 months & 1.51 & $0.73-3.13$ \\
\hline Had an STI more than 12 months ago & 0.64 & $0.33-1.25$ \\
\hline Not used a condom the last time ${ }^{a}$ & 1.28 & $0.73-2.22$ \\
\hline Used drugs in a sexual context ${ }^{a}$ & 1.47 & $0.83-2.61$ \\
\hline Ever had a PEP treatment ${ }^{a}$ & $2.56^{\star}$ & $1.08-6.09$ \\
\hline Time $^{\mathrm{b}}$ & 1.04 & $0.98-1.09$ \\
\hline
\end{tabular}

a The reference category for these variables is "no".

b The variable "time" reflects the month in which the participant completed the T2 survey, coded as "1" for October 2017 up to "24" for September 2019.

${ }^{*} p<.05$

${ }^{* *} p<.01$

$\chi^{2}(13, n=265)=35.83, p<.001$, Nagelkerke $R^{2}=.169$ 


\section{CHAPTER 5 Quality of sex life and perceived sexual pleasure of informal PrEP users in the Netherlands}

This chapter is based on:

Van Dijk, M., De Wit, J.B.F., Guadamuz, T., Martinez, J.E., \& Jonas, K.J. (2020). Quality of sex life and perceived sexual pleasure of informal PrEP users in the Netherlands [Manuscript submitted for publication]. 


\begin{abstract}
Next to its benefits for HIV prevention, PrEP may have psychosocial benefits relating to improved quality of sex life. The aim of the current study was to investigate the onset of changes in quality of sex life and sexual pleasure of PrEP users in the first months of commencing PrEP use. Moreover, we investigated what factors were related to the quality of sex life of PrEP users. We recruited 145 participants via the Dutch PrEP-advocacy website PrEPnu.nl, and they received follow-up questionnaires after three and six months. We found that PrEP users reported an increase in the quality of their sex life, which was related to reduced fear of HIV since they started using PrEP, but not to decreased condom use. PrEP users were more interested in experimenting with sex practices, but they did not always feel more desirable as a sex partner because of PrEP use. Health care providers and health promotion campaigns could emphasize the positive effects of PrEP on the quality of sex life, in addition to the HIV-preventive effects of PrEP, to decrease PrEP stigma and increase PrEP uptake.
\end{abstract}




\section{Introduction}

Men who have sex with men (MSM) are disproportionally affected by HIV. In the Netherlands, about two-third of HIV infections are among MSM, and in the European Union sex between men is the predominant mode of HIV transmission (European Centre for Disease Prevention and Control, 2019b). Pre-exposure prophylaxis (PrEP) is an effective biomedical HIV-prevention tool (Fonner et al., 2016; Grant et al., 2010, 2014; J. M. Molina et al., 2017), that has shown promising results in decreasing new HIV infections among MSM (Grulich et al., 2018; Public Health England, 2019; Smith et al., 2020). Initially, PrEP uptake was slow (Kirby \& Thornber-Dunwell, 2014; Parsons et al., 2017; Rana et al., 2018), but uptake is increasing in contexts where PrEP became more easily available and affordable, for example in San Francisco and Australia (Medland et al., 2020; Volk et al., 2020), but also in European countries like Germany and Belgium (Mysior et al., 2020; Vuylsteke et al., 2019). PrEP uptake and continuous use may further increase if psychosocial benefits of PrEP are better understood and promoted.

Many MSM experience fear of HIV infection (Koester et al., 2017; Prestage et al., 2012; Whitfield et al., 2019), which affects their quality of sex life and perceived sexual pleasure, and creates barriers for HIV testing (Dowson et al., 2012). It has been found that PrEP reduces HIV-related fear (Collins et al., 2017; Hojilla et al., 2016; Keen et al., 2020; Koester et al., 2017; Moeller et al., 2020; Whitfield et al., 2019), and consequently it may increase the quality of MSM's sex life. While it has been suggested that PrEP may have benefits for quality of sex life in addition to its benefits for HIV prevention (Calabrese \& Underhill, 2015; Grant \& Koester, 2016; Race, 2016), there are only a limited amount of studies that investigated the relationship between PrEP use and quality of sex life, and to our best knowledge there are no studies that investigated the onset of such effects after starting to use PrEP. In an analysis of Facebook posts of PrEP users in the USA, it was found that PrEP enables PrEP users to explore sexual desires and practices in their search for pleasurable sexual relations (Da Silva-Brandao \& Ianni, 2020). In a qualitative study in France, PrEP users in a community-based trial reported an increase in sexual pleasure and quality of sexual life (Mabire et al., 2019). PrEP users related improvements in sexual pleasure to greater feelings of sexual freedom, and the flexibility to (not) use condoms (Mabire et al., 2019). Condomless anal intercourse has already been related to greater sexual pleasure (Carballo-Diéguez et al., 2011), even before the introduction of PrEP. Two systematic reviews found that condom use significantly decreases among PrEP users (Freeborn \& Portillo, 2018; Traeger et al., 2018). In sum, there is initial evidence that PrEP 


\section{Chapter 5}

use also has (positive) psychosocial effects due to change of condom use, increased sexual exploration and perceived sexual freedom. So far, PrEP use is mostly studied in the context of demonstration trials, in which PrEP users received counseling and medical supervision. In the current study, we assessed the experiences of PrEP users in the Netherlands outside of clinical trials. At the time of our study, many PrEP users obtained PrEP via informal channels (i.e. pharmacies abroad), because formal PrEP services were not implemented yet.

The aim of the current study was to document the onset of changes in behaviors and attitudes of PrEP users in a longitudinal design (first six months of PrEP use) and to investigate factors that are related to the quality of sex life of PrEP users. As prior qualitative studies point out, we expect that less fear of HIV and a decrease in condom use are related to an improved quality of sex life among PrEP users. We also expect that greater feelings of sexual freedom, as reflected by experimenting with sex practices or recreational substance use, are related to an improved quality of sex life. We first analyzed whether these behaviors and attitudes change in the first months of commencing PrEP users. Next, we analyzed how the behaviors and attitudes are related to the quality of sex life.

\section{Methods}

\section{Participants and procedure}

Between February 2017 and March 2019, participants were recruited via the Dutch PrEP-advocacy website PrEPnu.nl. This website provides information about using and obtaining PrEP aimed at potential PrEP users in the Netherlands. Participants received a follow-up questionnaire via email after three (T1) and six months (T2). All participants who completed at least two questionnaires (Baseline T0 + T1/T2) were entered into a raffle to win a $€ 100$,- gift card. This study was approved by the Ethics Review Committee Psychology and Neuroscience of Maastricht University (ERCPN-174_10_12_2016). The full details of the methods of this study have been described elsewhere (Van Dijk et al., 2020).

Participants younger than 18 years old or living with HIV were excluded from participation. For the current analysis, we only included participants who reported using PrEP at T1 and T2, regardless of their PrEP use at baseline (T0). We did not include data from T0, because not all relevant items for the current analysis were assessed at T0. 


\section{Materials}

The questionnaires were administered online using Qualtrics. The questionnaires were offered in Dutch and English, and included the following assessments.

\section{Sociodemographic characteristics}

In the baseline questionnaire (T0) participants were asked to indicate their gender (gender assigned at birth and current gender), age, relationship status, educational level, financial situation, country of birth, and country of residence. We assessed perceived financial situation with a 6-point scale; (1) 'you can't make ends meet without borrowing', (2) 'you are having problems making ends meet', (3) 'you are getting by but have to be careful', (4) 'things are all right', (5) 'you are doing rather well', and (6) 'you are doing really well'.

\section{Quality of sex life and quality of life}

At T1 and T2, we asked participants whether their perceived quality of sex life changed since they started taking PrEP, with responses indicated on a 5-point scale ranging from (1) 'a lot worse' to (5) 'a lot better'. We included a separate item to assess perceived change in quality of life in general to investigate whether quality of sex life explains additional variance.

\section{PrEP-regimen and consequences of PrEP use}

We asked current PrEP users whether they used PrEP daily or on demand. At T1 and T2, we also asked them to indicate their agreement, using a 5-point scale ranging from (1) strongly disagree to (5) strongly agree, with the following five items that were phrased for the period since they started using PrEP: 'I feel less anxious when having sex', 'I feel more safe when having sex', 'I feel I am a more desirable sex partner because of PrEP', 'I feel more interested in experimenting with novel sex practices', and 'I am interested in using (novel) recreational drugs while having sex'. Due to the conceptual overlap of the first two items, (less) anxious and (more) safe, $(r=.72$ at T1 and $r=.57$ at T2), we computed a new variable based on the mean of these two items.

\section{Condom use}

To assess condom use, we asked participants whether their condom use changed since they started taking PrEP on a scale from (1) 'a lot lower than before' to (5) 'a lot higher than before'.

\section{Data analysis}

We analyzed the data using IBM SPSS Statistics version 26. To assess changes in quality of sex life, condom use, and the PrEP-related items over a period of three months, we conducted a 


\section{Chapter 5}

repeated measures ANOVA to compare the two measurements (T1 and T2) of each variable. To assess if quality of life, condom use and the PrEP-related items were related to quality of sex life, we conducted a linear regression analysis with quality of sex life at T2 as dependent variable. In addition, we conducted a mediation analysis to assess if reduced fear of HIV was associated with improved quality of sex life as mediated by decreased condom use, using 5000 bootstrapped standard errors in PROCESS version 3 for SPSS (Hayes, 2017).

\section{Results}

\section{Participant characteristics and PrEP regimen}

Table 5.1 shows the participant characteristics. All 145 participants were cisgender males. Their mean age was 43 years (range: 20 - 71). Most participants were single (70; 48.3\%) or in an open relationship $(66 ; 45.5 \%)$. Overall, the sample was relatively high educated ( 95 participants (65.6\%) had a Bachelor degree or higher) and in a comfortable perceived financial situation (128 participants (88.4\%) evaluated their financial situation as "all right" or higher).

More than half of the participants used PrEP daily (T1: 109 participants (75.2\%), T2: 101 participants (69.7\%)), and the remainder used PrEP on demand (T1: 36 (24.8\%), T2: $44(30.3 \%)$ ). Recreational drug use in a sexual context was common: 66 participants (45.5\%) indicated to have used XTC (ecstasy/MDMA) while on PrEP, and 61 participants (42.1\%) indicated to have used GHB/GHL. Use of other drugs are reported in table 5.1.

\section{Changes in behavior and attitudes}

Table 5.2 shows the descriptive statistics of quality of sex life, condom use, and the PrEP-related items, including the results of the repeated measures ANOVA. PrEP users reported improvements in their perceived quality of sex life with an average score of $4.24(S D=0.71)$ at $\mathrm{T} 1$. The improvements in quality of sex life maintained over time; the score at T2 did not differ from the score at T1 $(M=4.19, S D=0.69 ; F<1)$. PrEP had less impact on quality of life in general. Compared to quality of sex life, the scores on quality of life in general were closer to the middle value (3), indicating that perceived quality of life was "the same" as before using PrEP. At T2, perceived improvements in quality of life were lower than at $\mathrm{T} 1(F(1,144)=$ $\left.8.78, p=.004, \eta_{\mathrm{p}}{ }^{2}=.06\right)$, indicating that improvements in quality of life in general did not maintain over time. 
Table 5.1. Frequencies of participant characteristics $(N=145)$.

Age (years; mean, range)

$43(20-71)$

Born in the Netherlands

$119(82.1 \%)$

Living in the Netherlands

$140(96.6 \%)$

Perceived financial situation

You can't make ends meet without borrowing

$1(0.7 \%)$

You are having problems making ends meet

$2(1.4 \%)$

You are getting by but have to be careful

$14(9.7 \%)$

Things are all right

$43(29.7 \%)$

You are doing rather well

$52(35.9 \%)$

You are doing really well

$33(22.8 \%)$

Education level

No tertiary education

$21(14.5 \%)$

Professional / vocational qualification

$29(20.0 \%)$

Bachelor degree

$43(29.7 \%)$

Master degree

$43(29.7 \%)$

$\mathrm{PhD}$ degree

$9(6.2 \%)$

Relationship status

Single

$70(48.3 \%)$

In a relationship

$9(6.2 \%)$

In an open relationship

$66(45.5 \%)$

Used this drug in a sexual context since PrEP initiation:

3-MMC

$14(9.7 \%)$

4-MEC

$2(1.4 \%)$

4-FA

$3(2.1 \%)$

Cocaine

$19(13.1 \%)$

Crystal meth

$9(6.2 \%)$

GHB/GHL

$61(42.1 \%)$

Ketamine

$34(23.4 \%)$

Methoxetamine (MXE)

$6(4.1 \%)$

XTC (ecstasy / MDMA)

$66(45.5 \%)$ 


\section{Chapter 5}

Table 5.2. Descriptive statistics and repeated measures ANOVA to compare the scores on the variables of interest between the two time points.

\begin{tabular}{|c|c|c|c|c|c|c|c|}
\hline & $M \mathrm{~T} 1$ & $S D \mathrm{~T} 1$ & $M \mathrm{~T} 2$ & $S D$ T2 & $F$ & $p$ & $\eta_{p}^{2}$ \\
\hline Quality of life ${ }^{1}$ & 3.76 & 0.72 & 3.59 & 0.66 & 8.78 & .004 & .06 \\
\hline Quality of sex life ${ }^{1}$ & 4.24 & 0.71 & 4.19 & 0.69 & 0.94 & .33 & .01 \\
\hline $\begin{array}{l}\text { I feel less anxious } \\
\text { / more safe when } \\
\text { having sex }{ }^{2}\end{array}$ & 4.53 & 0.86 & 4.65 & 0.72 & 1.84 & .18 & .01 \\
\hline $\begin{array}{l}\text { I feel I am a more } \\
\text { desirable sex partner } \\
\text { because of } \text { PrEP}^{2}\end{array}$ & 3.08 & 1.21 & 3.04 & 1.24 & 0.09 & .77 & .001 \\
\hline $\begin{array}{l}\text { I feel more interested } \\
\text { in experimenting } \\
\text { with novel sex prac- } \\
\text { tices }^{2}\end{array}$ & 3.47 & 1.20 & 3.70 & 1.13 & 4.88 & .03 & .03 \\
\hline $\begin{array}{l}\text { I am interested in } \\
\text { using (novel) recre- } \\
\text { ational drugs while } \\
\text { having sex }{ }^{2}\end{array}$ & 2.01 & 1.29 & 2.04 & 1.28 & 0.17 & .68 & .001 \\
\hline Condom use ${ }^{3}$ & 2.19 & 0.79 & 2.03 & 0.78 & 9.49 & .002 & .06 \\
\hline
\end{tabular}

Degrees of freedom $=1,144$

${ }^{1}$ Change in quality of life and quality of sex life since taking PrEP on a scale from (1) 'a lot worse' to (5) 'a lot better'.

${ }^{2}$ All items began with 'Since I started using PrEP.... Participants indicated their agreement using a 5 -point scale ranging from (1) strongly disagree to (5) strongly agree.

${ }^{3}$ Change in condom use since taking PrEP on a scale from (1) 'a lot lower than before' to (5) 'a lot higher than before'.

PrEP users reported high agreement with feeling less anxious when having sex since they started PrEP use, both at T1 and T2. PrEP use however did not make them feel more desirable as a sex partner at T1 nor T2. PrEP use did make them somewhat more interested in experimenting with sex practices, and this increased over time $\left(F(1,144)=4.88, p=.03, \eta_{\mathrm{p}}{ }^{2}\right.$ $=.03)$. Notably, this increased interest in sexual experimentation did not involve the use of recreational drugs, as PrEP users indicated that they were not interested in using new types of drugs while having sex. Furthermore, PrEP users reported a decrease in perceived condom use at T1 since they started using PrEP, and condom use further decreased at T2 $(F(144,1)$ $\left.=9.49, p=.002, \eta_{\mathrm{p}}^{2}=.06\right)$. 
Table 5.3. Multivariable regression analysis with quality of sex life at $\mathrm{T} 2$ as outcome variable.

\begin{tabular}{|c|c|c|c|c|c|}
\hline & $B$ & S.E. & Beta & $t$ & $p$ \\
\hline (Constant) & 0.44 & 0.53 & & 0.83 & 0.41 \\
\hline Quality of sex life (T1) & 0.31 & 0.08 & 0.32 & 3.65 & $<0.001$ \\
\hline Condom use (T1) & -0.01 & 0.08 & -0.01 & -0.15 & 0.88 \\
\hline Condom use (T2) & 0.00 & 0.08 & 0.00 & 0.03 & 0.98 \\
\hline $\begin{array}{l}\text { I feel less anxious / more } \\
\text { safe when having sex (T1) }\end{array}$ & 0.01 & 0.06 & 0.01 & 0.13 & 0.90 \\
\hline $\begin{array}{l}\text { I feel less anxious / more } \\
\text { safe when having sex (T2) }\end{array}$ & 0.17 & 0.07 & 0.18 & 2.52 & 0.01 \\
\hline $\begin{array}{l}\text { I feel more interested in } \\
\text { experimenting with novel } \\
\text { sex practices (T1) }\end{array}$ & 0.02 & 0.05 & 0.04 & 0.47 & 0.64 \\
\hline $\begin{array}{l}\text { I feel more interested in } \\
\text { experimenting with novel } \\
\text { sex practices (T2) }\end{array}$ & 0.02 & 0.05 & 0.03 & 0.38 & 0.71 \\
\hline $\begin{array}{l}\text { I feel I am a more desira- } \\
\text { ble sex partner because of } \\
\text { PrEP (T1) }\end{array}$ & -0.02 & 0.05 & -0.03 & -0.42 & 0.67 \\
\hline $\begin{array}{l}\text { I feel I am a more desira- } \\
\text { ble sex partner because of } \\
\text { PrEP (T2) }\end{array}$ & 0.08 & 0.04 & 0.15 & 1.88 & 0.06 \\
\hline Quality of life (T1) & 0.01 & 0.08 & 0.01 & 0.11 & 0.91 \\
\hline Quality of life (T2) & 0.37 & 0.08 & 0.35 & 4.53 & $<0.001$ \\
\hline $\begin{array}{l}\text { I am interested in using } \\
\text { (novel) recreational drugs } \\
\text { while having sex (T1) }\end{array}$ & -0.08 & 0.05 & -0.15 & -1.51 & 0.13 \\
\hline $\begin{array}{l}\text { I am interested in using } \\
\text { (novel) recreational drugs } \\
\text { while having sex (T2) }\end{array}$ & 0.07 & 0.05 & 0.13 & 1.27 & 0.21 \\
\hline
\end{tabular}

$R^{2}=.47, F(13,144)=8.92, p<.001$

\section{Predictors of quality of sex life}

To investigate what factors were related to quality of sex life at T2, we conducted a linear regression analysis (Table 5.3). Significant predictors of quality of sex life at T2 were quality of sex life at T1 $(B=0.31, t=3.65, p<.001)$, quality of life in general at T2 $(B=0.37, t=4.53, p$ $<.001)$, and feeling less fear of HIV at T2 $(B=0.17, t=2.52, p=.01)$. 


\section{Chapter 5}

In order to better understand the relationship between reduced fear of HIV and increased quality of sex life, we assessed if this relationship was mediated by condom use (Figure 5.1). These analyses showed no indirect effect of condom use $(b=0.02,95 \% \mathrm{CI}=-0.01-0.06)$, but did show a direct effect of fear of HIV on quality of sex life $(b=0.23, t(142)=2.98, p=.003)$ that was not mediated by condom use.

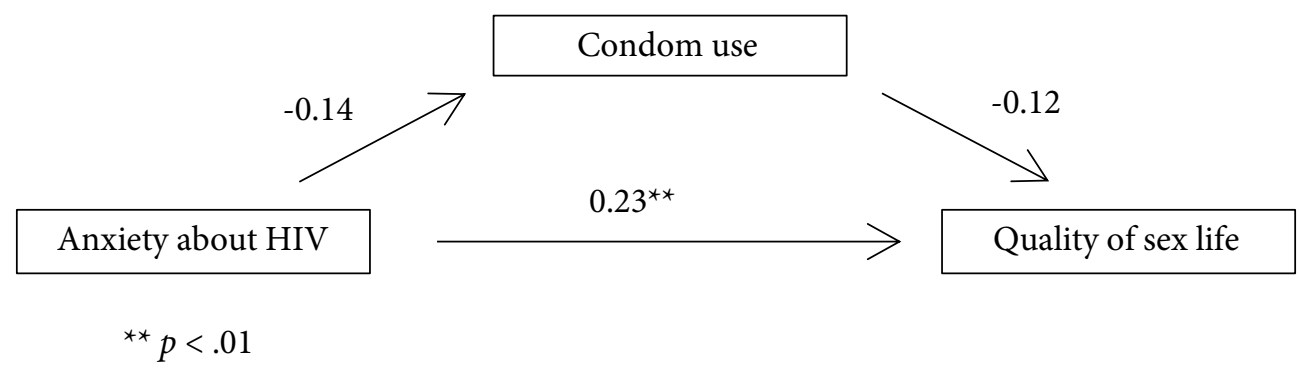

Figure 5.1. Mediation analysis investigating the relationship between fear of HIV and quality of sex life mediated by condom use, all at $\mathrm{T} 2$.

\section{Discussion}

The aim of the current study was to assess the onset of changes in perceived quality of sex life and sexual pleasure of PrEP users in their first six months of PrEP use. We also assessed factors that were related to the quality of sex life of PrEP users. PrEP users reported an increase in quality of sex life, and, to a lesser extent, an increase in quality of life in general. Similar to earlier studies (Collins et al., 2017; Hojilla et al., 2016; Keen et al., 2020; Koester et al., 2017; Moeller et al., 2020; Whitfield et al., 2019), PrEP users also reported less fear of HIV when having sex since they started using PrEP. This reduced fear was positively related to improvements in quality of sex life. The relationship between fear of HIV and quality of sex life was not found to be mediated by changes in condom use.

Regarding sexual pleasure, we found that PrEP users become somewhat more interested in experimenting with sex practices since they started using PrEP, and this interest increased over time. Although we did not ask our participants for any specification of these sex practices, earlier qualitative studies found that PrEP users indicated that they were more likely to switch their sexual position from top to bottom (Mabire et al., 2019; Quinn et al., 2019), and PrEP users were more likely to have attended sex parties (Meunier \& Siegel, 2019). Also a 
quantitative study among 1,500 MSM in Australia found that PrEP users had a more 'adventurous' sex life as they were more likely to have engaged in group sex and had higher number of sex partners (Prestage et al., 2019). Overall, we found that drug use in the context of sex was high, similar to other studies that reported high levels of recreational drug use or chemsex (the use of drugs while having sex) among PrEP users (Closson et al., 2018; Gafos et al., 2019; Nöstlinger et al., 2020; Roux et al., 2018). However, PrEP users in our study did not have an increased interest in using (novel) recreational drugs while having sex since they started using PrEP. Notably, a study among PrEP users in a demonstration trial in the Netherlands found that (problematic) drug use actually decreased after PrEP initiation (Achterbergh et al., 2020). The authors propose the possible explanation that PrEP users in the demonstration trial received counseling and motivational interviewing. In our study, no decrease in drug use was found. A possible explanation is that since we recruited participants in a context where formal PrEP services were not fully implemented yet, PrEP users may not have received counseling, and therefore did not change their drug use behavior.

PrEP users reported a decrease in condom use, and this seemed to continue over time. PrEP users overall did not report a change in feeling a more desirable sex partner since they started using PrEP, while in the literature changes have been found in both directions: Some PrEP users feel being a less desirable sex partner, as they have encountered stigmatizing reactions from potential sex partners (Brooks et al., 2019; Grace et al., 2018). Other PrEP users feel being a more desirable sex partner, as they are seen as having a lower risk of having HIV (Martinez \& Jonas, 2019).

The findings of our study contribute to the discourse of psychosocial benefits and sex-positive effects of PrEP. Initially, PrEP users were stigmatized, as reflected by the use of such terms as "Truvada whores" (Calabrese \& Underhill, 2015). However, a recent study in Australia, where PrEP is widely available, found that positive views of PrEP were twice as common than negative views of PrEP (Philpot et al., 2020). Researchers have advocated to focus on the empowerment regarding sexual health and how PrEP fits in the complete panel of HIV and STI prevention strategies (Rojas Castro, Delabre, \& Molina, 2019). To increase PrEP uptake and continuous use, health promotion campaigns should include the positive effects of PrEP, such as the improved quality of sex life and reduced anxiety for HIV. For example, a PrEP campaign in Chicago that underlined sexual pleasure was found to increase PrEP awareness and engagement (Dehlin et al., 2019; Keene et al., 2020). 


\section{Chapter 5}

This study has several limitations. We recruited participants by convenience sampling through the PrEP advocacy website PrEPnu.nl, which limits the representativeness of the sample. Especially at the start of our recruitment, in early 2017, awareness and use of PrEP was still limited in the Netherlands. Hence, it was practical to recruit participants, that is (potential) PrEP users, through channels for this population group. By using this platform, we may have in particular recruited motivated and well-informed (potential) PrEP users, and we may have missed (potential) PrEP users who find PrEP information via other sources, such as social medial or their healthcare providers. As PrEP use is becoming more common in many countries, future studies may want to recruit a wider range of PrEP users. Another limitation is that the data is self-reported. While participants could complete the questionnaire anonymously, the responses could still be affected by social desirability biases. A final limitation is that we did not ask the questions to MSM who were not using PrEP, so we cannot compare quality of sex life of PrEP users with non-users. We asked PrEP users to indicate changes in quality of sex life since they started taking PrEP, so we can only make inferences about the effect of PrEP based on these self-reported changes.

To conclude, we found that early PrEP users in the Netherlands reported an increase in the quality of their sex life, which was associated with reduced fear of HIV while having sex since they started using PrEP. PrEP users also were more interested in experimenting with novel sex practices, but they did not always feel more desirable as a sex partner because of PrEP use. Health care providers and health promotion campaigns could point out the positive effects of PrEP on the quality of sex life, in addition to the HIV-preventive effects of PrEP, to decrease PrEP stigma and increase PrEP uptake and continuous use. 
QUALity OF SEX LIFE AND PERCEIVED SEXUAL PLEASURE 


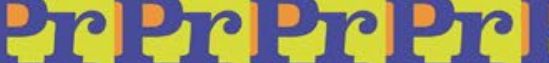

IC DIC DYC

DO

DCo

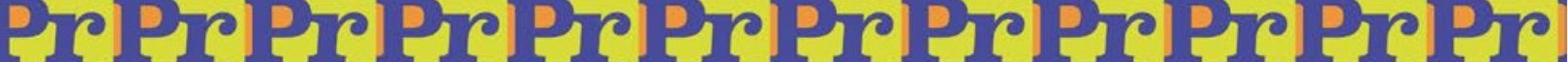

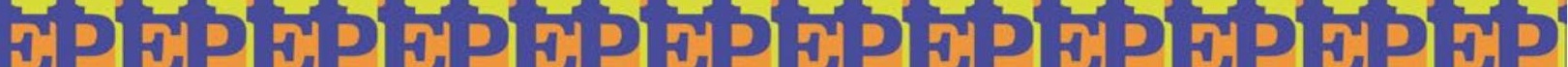
10 (1) 


\section{CHAPTER 6 Bridging the serodivide: Attitudes of PrEP users towards sex partners living with HIV}

This chapter is based on:

Van Dijk, M., De Wit, J.B.F., Guadamuz, T., Martinez, J.E., \& Jonas, K.J. (2020). Bridging the serodivide: Attitudes of PrEP users towards sex partners living with HIV [Manuscript submitted for publication]. 


\begin{abstract}
The introduction of biomedical HIV prevention methods, such as pre-exposure prophylaxis (PrEP), holds the potential to overcome the serodivide. We investigated the attitudes of PrEP users towards having sex with partners living with HIV. PrEP users in the Netherlands were recruited online and completed three questionnaires over a period of six months. We investigated changes over time in feelings of fear of HIV, comfort, and attitudes towards condom use when having sex with men living with HIV. A majority of PrEP users in our sample (up to $71.6 \%$ ) had sex with men living with HIV. Feeling comfortable to have sex with men living with HIV did not change over time, but was already at a high level at T1. Most importantly, feeling safe not to use condoms with HIV positive partners significantly increased, and did so in a rather short period of time after the onset of PrEP use (3-6 months). Taken together, the findings suggest that that PrEP may contribute to decreasing the serodivide between MSM rather quickly after the onset of PrEP use.
\end{abstract}




\section{Introduction}

The division between HIV-positive and HIV-negative men who have sex with men (MSM) has been described as the 'serodivide'; defined as the avoidance of sex with a partner of a different (serodiscordant) HIV status (Koester et al., 2018). The serodivide is driven by concerns about HIV transmission, resulting in serosorting, an HIV-risk reduction strategy of limiting one's sex partners the others of the same HIV status (Davis et al., 2006; Eaton et al., 2009; Golden et al., 2008). Furthermore, the serodivide is driven by HIV-related stigma and lack of contact with people living with HIV, indicative of a broad social exclusion (Murphy et al., 2015; Smit et al., 2012). HIV-stigma has a negative impact on the mental health of gay men living with HIV as well as HIV-negative gay men (Courtenay-Quirk et al., 2006; Starks et al., 2013; Stutterheim et al., 2009).

The introduction of biomedical HIV prevention, such as pre-exposure prophylaxis (PrEP) and treatment-as-prevention (TasP), holds the potential to overcome the serodivide between serodiscordant MSM and to reduce HIV stigma (Brisson \& Nguyen, 2017; Grant \& Koester, 2016; Haas et al., 2017; Jaspal \& Daramilas, 2016; Malone et al., 2018; Persson, 2016). While there is initial evidence of this potential, it is important to know when such a serodivide reduction becomes psychologically and behaviorally relevant after the onset of PrEP use.

In a qualitative study among young PrEP users in an urban setting, PrEP users were more likely to have dates with men living with HIV after starting PrEP because of a decreased fear for HIV (Koester et al., 2018). An experimental study found that PrEP users rated the dating profiles of men living with HIV as equally attractive as dating profiles of HIV-negative men, while MSM with no history of PrEP use rated dating profiles of men living with HIV as less attractive (Golub et al., 2018). Moreover, while MSM who did not use PrEP had a higher intention to use condoms with sex partners living with HIVs, PrEP users had the same (and overall lower) level of intention to use condoms with HIV-positive and HIV-negative partners (Golub et al., 2018). While these initial findings are promising, more empirical research is needed to investigate the potential effect of PrEP on decreasing the serodivide in other contexts and if this effect is persistent over time.

There are several factors that may play a role in the potential of PrEP to facilitate sexual interactions between serodiscordant sex partners. Firstly, PrEP users have been found to report increased comfort and confidence in connecting with other men sexually, particularly with men living with HIV (Storholm et al., 2017). Secondly, gay men in serodiscordant relationships 


\section{Chapter 6}

have been found to expect that PrEP use would increase their sexual comfort, as they would feel less fear of HIV (Brooks et al., 2011). Thirdly, PrEP users have reported reduced fear of HIV (Collins et al., 2017; Hojilla et al., 2016; Keen et al., 2020; Koester et al., 2017; Whitfield et al., 2019). It is expected that this reduced fear of HIV may be related to decreased condom use, as PrEP users may feel sufficiently protected against HIV by PrEP. Fourthly, compared to gay men who were not using PrEP, PrEP users felt more comfortable having condomless anal sex with men living with HIV, especially when the partner living with HIV had an undetectable viral load (Holt, Draper, et al., 2018). Hence, (non-)condom use can serve as an indicator of PrEP user's fear of HIV and sexual comfort when having sex with a partner living with HIV.

The aim of the current study was to investigate the onset and the change in attitudes of PrEP users regarding having sex with men living with HIV. We in particular investigated whether PrEP users who have sex with men living with HIV over time feel less fear of HIV, feel more comfortable when having sex with men living with HIV, and feel safe to not use condoms. To investigate these questions, we recruited PrEP users in the Netherlands at a time (2017 - 2019) when PrEP availability was still limited in the Netherlands, and not formally implemented yet. As a consequence, most PrEP users were informal PrEP users, meaning that they either procured PrEP via informal channels (i.e. pharmacies abroad or pill sharing of people living with HIV), or had limited access to PrEP-related medical services (Buttram, 2018). We followed early PrEP users over a period of six months, and this longitudinal assessment allowed us to investigate changes over time in fear of HIV, feeling comfort in having sex with men living with HIV, and feeling safe to not use condoms.

\section{Methods}

\section{Participants and procedure}

We recruited participants via the Dutch PrEP-advocacy website PrEPnu.nl, between February 2017 and March 2019. After the intake questionnaire (T0), every participant received a follow-up questionnaire via email after three (T1) and six months (T2). Participants who did not complete the T1 questionnaire were still encouraged to complete the T2 questionnaire. All participants who completed at least two questionnaires $(\mathrm{T} 0+\mathrm{T} 1 / \mathrm{T} 2)$ were entered into a raffle to win a $€ 100$,- gift card. The Ethics Review Committee Psychology and Neuroscience of Maastricht University approved this study (ERCPN-174_10_12_2016). The full details of the methods of this study have been described before (Van Dijk et al., 2020). 
Participants younger than 18 years or living with HIV were excluded from participation. For the current analysis, we only included participants who reported using PrEP both at T1 (3 months after baseline) and T2 (6 months after baseline), regardless of their PrEP use at T0 to be able to assess the onset of serodivide decrease effects. We did not include data from T0, because not all relevant items for this analysis were assessed at $\mathrm{T} 0$.

\section{Materials}

The questionnaires were administered online using Qualtrics; participants could not click back to previous questions. The questionnaires were offered in Dutch and English. We asked current PrEP users whether they used PrEP daily, on demand, or recreationally (i.e. season based (Elsesser et al., 2016; Hojilla et al., 2016; Underhill et al., 2018)). At T1 and T2, PrEP users were asked if they have had sex with HIV-positive men (yes/no). If yes, we asked them to indicate their agreement, using a 5-point scale ranging from (1) strongly disagree to (5) strongly agree, with on the following three items which were asked with respect to the period since they started using PrEP: "I feel more comfortable when I have sex with HIV positive men", "I have less fear of HIV when I have sex with HIV positive men", and "I feel safe to not use condoms with HIV positive sex partners".

\section{Data analysis}

We analyzed the data using IBM SPSS Statistics version 26. A chi-square test was conducted to assess any differences between T1 and T2 in the percentage of PrEP users who had sex with HIV-positive men and in PrEP regimen. To assess any change in attitudes between T1 and T2, we conducted a repeated measures analysis of variance (ANOVA) for each variable.

\section{Results}

\section{Participant characteristics and prevalence of sexual encounters with men living with HIV}

There were 183 PrEP users in our sample who answered the question whether they had sex with men living with HIV at both points of assessment. At T1, 121 (66.1\%) PrEP users indicated to have sex with men living with HIV. At T2, 131 (71.6\%) PrEP users reported having sex with men living with HIV, which is a significant higher amount compared to T1 $\left(\chi^{2}(1\right.$, $183)=89.92, p<.001)$. For the analyses below, we only use data of the PrEP users who had sex with men living with HIV at both assessments $(N=112)$ to correct for incidental sexual experiences with men living with HIV at either T1 or T2. 


\section{Chapter 6}

All of these 112 participants were cisgender males, with a mean age of 45 years (range: 22 - 71). The majority of the participants used PrEP daily (T1: 67 (59.8\%) participants, T2: 61 (54.5\%) participants), followed by PrEP on demand (T1: 28 (25.0\%) participants, T2: 34 (30.4\%) participants), and recreationally / season based (17 (15.2\%) participants at both T1 and $\mathrm{T} 2$ ).

\section{Changes in attitudes}

Table 6.1 shows the descriptive statistics of the attitude items, including the test results of the repeated measures ANOVA. The scores on the variables 'feeling more comfortable having sex with HIV positive men' and 'having less fear of HIV when I have sex with HIV positive men' did not change between $\mathrm{T} 1$ and $\mathrm{T} 2(F<1)$, and were already relatively high at $\mathrm{T} 1$. Scores on the variable 'feeling safe to not use condoms with HIV positive sex partners' increased significantly from $\mathrm{T} 1$ to $\mathrm{T} 2\left(F(1,111)=5.57, p=.02, \eta_{\mathrm{p}}{ }^{2}=.048\right)$.

Table 6.1. Descriptive statistics and repeated measures ANOVA to compare the scores on the variables of interest between the two assessments (T1 and T2).

\begin{tabular}{lcccccccc}
\hline & $M \mathrm{~T} 1$ & $S D \mathrm{~T} 1$ & $M \mathrm{~T} 2$ & $S D \mathrm{~T} 2$ & $F$ & $\mathrm{df}$ & $p$ & $\eta_{\mathrm{p}}{ }^{2}$ \\
\hline $\begin{array}{l}\text { I have less fear of HIV } \\
\text { when I have sex with }\end{array}$ & 4.33 & 1.06 & 4.33 & 1.01 & $<0.001$ & 1,111 & 1.00 & $<.001$ \\
$\begin{array}{l}\text { HIV positive men } \\
\begin{array}{l}\text { I feel more comfort- } \\
\text { able when I have sex }\end{array}\end{array}$ & 4.16 & 1.11 & 4.26 & 1.08 & 0.58 & 1,111 & .45 & .005 \\
$\begin{array}{l}\text { with HIV positive } \\
\text { men }\end{array}$ & & & & & & & & \\
$\begin{array}{l}\text { I feel safe to not } \\
\text { use condoms with }\end{array}$ & 3.70 & 1.39 & 4.01 & 1.23 & 5.57 & 1,111 & .02 & .048 \\
$\begin{array}{l}\text { HIV-positive sex } \\
\text { partners }\end{array}$ & & & & & & & & \\
\hline
\end{tabular}

All items began with 'Since I started using PrEP.... Participants indicated their agreement using a 5 -point scale ranging from (1) strongly disagree to (5) strongly agree.

\section{Discussion}

The aim of this study was to investigate changes in attitudes of PrEP users regarding sex with men living with HIV. We in particular investigated whether PrEP users who had sex with HIV-positive men over time had less fear of HIV, felt more comfortable having sex with men with HIV, and felt safe to not use condoms. We found that about 70\% of PrEP users $(66.1 \%$ at $\mathrm{T} 1$ and $71.6 \%$ at T2) indicated they had sex with men living with HIV. Feeling comfortable 
having sex with men living with HIV did not change over time, but was already at a high level at T1. Most importantly, feeling safe not to use condoms with HIV positive partners significantly increased, and did so in a rather short period of time after the onset of PrEP use (3-6 months)

Putting our findings into context, the amount of PrEP users having sex with men living with HIV was a relatively large proportion of the overall sample, and much higher than what was found in another study of MSM in Canada (Wang et al., 2020). This study found that only $17 \%$ of PrEP users indicated having sex with men living with. There was also less serosorting among PrEP users, as PrEP users were more likely to have sex with men living with HIV than those who did not use PrEP. An increase in having sex with men living with HIV over time was also found in another longitudinal study with a longer timeframe (up to 18 months) (Zablotska et al., 2019) while our results show an earlier onset of a serodivide decrease. The findings of our and earlier studies point to the potential that PrEP use may help overcome the divide between serodiscordant MSM.

PrEP users reported a robust decrease in fear of HIV and feeling more comfortable having sex with partners living with HIV, and this did not change over a period of three months. While reduced fear of HIV and increased sexual comfort have been reported in qualitative studies (Collins et al., 2017; Hojilla et al., 2016; Koester et al., 2017; Storholm et al., 2017), our study, to the best of our knowledge, is the first quantitative study to report this reduced fear and increased comfort in a longitudinal design with informal PrEP users. Our findings are similar to those of an earlier longitudinal study with formal PrEP users in the United States (Whitfield et al., 2019), however the focus of our study is specifically on sexual interaction with sex partners living with HIV. In addition to the reduced fear of HIV, we found that PrEP users felt safe to not use condoms with sex partners living with HIV, and this increased over time. This suggests that, over time, PrEP users who have sex with men living with HIV may feel more confident to rely on the protective effect of PrEP. The decreased condom use could further indicate an increase in sexual comfort.

Our results dovetail nicely with findings regarding the impact of other forms of bio-medical HIV prevention. Notably, treatment as prevention (TasP) has also been shown to play a role in reducing fear and HIV-related stigma (Persson, 2016). So far, awareness and acceptability of TasP has however been limited (Card et al., 2018; Young \& McDaid, 2014), despite the strong evidence of its effectiveness (Cohen, 2019; Rodger et al., 2019). In recent years, TasP 


\section{Chapter 6}

has become more prominent in health promotion campaigns using the slogan "Undetectable is Untransmittable", aiming to increase awareness of TasP among MSM (Eisinger et al., 2019; Rendina \& Parsons, 2018). PrEP and TasP may complement each other (Tester \& Hoxmeier, 2020), as it is suggested that PrEP users have greater knowledge of TasP and greater willingness to rely on TasP (Holt, Draper, et al., 2018). The emergence of PrEP and TasP adds new possibilities for partner selection among MSM who want to mitigate their HIV risk. Instead of selecting a sex partner based on HIV-status (serosorting), MSM can now select partners based on PrEP use ('PrEP sorting'; Martinez \& Jonas, 2019; Wang et al., 2020) or viral load ('biomed matching'; Grov et al., 2018; Newcomb et al., 2016). Both TasP and PrEP can contribute to a decrease in HIV stigma and more comfort during with sex with men living with HIV.

This study has several strengths and limitations. This study is one of the first to investigated changes regarding feelings of fear and comfort related to having sex with HIV-positive men among PrEP users using a quantitative, longitudinal design covering the first 6 months of PrEP use. Moreover, we investigated this in a Dutch context with mostly informal PrEP users, while earlier similar studies were conducted in Australia and the United States where the formal availability of PrEP is much higher (Keen et al., 2020; Whitfield et al., 2018). At the time of our study, formal PrEP services were not implemented yet, and early PrEP adopters therefore had to rely on community information regarding accessing and using PrEP. A limitation is that participants' behaviors and attitudes are assessed by self-report and may be susceptible to social desirability bias. Another limitation is that we did not assess attitudes regarding sex with men living with HIV of PrEP users who indicated not having sex with men living with HIV, so we could not compare attitudes. Also, we did not ask about motives why some PrEP users did not have sex with sex partners living with HIV. This could be a direction for future research, for example to investigate whether this relates to HIV-stigma or to a lack of trust in biomedical protection (i.e. PrEP and TasP). A further limitation is that our sample was recruited via convenience sampling, limiting the representativeness of the sample. We recruited participants via the PrEP advocacy website PrEPnu.nl. Because this website provides information about using PrEP and obtaining PrEP in a context of limited availability, our sample may have an overrepresentation of PrEP users who are well-informed and are more engaged in sexual health. It is possible that these MSM are also more likely to have sex with men living with HIV for other reasons. We could not rule out that other characteristics of these PrEP users are contributing to their attitudes regarding sex with men living with HIV, next to their PrEP use. Put differently, PrEP use could be a proxy for these underlying characteristics. A 
final limitation is that we only analyzed the data of participants who indicated to have sex with men living with HIV at both points of assessment (T1 and T2) to be able make a comparison between these two measurements. However, consequently, participants who indicated to have sex with men living with HIV at T1 but not at T2 $(n=7)$ and vice versa $(n=17)$ were not included in the analysis, as they only answered the items at one point of assessment.

To conclude, our findings suggest that PrEP helps to reduce fear of HIV and to increase comfort having sex with sex partners living with HIV. Over a rather short period of time (3-6 months after the onset of PrEP use), PrEP users also felt safer to not use condoms when having sex with sex men living with HIV. Taken together, the findings suggest that PrEP use may contribute to decreasing HIV-related stigma and to improve sexual interactions between serodiscordant MSM. Together with earlier findings, our results provide evidence for the psycho-social and behavioral potential of PrEP use on closing the serodivide. 


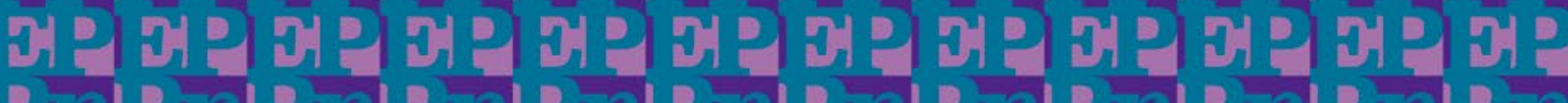
$\mathrm{D}$
$\mathrm{C}$

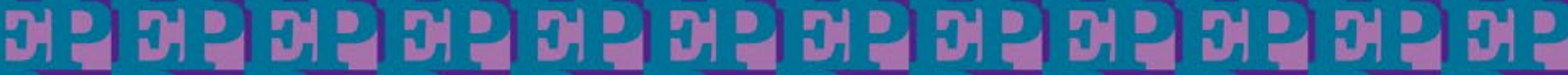
D.

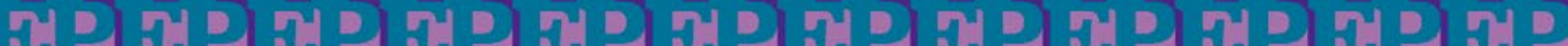
in -

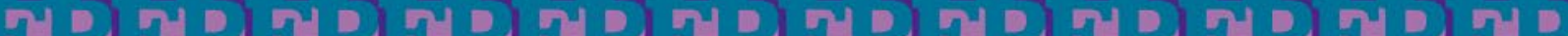
ick

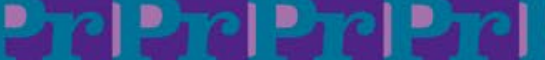

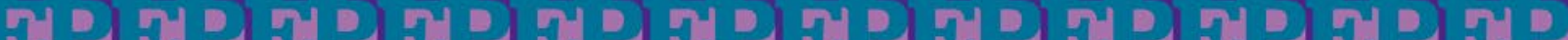
ति

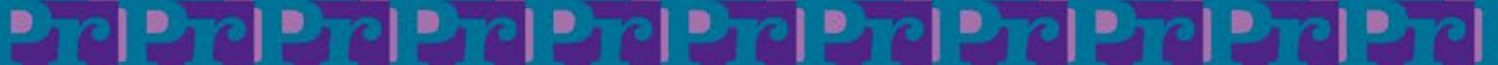

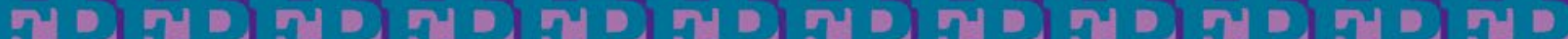
ick

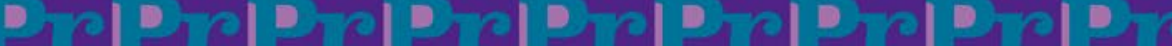

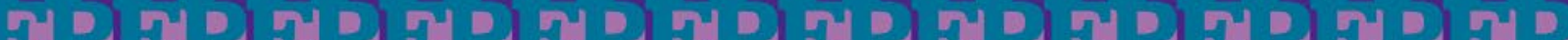

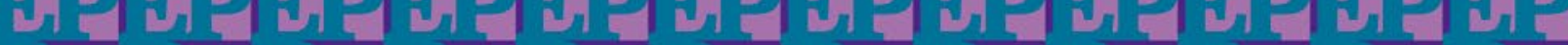

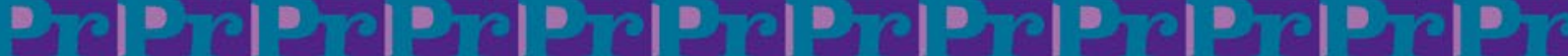

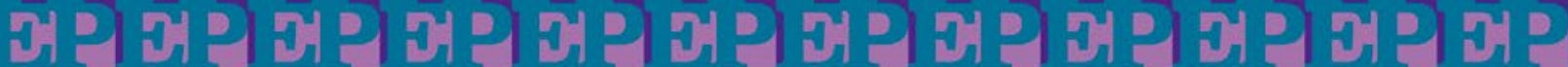
D

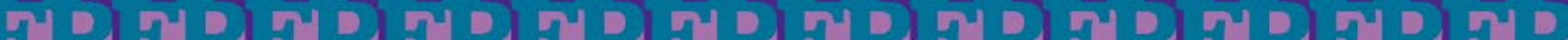
il

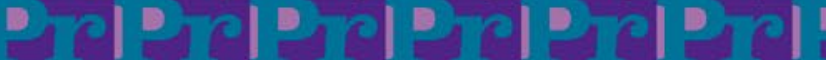

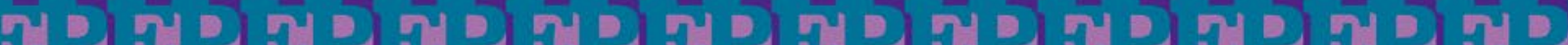
I

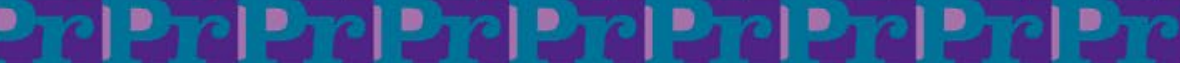

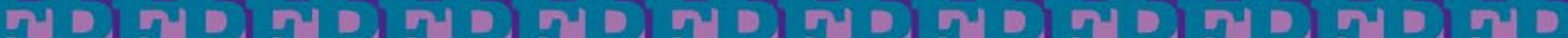
15

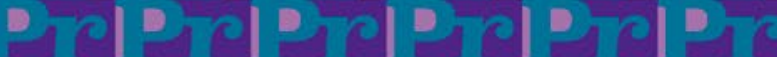

D 10

$\begin{array}{lll}4 & \mathrm{D}\end{array}$

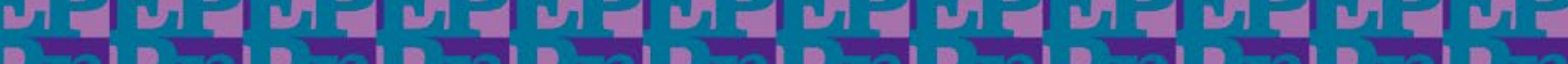
D.

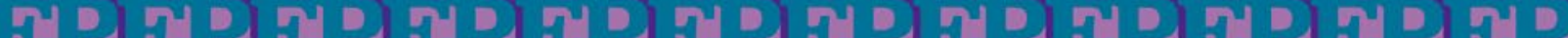
D

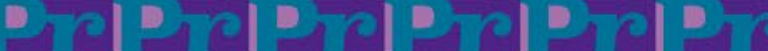

Fل N Q

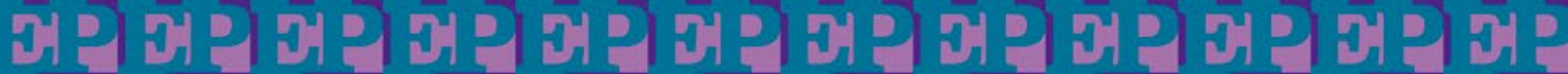

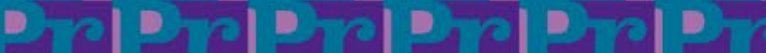

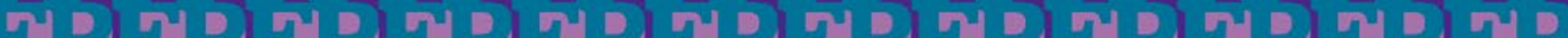

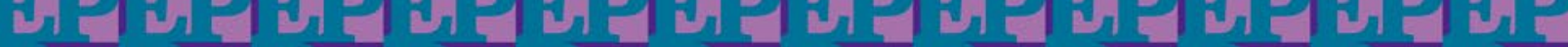

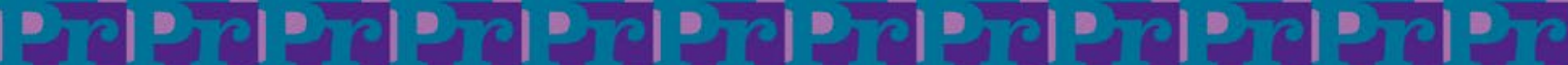

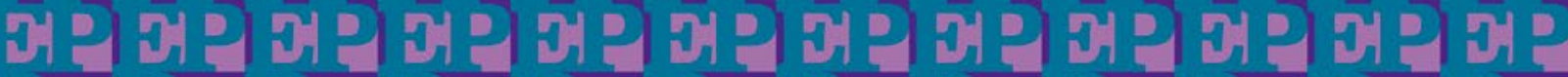


CHAPTER 7

General discussion 


\section{Chapter 7}

In this general discussion, I will discuss the main findings regarding the aims of this thesis. This thesis aimed to answer the following four research questions:

1. What are the attitudes and usage intentions of MSM for PrEP in the Netherlands?

2. How do high-risk MSM in the Netherlands obtain PrEP and access PrEP-related healthcare?

3. What are the hurdles for PrEP use in the Netherlands?

4. What is the sexual behavior of PrEP users after PrEP initiation?

After describing the main findings, the implications of these findings for the societal and scientific debates around PrEP are discussed. Subsequently, the strengths and limitations of this thesis are discussed. Finally, the directions for future research and the conclusions are discussed.

\section{Main findings}

First, we examined the attitudes towards PrEP and interest in PrEP in the study described in chapter 2. We conducted this study in the summer of 2016, before the European Medicines Agency (EMA) approved PrEP and before PrEP was available in the Netherlands. At that time, many MSM were already aware of PrEP: $89.9 \%$ of the participants had heard of PrEP and $85.4 \%$ of them had correct knowledge of PrEP. About half of the participants were interested in PrEP and had the intention to start using PrEP when it would become available. Factors associated with interest in PrEP were being single, having correct prior PrEP knowledge, not having used a condom the last time when having sex, and having ever used drugs in a sexual context. This suggests that MSM who may benefit from PrEP (i.e. those with highrisk behavior) are indeed those who are more interested in PrEP. In addition, we found that the recruitment strategy was also significantly related to interest in PrEP: Participants of the research panel AmsterdamPinkPanel were less interested in PrEP compared to participants recruited via convenience sampling. Other studies also demonstrated differences in interest in PrEP when participants are recruited in various ways, for example in STI clinics or online (Beymer et al., 2018; Ferrer et al., 2016). This indicates that it is important to take recruitment strategies and sample characteristics into account in explaining study findings.

For the second research question, we examined how MSM in the Netherlands obtain PrEP and access PrEP-related healthcare. Although PrEP was not formally available in the Netherlands in 2016, 6.6\% of the participants in the study described in Chapter 2 already used PrEP. This indicated that MSM are obtaining PrEP through informal channels. In the study 
in chapter 3 we qualitatively explored the ways in which PrEP users obtained PrEP and what their experiences were regarding accessing PrEP. This study was conducted in the beginning of 2018, when PrEP was available for an affordable price ( $€ 50$ per 30 pills) in the Netherlands. Participants who first bought PrEP informally abroad or online, had mostly switched to buying PrEP formally at a pharmacy in the Netherlands. Still, there were some PrEP users who remained procuring PrEP via informal channels, because they found it to be more convenient or cheaper. In addition to access to PrEP, we also explored access to PrEP-related healthcare. Some participants indicated that they had to switch general practitioners (GP) because their previous GP had moralistic ideas about sex and PrEP and therefore did not want to prescribe PrEP. According to the participants, PrEP knowledge was limited among GPs, but they were willing to be informed by PrEP users. Renal function testing was usually conducted via the GP, but not all PrEP users found it necessary to do this regularly. Sexually transmitted infection (STI) testing was usually conducted in the public health centers, but waiting lists discouraged some PrEP users to test every three months as is recommended in the national PrEP guidelines. Overall, informal PrEP users were quite capable of arranging PrEP and the care associated with PrEP for themselves.

The third research question was to identify barriers for PrEP use. Since it was assumed that PrEP-related stigma could be a barrier for PrEP use (Calabrese \& Underhill, 2015), we explored the topic of stigma in the qualitative study in chapter 3. PrEP-related stigma seemed to be limited. Most PrEP users received positive responses to their PrEP usage. For example, they were seen as a favorable sex partner, because other MSM assumed that PrEP users wanted to have sex without a condom. However, stigmatizing reactions also emerged. For example, PrEP users were rejected for sex because other MSM thought that PrEP users would have STIs due to irresponsible sexual behavior. In the study in chapter 4 we examined correlates, and potential barriers, for PrEP uptake in more detail. We found that PrEP uptake was higher after the price drop of PrEP, among MSM who were in a favorable financial situation, and among MSM who had previously had a post-exposure prophylaxis (PEP) treatment. The price of PrEP is thus an important barrier for PrEP uptake, and it was the most common barrier for PrEP uptake in a report of 32 other European and Asian countries (Hayes et al., 2019). Our study showed that the price of PrEP mainly played a role in the PrEP uptake among MSM in an average financial situation. MSM with a low financial status still could not afford to pay PrEP despite the price drop, while MSM with a high financial status could afford PrEP regardless of the price. 


\section{Chapter 7}

The fourth research question was to investigate the sexual behavior of PrEP users in the Netherlands. This subject was addressed in multiple studies. We explored the sexual behavior of PrEP users in the qualitative study in chapter 3. PrEP users described that their condom use had generally decreased since they started using PrEP. However, they still used condoms in certain situations, depending on the perceived risk and their familiarity with the sex partner. An important benefit of PrEP use was a reduced fear of HIV. PrEP users felt more in control of their sex life and experienced more sexual freedom, which made them more willing to experiment with sex practices they did not dare before. In the study in chapter 5 we investigated the psychosocial benefits of PrEP quantitatively. PrEP users reported consistent improvements in quality of sex life after PrEP initiation. Reduced fear for HIV was related to improved quality of sex life. In contrast to our expectations, this association was not mediated by condom use. Drug use in a sexual context (chemsex) was high in this group, but PrEP users reported no increase in experimentation with drugs not previously used since PrEP initiation.

In the study in chapter 6 we investigated more in detail the attitudes of PrEP users when having sex with men living with HIV. The majority of PrEP users indicated to have sex with men living with HIV. They reported high levels of reduced fear for HIV and increased comfort when having sex with men living with HIV. They also indicated that since their PrEP use they felt safer to have condomless sex with men living with HIV. These feelings increased over time and showed that PrEP users may have been more confident relying on PrEP over time. The findings suggest that PrEP can play a role in overcoming the serodivide, and that this manifests quite soon after the onset of PrEP use.

\section{Implications}

Below I describe the implications of the findings, first for the Dutch context, then in the context of other countries with a similar PrEP situation, and finally the implications for the promotion of PrEP internationally.

\section{Implications for PrEP use in the Netherlands}

A first implication is that we notice that there is a gap between interest in PrEP and actual uptake of PrEP among MSM in the Netherlands. In the study in chapter 2 we found that the interest in PrEP, and the intention to start using PrEP is quite high. Interest in PrEP was related to being single, having correctly prior PrEP knowledge, not having used a condom the last time when having sex, and having ever used drugs in a sexual context. These are exactly the men who can benefit from PrEP. Nevertheless, we found in chapter 4, in a study under 
MSM with a high interest in PrEP, that ultimately the price of PrEP and the financial situation of the participants is significantly related to actual PrEP uptake. Sexual risk behaviors were not significant predictors of PrEP uptake, while they were related to interest in PrEP. This demonstrates that PrEP mainly reaches those MSM who can afford it, even though there is high interest from other MSM with high-risk behaviors. In order to increase the impact of PrEP on the reduction of new HIV infections, it is important that PrEP is used as much as possible by MSM who are eligible for PrEP. The price of PrEP should not be a deciding factor in the use of PrEP. PrEP uptake can increase if the price is at the lowest possible level. At the time of our research PrEP was available for $€ 50$ per 30 pills (AidsFonds, 2018). PrEP is now available through the public health centers and is partially reimbursed through the national health insurance scheme (Ministry of Health Welfare and Sport, 2019). This has made PrEP more accessible for a larger group of MSM. Nevertheless, it remains important to focus on MSM in less favorable (financial) positions; the price of PrEP can still be a barrier for them, while they may be at increased risk of HIV.

Secondly, this thesis shows that informal PrEP use exists, and may continue to exist. Studies on informal PrEP use are scarce, and our research is the first study investigating informal PrEP use in the Netherlands. Most of the informal PrEP users switched to formal PrEP use as soon as PrEP became more affordable in the Netherlands. However, some PrEP users continued informal PrEP procurement. Continued informal PrEP use was also found in a study in Germany, in which $17.4 \%$ of the 2000 PrEP users obtained PrEP informally, despite the formal availability of generic PrEP in Germany (Koppe et al., 2019). A possible explanation is that the price of generic PrEP is still not affordable for some MSM. In the study in chapter 3 , we also found that some participants continued informal PrEP procurement out of convenience, since they received PrEP through friends who bought PrEP abroad. Most informal PrEP users were well informed about PrEP, and therefore used PrEP correctly and followed the guidelines for regular testing. However, there were also some informal PrEP users who did not get tested regularly, particularly regarding renal function testing. They considered this not necessary or they did not know where to get tested. It is important that also informal PrEP users have barrier-free access to HIV, STI, and renal function testing to enable them to follow the guidelines for safe PrEP use. In the Netherlands, the Amsterdam Municipal Health Service (GGD) already organized consultations for the guidance of 250 informal PrEP users over a period of 24 months (GGD Amsterdam, 2017). 


\section{Chapter 7}

Implications for PrEP use in other countries with informal PrEP use

It is remarkable that studies on informal PrEP use are so scarce, as informal PrEP use occurs in many countries in Europe. The findings of the studies in this thesis on informal PrEP use are therefore not only relevant for the Netherlands, but also for other countries, in particular these where PrEP is not yet formally available. A report of the European Centre for Disease Prevention and Control (ECDC, 2019) shows that in 2017 in some countries informal PrEP use was the most common form of PrEP procurement. For example, in Switzerland 67\% of the PrEP users obtained PrEP informally, and in the UK 59\%. These figures are likely to have changed by now as the availability and affordability of PrEP has increased in a number of countries. However, in 2019 there were still 37 out of 53 reporting countries in Europe and neighboring countries that had not implemented formal access to PrEP (European Centre for Disease Prevention and Control, 2019c). As long as PrEP is not formally available, PrEP is likely used informally in these countries. For example, in countries in Eastern Europe and Central Asia nongovernmental organizations (NGOs) and community organizations are actively promoting PrEP and making PrEP (informally) available (Begovac et al., 2019; Zardiashvili, 2019). The studies in this thesis show that this informal use of PrEP can work quite well, but it may mainly reach MSM who have a high community engagement, and who are consequently well informed about PrEP. For them, the transition to formal PrEP likely proceeds smoothly as soon as formal PrEP is accessible and affordable.

\section{Implications for PrEP use at a global level}

Furthermore, the studies in this thesis have implications for PrEP use on a global level, which are not limited to a Dutch or European context. The studies in this thesis provide insight into the role of PrEP in relation to other HIV prevention strategies. In the media PrEP was sometimes presented as an alternative to condoms, and the use of terms such as 'PrEP whores' created the impression that PrEP was used to facilitate frequent condomless anal sex (Spieldenner, 2016). This gives a distorted depiction of PrEP. Although we indeed observed in the studies in chapter 5 and 6 that PrEP users had a decreased condom use after PrEP initiation, we also observed in the qualitative study in chapter 3 that PrEP users often make an appraisal per situation whether they still use a condom, depending on the perceived risk. This demonstrates that PrEP should be seen as part of a range of HIV prevention strategies that can be combined. A latent class analysis in a study from Canada, where PrEP has been formally available for some time, showed that PrEP is often combined with other HIV prevention strategies (Doyle et al., 2020). 
In addition to PrEP and condoms, there are other strategies that are part of the comprehensive framework of HIV prevention that can be combined. The combination of PrEP and Treatment as Prevention (TasP) probably has the most impact in reducing the incidence of new HIV infections (Doyle et al., 2020; Jones et al., 2019; Rozhnova et al., 2018). In practice, TasP facilitates so-called viral load sorting: the selection of sex partner based on whether the person living with HIV is virally suppressed (Grov et al., 2018). This strategy is more than merely selecting a partner on the basis of HIV status, as is the case with serosorting (Eaton et al., 2009). Besides viral load sorting and serosorting, PrEP sorting also occurs, whereby sex partners are selected based on their PrEP use (Martinez \& Jonas, 2019). In the qualitative study in chapter 3, PrEP users indicated that they were sometimes seen as favorable sex partners, because others preferred to have sex with them without a condom (and assumed that PrEP users would have condomless sex): This shows that non-PrEP users relied on the protection of PrEP (of the PrEP user) against HIV and the transmission of HIV. PrEP is hence not an isolated phenomenon: It can be used in combination with other HIV prevention strategies, and it also has an impact on MSM who do not use PrEP.

This thesis also contributes to our understanding of disparities in PrEP uptake. Particularly in the United States, it is often found that certain demographic groups, such as Black, Hispanic, and younger individuals are less likely to use PrEP (Bush et al., 2016; Kamitani et al., 2020; Kelley et al., 2015; Marcus et al., 2016; Rolle et al., 2017; Snowden et al., 2017), while they are at increased risk of HIV (Hess et al., 2017). Likewise, in the Netherlands non-Western migrants have an increased risk of HIV (Visser et al., 2017), but often have less access to HIV prevention services such as PrEP (Bil et al., 2019). The increased risk of HIV and the lower likelihood of PrEP use among certain demographic groups are not isolated events, and can jointly be understood from a syndemic perspective. The syndemic perspective is a framework for understanding health conditions as resulting from the interplay of the co-occurrence of multiple diseases with social, economic, and environmental factors (Singer et al., 2017; The Lancet, 2017; Tsai et al., 2017). In our studies, we found that economic and environmental factors play a role in PrEP access and PrEP uptake. In the study in chapter 4 we found that MSM in a less favorable financial situation were less likely to use PrEP, despite high interest in PrEP. Due to decreases in the price of PrEP, PrEP will become more available for less financially privileged people, but even with a low price of PrEP it remains important to ensure the availability of PrEP for them. Regarding environmental factors, we found in the qualitative study in chapter 3 that PrEP use in rural areas was lower and much less accepted. Also in rural areas in the United States, it was found that MSM have to travel far to obtain PrEP (Weiss et 


\section{Chapter 7}

al., 2018) and that access to information and PrEP-related healthcare is limited (C. Owens et al., 2020). In France, it was also found that gay men who live in villages, or in areas with few PrEP access points, are less likely to use PrEP (Annequin et al., 2020).

Lastly, the studies in this thesis underline the importance of the role of PrEP in sexual health. Sexual health not only refers to the prevention of STIs, but it is "a state of physical, emotional, mental and social well-being in relation to sexuality" (WHO 2015 sexual health). Both the qualitative study in chapter 3 and the quantitative studies in chapters 5 and 6 showed that PrEP users have less fear of HIV since they started using PrEP and therefore are more likely to enjoy sex. PrEP is not only beneficial for the prevention of HIV infections, but also for improving the quality of sex life. PrEP uptake may increase if the psychosocial benefits of PrEP are promoted. Moreover, the psychosocial benefits of PrEP can contribute to bridging the serodivide: PrEP users may feel more comfortable having sex with men living with HIV and may be more willing to have sex with men living with HIV.

\section{Strengths and limitations}

A strength of this thesis is that it is the first in the Netherlands to study PrEP use outside a clinical setting, such as in the demonstration trial AMPrEP (Public Health Service Amsterdam, 2015). There is a need to study PrEP in a real-world setting, to investigate the social implications of PrEP beyond its clinical efficacy (Auerbach \& Hoppe, 2015). Moreover, clinical trials may give a biased view of changes in sexual behavior, because of the selection of participants (i.e. selected participants are MSM with (extreme) high risk behavior) or because of the included PrEP-related healthcare, such as behavioral counseling and regular HIV and STI testing, that may be absent in a real-world situation (Buttram, 2018; Kurtz \& Buttram, 2016). Therefore, the qualitative study in chapter 3 explored the experiences of PrEP users on a wide range of topics. In addition, some behavioral changes may not be present immediately after PrEP initiation, as a meta-analysis of open-label studies showed that more recent studies and studies with a longer follow-up time did show an increase in STIs among PrEP users (Traeger et al., 2018). Therefore, we included multiple points of assessment in the studies in chapter 4, 5 , and 6 .

Another strength of this thesis is the in-depth analysis of recruitment strategies in the study in chapter 2. We found that MSM participants recruited through the research panel AmsterdamPinkPanel were less interested in PrEP and had a lower intention to use PrEP than MSM participants who were recruited via convenience sampling. These MSM were of older 
age, and their lower interest in PrEP may be explained by their personal history of HIV prevention. Notably, they may be more likely to rely on established HIV prevention strategies that have worked for them for many years, and they may not feel a need to change their HIV prevention strategy to PrEP use. The findings of this study indicate that recruitment strategies have a substantial impact on findings regarding interest in PrEP and intention to use PrEP. We recommended that researchers tailor their recruitment strategies to their research aims and inclusion criteria. For the other studies in this thesis, we used convenience sampling and recruited participants via the website of the Dutch PrEP advocacy group PrEPnu.nl. This allowed us to target potential PrEP users in particular, in a context and time (2017) when PrEP use was limited. As a result, we mainly recruited PrEP users and MSM with a high interest in PrEP. This was useful for the research aims, but it limits the representativeness of the studies to the whole MSM population.

There are several further limitations to the studies in this thesis. Regarding the representativeness of the findings; it is unclear to what extent the findings of our studies are generalizable to future PrEP users. The PrEP users in the studies in this thesis can be seen as early adopters who had to make an increased effort to obtain (informal) PrEP, and who often had high PrEP knowledge because of high community engagement. These early adopters may differ from later PrEP adopters. According to the Diffusion of Innovation theory (Rogers, 2010), the acceptance and dissemination of new ideas and products progresses through acceptance by a sequence of groups within social systems or societies. This theory has also been applied to the uptake of HIV prevention strategies (Bertrand, 2004). The innovators and early adopters, who are the first to embrace a prevention strategy, differ in their characteristics from the early and late majority; they are more open to change and take more risks (Rogers, 2010). In the context of PrEP, a study in Australia found that early PrEP adopters reported higher risk sexual practices (compared to non-users) and were more likely to know other PrEP users (Holt et al., 2019). The authors suggested that, according to the Diffusion of Innovation theory, future PrEP users may require greater reassurance about the efficacy of PrEP to increase PrEP uptake among the early and late majority.

Another limitation of this thesis is that we only studied PrEP interest and use among MSM and not among other populations. While it is relevant to study PrEP use among MSM, MSM are the main risk population for HIV in the Netherlands (Van Sighem et al., 2019), there are other populations that may benefit from PrEP as well. A priority group for PrEP is transgender individuals. A recent meta-analysis found that transgender women are 66 times more 


\section{Chapter 7}

likely to have HIV compared to adults in the general population worldwide (Stutterheim et al., 2020). Transgender women are often recognized in national PrEP guidelines as a risk group for PrEP eligibility, but are often underrepresented in PrEP studies (Hoornenborg, Krakower, et al., 2017). Studies in the United States show that PrEP awareness is low among transgender women (Poteat et al., 2019), and consequently, their PrEP use is also low (Reisner et al., 2019). Transgender men have a lower HIV risk than transgender women, but are still at increased risk of HIV compared to the general population (Stutterheim et al., 2020). A study in the United States found that $24.3 \%$ of transgender men would meet eligibility criteria for PrEP, but only $3 \%$ had been prescribed PrEP (Golub, Fikslin, Starbuck, et al., 2019). Future research should address the HIV prevention needs of transgender individuals to improve PrEP awareness and PrEP use among them.

A final limitation of this research is that not all research aims that were formulated in the original research proposal were investigated, due to the rapidly changing context of PrEP in the Netherlands. One of the aims in the research proposal was targeted at the effect of PrEP use on the behavior of non-users. We expected that PrEP use would be limited in the Netherlands, because PrEP was not approved in the Netherlands at the time of writing the research proposal. Therefore, we expected to have few PrEP users in our study, and we planned to investigate the effect of PrEP on non-users. However, PrEP uptake increased in 2017 and 2018 after generic (and cheaper) PrEP became available, and we decided to focus on the experiences of PrEP users themselves. Moreover, the research proposal focused on MSM attending circuit parties, as we expected that PrEP use would be higher among these MSM. However, as PrEP use became normalized thanks to the formal approval and increased availability, we did not limit our recruitment to MSM attending circuit parties, but we aimed to include a broader group of PrEP using MSM.

\section{Directions for future research}

In the section above, we already mentioned a few directions for future research following the limitations, namely to investigate differences between early PrEP adopters and late PrEP adopters, and to investigate PrEP use among other risk groups such as transgender individuals. In addition, there are a number of other topics that we recommend for future research.

Firstly, there is a need for research with people who stop or have to stop using PrEP. This was not addressed in this thesis because we focused on MSM who started using PrEP as PrEP was a new phenomenon in the Netherlands. A next step is to investigate the sexual health of 
people who stop using PrEP, and in particular what their HIV prevention strategies are when they stop using PrEP. There are several reasons to stop using PrEP: Someone can voluntarily stop with PrEP because they perceive their risk of HIV as lower (e.g. they get into a monogamous relationship). It could also happen that someone has to stop involuntarily because of side effects or reduced access to PrEP due to a move or changes in insurance policies (Jonas \& Yaemim, 2018). People who stop using PrEP can remain at risk of HIV (Spinelli et al., 2020). Tailored HIV prevention strategies are needed for MSM who stop using PrEP, as going back to condoms may not be a viable strategy in all cases (Jonas \& Yaemim, 2018).

Secondly, there is a need for research on how to reach young MSM in the Netherlands. The Dutch PrEP guideline advises PrEP use for MSM who have had condomless anal intercourse, had an STI, or have been prescribed a PEP treatment in the past six months (Hoornenborg \& Rijnders, 2019). This excludes young people who are not yet sexually active, but want to become so, from the eligibility criteria, because the criteria are based on sexual behaviors that inexperienced young people did not yet engage in. Nevertheless, PrEP can be prescribed to them, as the guideline also encourages PrEP to be prescribed to those who ask for it. A number of studies have already been done in the United States regarding PrEP and adolescents. A study among 1541 adolescent sexual minority males aged 13 - 18 years found that only $28 \%$ were aware of PrEP (Dunville et al., 2020). Other studies found a higher awareness of PrEP among adolescents and investigated barriers for PrEP uptake, such as the role of parents (Macapagal et al., 2020; Moskowitz et al., 2020). Some young MSM were afraid that their parents would be unsupportive of their PrEP use, which limited their interest in PrEP (Moskowitz et al., 2020).

Lastly, it is important that future research continues to investigate informal PrEP use. Informal PrEP use exists in many countries, and may emerge as long as formal PrEP access is not implemented. In addition, informal PrEP can re-emerge in countries where PrEP is already formally available when new forms of PrEP are introduced. Currently tenofovir disoproxil fumarate with emtricitabine (TDF-FTC, Truvada) is used for PrEP in most countries, but in the United States, tenofovir alafenamide with emtricitabine (TAF-FTC, Descovy) has been approved for PrEP by the Food and Drug Administration (FDA) since October 2019 (U.S. Food and Drug Administration, 2019). TAF-FTC may have fewer side effects than TDF-FTC, but it is more expensive (Krakower et al., 2020). The higher price of TAF-FTC may motivate MSM to obtain this form of PrEP outside formal channels for a lower price. Furthermore, long-acting injectable PrEP may become available in the coming years, as initial studies show 
that cabotegravir is a highly effective form of long-acting injectable PrEP (Cohen, 2020). The introduction of long-acting injectable PrEP may lead to a new wave of informal PrEP use if this form of injectable PrEP is first only available in a limited number of countries, triggering interested MSM to travel to these countries for PrEP. It is likely that only a small amount of people will opt for informal PrEP use, yet it is important to monitor whether they have additional risks due to possible limited medical supervision.

\section{Conclusion}

The studies in this thesis examined the interest and attitudes of MSM towards PrEP and the experiences of PrEP users during the early years of PrEP in the Netherlands. Informal PrEP users had a high level of PrEP knowledge through community engagement. Most informal PrEP users switched to formal PrEP procurement, as soon as generic PrEP became available in the Netherlands for a more affordable price. PrEP-related healthcare should remain accessible for informal PrEP users, as informal PrEP use may continue to exist when new forms of PrEP appear on the market. MSM with high-risk sexual behaviors were interested in PrEP, but PrEP use mostly happened among MSM who could afford it. The studies in this thesis also demonstrate that the benefits of PrEP go beyond just HIV prevention: PrEP has a positive impact on the quality of sex life by reducing fear for HIV. 
General discussion 
REFERENCES 
Achterbergh, R. C. A., Hoornenborg, E., Boyd, A., Coyer, L., Meuzelaar, S. J. A., Hogewoning, A. A., Davidovich, U., van Rooijen, M. S., Schim van der Loeff, M. F., Prins, M., \& de Vries, H. J. C. (2020). Changes in mental health and drug use among men who have sex with men using daily and event-driven pre-exposure prophylaxis: Results from a prospective demonstration project in Amsterdam, the Netherlands. EClinicalMedicine. https://doi.org/10.1016/j.eclinm.2020.100505

Aghaizu, A., Mercey, D., Copas, A., Johnson, A. M., Hart, G., \& Nardone, A. (2013). Who would use PrEP? Factors associated with intention to use among MSM in London: A community survey. Sexually Transmitted Infections, 89(3), 207-211. https://doi.org/ doi:10.1136/sextrans-2012-050648

Ai, C., \& Norton, E. C. (2003). Interaction terms in logit and probit models. Economics Letters, 80(1), 123-129. https://doi.org/10.1016/S0165-1765(03)00032-6

AIDES. (2016). First results of the Flash!PrEP in Europe online survey. https://www.aides.org/ FlashPrEPinEurope

AidsFonds. (2018). PrEP nu voor 50 euro beschikbaar bij alle apotheken. https://aidsfonds.nl/ nieuws/prep-nu-voor-50-euro-beschikbaar-bij-alle-apotheken/

Almeda, J., Casabona, J., Simon, B., Gerard, M., Rey, D., Puro, V., \& Thomas, T. (2004). Proposed recommendations for the management of HIV post-exposure prophylaxis after sexual, injecting drug or other exposures in Europe. Eurosurveillance, 9(6), 35-40.

Annequin, M., Villes, V., Delabre, R. M., Alain, T., Morel, S., Michels, D., Schmidt, A. J., Velter, A., \& Rojas Castro, D. (2020). Are PrEP services in France reaching all those exposed to HIV who want to take PrEP? MSM respondents who are eligible but not using PrEP (EMIS 2017). AIDS Care, 0(0), 1-10. https://doi.org/10.1080/09540121.2020.1739219

Arnold, T., Brinkley-Rubinstein, L., Chan, P. A., Perez-Brumer, A., Bologna, E. S., Beauchamps, L., Johnson, K., Mena, L., \& Nunn, A. (2017). Social, structural, behavioral and clinical factors influencing retention in Pre-Exposure Prophylaxis (PrEP) care in Mississippi. PLoS ONE, 12(2), e0172354. https://doi.org/10.1371/journal.pone.0172354

ATLAS.ti Scientific Software Development GmbH. (2018). ATLAS.ti (Version 8.3.0). https:// atlasti.com

Auerbach, J. D., \& Hoppe, T. A. (2015). Beyond "getting drugs into bodies": social science perspectives on pre-exposure prophylaxis for HIV. Journal of the International AIDS Society, 18(4 (Suppl 3)). https://doi.org/10.7448/IAS.18.4.19983

AVAC. (2020). PrEP Watch. https://www.prepwatch.org/

Ayling, R., \& Mewse, A. J. (2009). Evaluating internet interviews with gay men. Qualitative Health Research, 19(4), 566-576. https://doi.org/10.1177/1049732309332121 
Baeten, J. M., Donnell, D., Ndase, P., Mugo, N. R., Campbell, J. D., Wangisi, J., Tappero, J. W., Bukusi, E. A., Cohen, C. R., Katabira, E., Ronald, A., Tumwesigye, E., Were, E., Fife, K. H., Kiarie, J., Farquhar, C., John-Stewart, G., Kakia, A., Odoyo, J., ... Celum, C. (2012). Antiretroviral prophylaxis for HIV prevention in heterosexual men and women. New England Journal of Medicine, 367(5), 399-410. https://doi.org/10.1056/NEJMoa1108524

Barash, E. A., \& Golden, M. (2010). Awareness and use of HIV pre-exposure prophylaxis among attendees of a Seattle gay pride event and sexually transmitted disease clinic. AIDS Patient Care and STDs, 24(11), 689-691. https://doi.org/10.1089/apc.2010.0173

Bauermeister, J., Meanley, S., Pingel, E., Soler, J., \& Harper, G. (2014). PrEP awareness and perceived barriers among single young men who have sex with men. Current HIV Research, 11(7), 520-527. https://doi.org/10.2174/1570162X12666140129100411

Begovac, J., Skrzat-Klapaczynska, A., \& Kowalska, J. (2019). Where are we with PrEP use in Central and Eastern Europe?-data from the ECEE Network Group. Hiv Medicine, 20(Suppl. 9), 242.

Bertrand, J. T. (2004). Diffusion of innovations and HIV/AIDS. Journal of Health Communication, 9(sup1), 113-121. https://doi.org/10.1080/10810730490271575

Beymer, M. R., Holloway, I. W., \& Grov, C. (2018). Comparing self-reported demographic and sexual behavioral factors among men who have sex with men recruited through Mechanical Turk, Qualtrics, and a HIV/STI clinic-based sample: Implications for researchers and providers. Archives of Sexual Behavior, 47(1), 133-142. https://doi.org/10.1007/s10508016-0932-y

Beyrer, C., Baral, S. D., van Griensven, F., Goodreau, S. M., Chariyalertsak, S., Wirtz, A. L., \& Brookmeyer, R. (2012). Global epidemiology of HIV infection in men who have sex with men. The Lancet, 380(9839), 367-377. https://doi.org/10.1016/S0140-6736(12)60821-6

Beyrer, C., Sullivan, P., Sanchez, J., Baral, S. D., Collins, C., Wirtz, A. L., Altman, D., Trapence, G., \& Mayer, K. (2013). The increase in global HIV epidemics in MSM. AIDS, 27(17), 2665-2678. https://doi.org/10.1097/01.aids.0000432449.30239.fe

Biello, K. B., Oldenburg, C. E., Mitty, J. A., Closson, E. F., Mayer, K. H., Safren, S. A., \& Mimiaga, M. J. (2017). The "safe sex" conundrum: Anticipated stigma from sexual partners as a barrier to PrEP use among substance using MSM engaging in transactional sex. AIDS and Behavior, 21(1), 300-306. https://doi.org/10.1007/s10461-016-1466-y

Bil, J. P., Davidovich, U., van der Veldt, W. M., Prins, M., de Vries, H. J. C., Sonder, G. J. B., \& Stolte, I. G. (2015). What do Dutch MSM think of preexposure prophylaxis to prevent HIV-infection? A cross-sectional study: AIDS, 29(8), 955-964. https://doi.org/10.1097/ QAD.0000000000000639 
Bil, J. P., Zuure, F. R., Alvarez-del Arco, D., Prins, J. M., Brinkman, K., Leyten, E., van Sighem, A., Burns, F., \& Prins, M. (2019). Disparities in access to and use of HIV-related health services in the Netherlands by migrant status and sexual orientation: a cross-sectional study among people recently diagnosed with HIV infection. BMC Infectious Diseases, 19(1), 906. https://doi.org/10.1186/s12879-019-4477-2

Blashill, A. J., Brady, J. P., Rooney, B. M., Rodriguez-Diaz, C. E., Horvath, K. J., Blumenthal, J., Morris, S., Moore, D. J., \& Safren, S. A. (2020). Syndemics and the PrEP cascade: Results from a sample of young Latino men who have sex with men. Archives of Sexual Behavior, 49(1), 125-135. https://doi.org/10.1007/s10508-019-01470-7

Blumenthal, J., Jain, S., Mulvihill, E., Sun, S., Hanashiro, M., Ellorin, E., Graber, S., Haubrich, R., \& Morris, S. (2019). Perceived versus calculated HIV risk: Implications for pre-exposure prophylaxis uptake in a randomized trial of men who have sex with men. Journal of Acquired Immune Deficiency Syndromes, 80(2), e23-e29. https://doi.org/10.1097/ QAI.0000000000001888

Bourne, A., Alba, B., Garner, A., Spiteri, G., Pharris, A., \& Noori, T. (2019). Use of, and likelihood of using, HIV pre-exposure prophylaxis among men who have sex with men in Europe and Central Asia: Findings from a 2017 large geosocial networking application survey. Sexually Transmitted Infections, 95(3), 187-192. https://doi.org/10.1136/sextrans-2018-053705

Bourne, A., Reid, D., Hickson, F., Torres-Rueda, S., \& Weatherburn, P. (2015). Illicit drug use in sexual settings ('chemsex') and HIV/STI transmission risk behaviour among gay men in South London: findings from a qualitative study. Sexually Transmitted Infections, 91(8), 564-568. https://doi.org/doi:10.1136/sextrans-2015-052052

Brisson, J. (2018). Ethical public health issues for the use of informal PrEP. Global Public Health, 13(10), 1382-1387. https://doi.org/10.1080/17441692.2017.1373139

Brisson, J., \& Nguyen, V. K. (2017). Science, technology, power and sex: PrEP and HIV-positive gay men in Paris. Culture, Health and Sexuality, 19(10), 1066-1077. https://doi.org/1 $0.1080 / 13691058.2017 .1291994$

Brisson, J., Ravitsky, V., \& Williams-Jones, B. (2019). Informal PrEP: an emerging need for nomenclature. The Lancet Public Health, 4(2), e83. https://doi.org/10.1016/S24682667(18)30254-8

Brooks, R. A., Kaplan, R. L., Lieber, E., Landovitz, R. J., Lee, S.-J., \& Leibowitz, A. A. (2011). Motivators, concerns, and barriers to adoption of preexposure prophylaxis for HIV prevention among gay and bisexual men in HIV-serodiscordant male relationships. AIDS Care, 23(9), 1136-1145. https://doi.org/10.1080/09540121.2011.554528 
Brooks, R. A., Landrian, A., Nieto, O., \& Fehrenbacher, A. (2019). Experiences of anticipated and enacted pre-exposure prophylaxis (PrEP) stigma among Latino MSM in Los Angeles. AIDS and Behavior, 23(7), 1964-1973. https://doi.org/10.1007/s10461-019-02397-9

Bush, S., Magnuson, D., Rawlings, M. K., Hawkins, T., McCallister, S., \& Mera Giler, R. (2016). Racial characteristics of FTC/TDF for pre-exposure prophylaxis (PrEP) users in the US. ASM Microbe/ICAAC, 16, 16.

Buttram, M. E. (2018). The informal use of antiretroviral medications for HIV prevention by men who have sex with men in South Florida: initiation, use practices, medications and motivations. Culture, Health \& Sexuality, 20(11), 1185-1198. https://doi.org/10.1080/13 691058.2017.1421709

Buttram, M. E., \& Kurtz, S. P. (2017). Preliminary evidence of HIV seroconversion among HIV-negative men who have sex with men taking non-prescribed antiretroviral medication for HIV prevention in Miami, Florida, USA. Sexual Health, 14(2), 193. https://doi. org/10.1071/SH16108

Cahill, S., Taylor, S. W., Elsesser, S. A., Mena, L., Hickson, D., \& Mayer, K. H. (2017). Stigma, medical mistrust, and perceived racism may affect PrEP awareness and uptake in black compared to white gay and bisexual men in Jackson, Mississippi and Boston, Massachusetts. AIDS Care, 1-8. https://doi.org/10.1080/09540121.2017.1300633

Calabrese, S. K., Earnshaw, V. A., Underhill, K., Krakower, D. S., Magnus, M., Hansen, N. B., Mayer, K. H., Betancourt, J. R., Kershaw, T. S., \& Dovidio, J. F. (2018). Prevention paradox: Medical students are less inclined to prescribe HIV pre-exposure prophylaxis for patients in highest need. Journal of the International AIDS Society, 21(6), 1-8. https://doi. org/10.1002/jia2.25147

Calabrese, S. K., \& Underhill, K. (2015). How stigma surrounding the use of HIV preexposure prophylaxis undermines prevention and pleasure: A call to destigmatize "Truvada Whores.” American Journal of Public Health, 105(10), 1960-1964. https://doi.org/10.2105/ AJPH.2015.302816

Carballo-Diéguez, A., Ventuneac, A., Dowsett, G. W., Balan, I., Bauermeister, J., Remien, R. H., Dolezal, C., Giguere, R., \& Mabragaña, M. (2011). Sexual pleasure and intimacy among men who engage in "bareback sex." AIDS and Behavior, 15(S1), 57-65. https://doi. org/10.1007/s10461-011-9900-7

Card, K. G., Armstrong, H. L., Lachowsky, N. J., Cui, Z., Sereda, P., Carter, A., Montaner, J. S. G., Hogg, R. S., Roth, E. A., \& Moore, D. M. (2018). Belief in treatment as prevention and its relationship to HIV status and behavioral risk. JAIDS Journal of Acquired Immune Deficiency Syndromes, 77(1), 8-16. https://doi.org/10.1097/QAI.0000000000001557 
CDC. (2017). Preexposure prophylaxis for the prevention of HIV infection in the United States - 2017 update: a clinical practice guideline. In Preexposure prophylaxis for the prevention of HIV infection in the United States - 2017 update: a clinical practice guideline. https://stacks.cdc.gov/view/cdc/53509

CDC. (2020). PrEP \& HIV Basics. https://www.cdc.gov/hiv/basics/prep.html

Charpentier, N., Brancourt, M., Sordet, F., \& Laforgerie, J. F. (2014). Nouvelle prévention: Sommes-nous PrEP? Remaides, 90, 39-42.

Closson, E. F., Mitty, J. A., Malone, J., Mayer, K. H., \& Mimiaga, M. J. (2018). Exploring strategies for PrEP adherence and dosing preferences in the context of sexualized recreational drug use among MSM: a qualitative study. AIDS Care, 30(2), 191-198. https://doi.org/10 $.1080 / 09540121.2017 .1360992$

Coelho, L. E., Torres, T. S., Veloso, V. G., Landovitz, R. J., \& Grinsztejn, B. (2019). Pre-exposure prophylaxis 2.0: new drugs and technologies in the pipeline. The Lancet HIV, 6(11), e788-e799. https://doi.org/10.1016/S2352-3018(19)30238-3

Cohen, J. (2020). Long-acting injectable drug prevents HIV infections. Science. https://doi. org/10.1126/science.abc8634

Cohen, M. S. (2019). Successful treatment of HIV eliminates sexual transmission. The Lancet, 393(10189), 2366-2367. https://doi.org/10.1016/S0140-6736(19)30701-9

Collins, S. P., McMahan, V. M., \& Stekler, J. D. (2017). The impact of HIV pre-exposure prophylaxis (PrEP) use on the sexual health of men who have sex with men: A qualitative study in Seattle, WA. International Journal of Sexual Health, 29(1), 55-68. https://doi.org/10.10 80/19317611.2016.1206051

Courtenay-Quirk, C., Wolitski, R. J., Parsons, J. T., \& Gómez, C. A. (2006). Is HIV/AIDS stigma dividing the gay community? Perceptions of HIV-positive men who have sex with men. AIDS Education and Prevention, 18(1), 56-67. https://doi.org/10.1521/aeap.2006.18.1.56

Da Silva-Brandao, R. R., \& Ianni, A. M. Z. (2020). Sexual desire and pleasure in the context of the HIV pre-exposure prophylaxis (PrEP). Sexualities, 23(8), 1400-1416. https://doi. org/10.1177/1363460720939047

Davis, M., Bolding, G., Hart, G., Sherr, L., \& Elford, J. (2004). Reflecting on the experience of interviewing online: Perspectives from the Internet and HIV study in London. AIDS Care, 16(8), 944-952. https://doi.org/10.1080/09540120412331292499

Davis, M., Hart, G., Bolding, G., Sherr, L., \& Elford, J. (2006). Sex and the internet: Gay men, risk reduction and serostatus. Culture, Health and Sexuality, 8(2), 161-174. https://doi. org/10.1080/13691050500526126

Dehlin, J. M., Stillwagon, R., Pickett, J., Keene, L., \& Schneider, J. A. (2019). \#PrEP4Love: An 
evaluation of a sex-positive HIV prevention campaign. JMIR Public Health and Surveillance, 5(2), e12822. https://doi.org/10.2196/12822

Dimitrov, D., Boily, M.-C., Mâsse, B. R., \& Brown, E. R. (2013). Impact of pill sharing on drug resistance due to a wide-scale oral PrEP intervention in generalized epidemics. Journal of AIDS \& Clinical Research, 01(S5). https://doi.org/10.4172/2155-6113.S5-004

Dodds, J. P., Mercer, C. H., Mercey, D. E., Copas, A. J., \& Johnson, A. M. (2006). Men who have sex with men: A comparison of a probability sample survey and a community based study. Sexually Transmitted Infections, 82(1), 86-87. https://doi.org/10.1136/sti.2005.015248

Dowson, L., Kober, C., Perry, N., Fisher, M., \& Richardson, D. (2012). Why some MSM present late for HIV testing: a qualitative analysis. AIDS Care, 24(2), 204-209. https://doi.org /10.1080/09540121.2011.597711

Doyle, C. M., Maheu-Giroux, M., Lambert, G., Mishra, S., Apelian, H., Messier-Peet, M., Otis, J., Grace, D., Hart, T. A., Moore, D. M., Lachowsky, N. J., \& Cox, J. (2020). Combination HIV prevention strategies among Montreal gay, bisexual, and other men who have sex with men in the PrEP era: A latent class analysis. AIDS and Behavior. https://doi. org/10.1007/s10461-020-02965-4

Drückler, S., van Rooijen, M. S., \& de Vries, H. J. C. (2018). Chemsex among men who have sex with men: A sexualized drug use survey among clients of the sexually transmitted infection outpatient clinic and users of a gay dating app in Amsterdam, the Netherlands. Sexually Transmitted Diseases, 45(5), 325-331. https://doi.org/10.1097/ OLQ.0000000000000753

Dubov, A., Altice, F. L., \& Fraenkel, L. (2018). An information-motivation-behavioral skills model of PrEP uptake. AIDS and Behavior, 22(11), 3603-3616. https://doi.org/10.1007/ s10461-018-2095-4

Dubov, A., Galbo, P., Altice, F. L., \& Fraenkel, L. (2018). Stigma and shame experiences by MSM who take PrEP for HIV prevention: A qualitative study. American Journal of Mens Health, 12(6), 1843-1854. https://doi.org/10.1177/1557988318797437

Dubov, A., Ogunbajo, A., Altice, F. L., \& Fraenkel, L. (2019). Optimizing access to PrEP based on MSM preferences: results of a discrete choice experiment. AIDS Care, 31(5), 545-553. https://doi.org/10.1080/09540121.2018.1557590

Dunville, R., Harper, C., Johns, M. M., Heim Viox, M., Avripas, S., Fordyce, E., Stern, M., Schlissel, A., Carpenter, R., \& Michaels, S. (2020). Awareness and willingness to use biomedical prevention strategies for HIV among sexual and gender minority youth: Results from a national survey. Journal of Adolescent Health, 1-8. https://doi.org/10.1016/j.jadohealth.2020.05.032 
Eaton, L. A., \& Kalichman, S. C. (2007). Risk compensation in HIV prevention: Implications for vaccines, microbicides, and other biomedical HIV prevention technologies. Current HIV/AIDS Reports, 4(4), 165-172. https://doi.org/10.1007/s11904-007-0024-7

Eaton, L. A., Kalichman, S. C., O’Connell, D. A., \& Karchner, W. D. (2009). A strategy for selecting sexual partners believed to pose little/no risks for HIV: serosorting and its implications for HIV transmission. AIDS Care, 21(10), 1279-1288. https://doi. org/10.1080/09540120902803208

Eaton, L. A., Kalichman, S. C., Price, D., Finneran, S., Allen, A., \& Maksut, J. (2017). Stigma and conspiracy beliefs related to pre-exposure prophylaxis (PrEP) and interest in using PrEP among black and white men and transgender women who have sex with men. Aids and Behavior, 21(5), 1236-1246. https://doi.org/10.1007/s10461-017-1690-0

Eaton, L. A., Matthews, D. D., Bukowski, L. A., Friedman, M. R., Chandler, C. J., Whitfield, D. L., Sang, J. M., \& Stall, R. D. (2018). Elevated HIV prevalence and correlates of PrEP use among a community sample of black men who have sex with men. Journal of Acquired Immune Deficiency Syndromes, 79(3), 339-346. https://doi.org/10.1097/ QAI.0000000000001822

Eisinger, R. W., Dieffenbach, C. W., \& Fauci, A. S. (2019). HIV viral load and transmissibility of HIV infection. JAMA: The Journal of the American Medical Association, 321(5), 451. https://doi.org/10.1001/jama.2018.21167

Elsesser, S. A., Oldenburg, C. E., Biello, K. B., Mimiaga, M. J., Safren, S. A., Egan, J. E., Novak, D. S., Krakower, D. S., Stall, R., \& Mayer, K. H. (2016). Seasons of risk: Anticipated behavior on vacation and interest in episodic antiretroviral pre-exposure prophylaxis (PrEP) among a large national sample of U.S. men who have sex with men (MSM). AIDS and Behavior, 20(7), 1400-1407. https://doi.org/10.1007/s10461-015-1238-0

European Centre for Disease Prevention and Control. (2019a). EMIS-2017: The European men-who-have-sex-with-men internet survey: Key findings from 50 countries. https://www. ecdc.europa.eu/en/publications-data/emis-2017-european-men-who-have-sex-men-internet-survey

European Centre for Disease Prevention and Control. (2019b). HIV/AIDS surveillance in Europe 2019 - 2018 data. https://doi.org/doi:10.2900/47834

European Centre for Disease Prevention and Control. (2019c). Pre-exposure prophylaxis for HIV prevention in Europe and Central Asia. Monitoring implementation of the Dublin Declaration on partnership to fight HIV/AIDS in Europe and Central Asia - 2018/19 progress report. https://www.ecdc.europa.eu/sites/default/files/documents/HIV-pre-exposure-prophylaxis-evidence-2019_0.pdf 
European Medicines Agency. (2016). First medicine for HIV pre-exposure prophylaxis recommended for approval in the EU. http://www.ema.europa.eu/docs/en_GB/document_library/Press_release/2016/07/WC500210885.pdf

Evans, A. R., Wiggins, R. D., Mercer, C. H., Bolding, G. J., \& Elford, J. (2007). Men who have sex with men in Great Britain: Comparison of a self-selected internet sample with a national probability sample. Sexually Transmitted Infections, 83(3), 200-205. https://doi. org/10.1136/sti.2006.023283

Ferrer, L., Folch, C., Fernandez-Davila, P., Garcia, A., Morales, A., Belda, J., Susperregui, A. R., \& Casabona, J. (2016). Awareness of pre-exposure prophylaxis for HIV, willingness to use it and potential barriers or facilitators to uptake among men who have sex with men in Spain. AIDS and Behavior, 20(7), 1423-1433. https://doi.org/10.1007/s10461-016-1379-9

Fonner, V. A., Dalglish, S. L., Kennedy, C. E., Baggaley, R., O’Reilly, K. R., Koechlin, F. M., Rodolph, M., Hodges-Mameletzis, I., \& Grant, R. M. (2016). Effectiveness and safety of oral HIV preexposure prophylaxis for all populations. AIDS, 30(12), 1973-1983. https:// doi.org/10.1097/QAD.0000000000001145

Frankis, J., Young, I., Flowers, P., \& McDaid, L. (2016). Who will use pre-exposure prophylaxis (PrEP) and why?: Understanding PrEP awareness and acceptability amongst men who have sex with men in the UK - A mixed methods study. PLoS ONE, 11(4), e0151385. https://doi.org/10.1371/journal.pone.0151385

Franks, J., Hirsch-Moverman, Y., Loquere, A. S., Amico, K. R., Grant, R. M., Dye, B. J., Rivera, Y., Gamboa, R., \& Mannheimer, S. B. (2018). Sex, PrEP, and stigma: Experiences with HIV pre-exposure prophylaxis among New York City MSM participating in the HPTN 067/ADAPT study. AIDS and Behavior, 22(4), 1139-1149. https://doi.org/10.1007/ s10461-017-1964-6

Freeborn, K., \& Portillo, C. J. (2018). Does pre-exposure prophylaxis for HIV prevention in men who have sex with men change risk behaviour? A systematic review. Journal of Clinical Nursing, 27(17-18), 3254-3265. https://doi.org/10.1111/jocn.13990

Gafos, M., Horne, R., Nutland, W., Bell, G., Rae, C., Wayal, S., Rayment, M., Clarke, A., Schembri, G., Gilson, R., McOwan, A., Sullivan, A., Fox, J., Apea, V., Dewsnap, C., Dolling, D., White, E., Brodnicki, E., Wood, G., ... McCormack, S. (2019). The context of sexual risk behaviour among men who have sex with men seeking PrEP, and the impact of PrEP on sexual behaviour. AIDS and Behavior, 23(7), 1708-1720. https://doi.org/10.1007/s10461018-2300-5

Galea, J., Cook, R., Pickett, J., \& Gorbach, P. (2016). "PrEP in the wild” - Preliminary results from a global survey on "informal" PrEP use in settings where it is approved and where it 
is not.

GGD Amsterdam. (2017). Over InPrEP. https://www.ggd.amsterdam.nl/infectieziekten/soahiv-sense/prep/inprep/

Gibbons, F. X., Gerrard, M., Ouellette, J. A., \& Burzette, R. (1998). Cognitive antecedents to adolescent health risk: Discriminating between behavioral intention and behavioral willingness. Psychology and Health, 13(2), 319-339. https://doi.org/10.1080/08870449808406754

Gilead. (2012). First agent indicated for Uninfected adults at high risk of acquiring HIV through sex. 3-5. http://www.gilead.com/news/press-releases/2012/7/us-food-and-drug-administration-approves-gileads-truvada-for-reducing-the-risk-of-acquiring-hiv

Golden, M. R., Stekler, J., Hughes, J. P., \& Wood, R. W. (2008). HIV serosorting in men who have sex with men: Is it safe? JAIDS Journal of Acquired Immune Deficiency Syndromes, 49(2), 212-218. https://doi.org/10.1097/QAI.0b013e31818455e8

Golub, S. A. (2018). PrEP stigma: Implicit and explicit drivers of disparity. Current HIV/AIDS Reports, 15(2), 190-197. https://doi.org/10.1007/s11904-018-0385-0

Golub, S. A., Fikslin, R. A., Goldberg, M. H., Peña, S. M., \& Radix, A. (2019). Predictors of PrEP uptake among patients with equivalent access. AIDS and Behavior, 23(7), 19171924. https://doi.org/10.1007/s10461-018-2376-y

Golub, S. A., Fikslin, R. A., Starbuck, L., \& Klein, A. (2019). High rates of PrEP eligibility but low rates of PrEP access among a national sample of transmasculine individuals. JAIDS Journal of Acquired Immune Deficiency Syndromes, 82(1), E1-E7. https://doi.org/10.1097/ QAI.0000000000002116

Golub, S. A., Gamarel, K. E., Rendina, H. J., Surace, A., \& Lelutiu-Weinberger, C. L. (2013). From efficacy to effectiveness: Facilitators and barriers to PrEP acceptability and motivations for adherence among MSM and transgender women in New York City. AIDS Patient Care and STDs, 27(4), 248-254. https://doi.org/10.1089/apc.2012.0419

Golub, S. A., Kowalczyk, W., Weinberger, C. L., \& Parsons, J. T. (2010). Pre-exposure prophylaxis and predicted condom use among high-risk men who have sex with men. Journal of Acquired Immune Deficiency Syndromes, 54(5), 548-555. https://doi.org/10.1097/QAI. 0b013e3181e19a54

Golub, S. A., Lelutiu-Weinberger, C., \& Surace, A. (2018). Experimental investigation of implicit HIV and preexposure prophylaxis stigma. JAIDS Journal of Acquired Immune Deficiency Syndromes, 77(3), 264-271. https://doi.org/10.1097/QAI.0000000000001592

Goparaju, L., Praschan, N. C., Jeanpiere, L. W., Experton, L. S., Young, M. A., \& Kassaye, S. (2017). Stigma, partners, providers and costs: Potential barriers to PrEP uptake among US women. Journal of AIDS \& Clinical Research, 08(09). https://doi.org/10.4172/2155- 


\subsection{0}

Grace, D., Jollimore, J., MacPherson, P., Strang, M. J. P., \& Tan, D. H. S. (2018). The pre-exposure prophylaxis-stigma paradox: Learning from Canada's first wave of PrEP users. AIDS Patient Care and STDs, 32(1), 24-30. https://doi.org/10.1089/apc.2017.0153

Grant, R. M., Anderson, P. L., McMahan, V., Liu, A., Amico, K. R., Mehrotra, M., Hosek, S., Mosquera, C., Casapia, M., Montoya, O., Buchbinder, S., Veloso, V. G., Mayer, K., Chariyalertsak, S., Bekker, L.-G., Kallas, E. G., Schechter, M., Guanira, J., Bushman, L., ... Glidden, D. V. (2014). Uptake of pre-exposure prophylaxis, sexual practices, and HIV incidence in men and transgender women who have sex with men: A cohort study. The Lancet Infectious Diseases, 14(9), 820-829. https://doi.org/10.1016/S1473-3099(14)70847-3

Grant, R. M., \& Koester, K. A. (2016). What people want from sex and PrEP. Current Opinion in HIV and AIDS, 11(1), 3-9. https://doi.org/10.1097/COH.0000000000000216

Grant, R. M., Lama, J. R., Anderson, P. L., McMahan, V., Liu, A. Y., Vargas, L., Goicochea, P., Casapía, M., Guanira-Carranza, J. V., Ramirez-Cardich, M. E., Montoya-Herrera, O., Fernández, T., Veloso, V. G., Buchbinder, S. P., Chariyalertsak, S., Schechter, M., Bekker, L.-G., Mayer, K. H., Kallás, E. G., ... Glidden, D. V. (2010). Preexposure chemoprophylaxis for HIV prevention in men who have sex with men. New England Journal of Medicine, 363(27), 2587-2599. https://doi.org/10.1056/NEJMoa1011205

Grov, C., Jonathan Rendina, H., Patel, V. V., Kelvin, E., Anastos, K., \& Parsons, J. T. (2018). Prevalence of and factors associated with the use of HIV serosorting and other biomedical prevention strategies among men who have sex with men in a US nationwide survey. AIDS and Behavior, 22(8), 2743-2755. https://doi.org/10.1007/s10461-018-2084-7

Grov, C., Rendina, H. J., Whitfield, T. H. F., Ventuneac, A., \& Parsons, J. T. (2016). Changes in familiarity with and willingness to take preexposure prophylaxis in a longitudinal study of highly sexually active gay and bisexual men. LGBT Health, 3(4), 252-257. https://doi. org/10.1089/lgbt.2015.0123

Grulich, A. E., Guy, R., Amin, J., Jin, F., Selvey, C., Holden, J., Schmidt, H. M. A., Zablotska, I., Price, K., Whittaker, B., Chant, K., Cooper, C., McGill, S., Telfer, B., Yeung, B., Levitt, G., Ogilvie, E. E., Dharan, N. J., Hammoud, M. A., ... Parkhill, N. (2018). Population-level effectiveness of rapid, targeted, high-coverage roll-out of HIV pre-exposure prophylaxis in men who have sex with men: the EPIC-NSW prospective cohort study. The Lancet HIV, 5(11), e629-e637. https://doi.org/10.1016/S2352-3018(18)30215-7

Haas, S. M., Perazzo, J. D., Ruffino, A. H., Ancona, R. M., \& Lyons, M. (2017). The know now project: Facilitated serosorting in HIV-status sexual partner communication. AIDS Education and Prevention, 29(5), 432-442. https://doi.org/10.1521/aeap.2017.29.5.432 
Haire, B. (2015). Preexposure prophylaxis-related stigma: strategies to improve uptake and adherence \&amp;ndash; a narrative review. HIV/AIDS - Research and Palliative Care, 7 , 241. https://doi.org/10.2147/HIV.S72419

Hammack, P. L., Meyer, I. H., Krueger, E. A., Lightfoot, M., \& Frost, D. M. (2018). HIV testing and pre-exposure prophylaxis (PrEP) use, familiarity, and attitudes among gay and bisexual men in the United States: A national probability sample of three birth cohorts. PLoS ONE, 13(9). https://doi.org/10.1371/journal.pone.0202806

Hannaford, A., Lipshie-Williams, M., Starrels, J. L., Arnsten, J. H., Rizzuto, J., Cohen, P., Jacobs, D., \& Patel, V. V. (2018). The use of online posts to identify barriers to and facilitators of HIV pre-exposure prophylaxis (PrEP) among men who have sex with men: A comparison to a systematic review of the peer-reviewed literature. AIDS and Behavior, 22(4), 1080-1095. https://doi.org/10.1007/s10461-017-2011-3

Hayes, A. F. (2017). Introduction to mediation, moderation, and conditional process analysis: A regression-based approach. Guilford Publications.

Hayes, A. F., \& Matthes, J. (2009). Computational procedures for probing interactions in OLS and logistic regression: SPSS and SAS implementations. Behavior Research Methods, 41(3), 924-936. https://doi.org/10.3758/BRM.41.3.924

Hayes, R., Schmidt, A. J., Pharris, A., Azad, Y., Brown, A. E., Weatherburn, P., \& Hickson, F. (2019). Estimating the 'PrEP Gap': how implementation and access to PrEP differ between countries in Europe and Central Asia in 2019. Eurosurveillance, 24(41), 1-7. https://doi. org/10.2807/1560-7917.ES.2019.24.41.1900598

Hess, K. L., Hu, X., Lansky, A., Mermin, J., \& Hall, H. I. (2017). Lifetime risk of a diagnosis of HIV infection in the United States. Annals of Epidemiology, 27(4), 238-243. https://doi. org/10.1016/j.annepidem.2017.02.003

Hodges-Mameletzis, I., Dalal, S., Msimanga-Radebe, B., Rodolph, M., \& Baggaley, R. (2018). Going global: The adoption of the World Health Organization's enabling recommendation on oral pre-exposure prophylaxis for HIV. Sexual Health, 15(6), 489-500. https://doi. org/10.1071/SH18125

Hojilla, J. C., Koester, K. A., Cohen, S. E., Buchbinder, S., Ladzekpo, D., Matheson, T., \& Liu, A. Y. (2016). Sexual behavior, risk compensation, and HIV prevention strategies among participants in the San Francisco PrEP demonstration project: A qualitative analysis of counseling notes. AIDS and Behavior, 20(7), 1461-1469. https://doi.org/10.1007/s10461015-1055-5

Holloway, I. W., Dougherty, R., Gildner, J., Beougher, S. C., Pulsipher, C., Montoya, J. A., Plant, A., \& Leibowitz, A. (2017). Brief report: PrEP uptake, adherence, and discon- 
tinuation among California YMSM using geosocial networking applications. Journal of Acquired Immune Deficiency Syndromes, 74(1), 15-20. https://doi.org/10.1097/ QAI.0000000000001164

Holt, M., Draper, B. L., Pedrana, A. E., Wilkinson, A. L., \& Stoové, M. (2018). Comfort relying on HIV pre-exposure prophylaxis and treatment as prevention for condomless sex: Results of an online survey of Australian gay and bisexual men. AIDS and Behavior, 22(11), 1-10. https://doi.org/10.1007/s10461-018-2097-2

Holt, M., Lea, T., Bear, B., Halliday, D., Ellard, J., Murphy, D., Kolstee, J., \& de Wit, J. (2019). Trends in attitudes to and the use of HIV pre-exposure prophylaxis by Australian gay and bisexual men, 2011-2017: Implications for further implementation from a diffusion of innovations perspective. AIDS and Behavior, 23(7), 1939-1950. https://doi.org/10.1007/ s10461-018-2368-y

Holt, M., Lea, T., Mao, L., Kolstee, J., Zablotska, I., Duck, T., Allan, B., West, M., Lee, E., Hull, P., Grulich, A., De Wit, J., \& Prestage, G. (2018). Community-level changes in condom use and uptake of HIV pre-exposure prophylaxis by gay and bisexual men in Melbourne and Sydney, Australia: results of repeated behavioural surveillance in 2013-17. The Lancet HIV. https://doi.org/10.1016/S2352-3018(18)30072-9

Holt, M., Murphy, D. A., Callander, D., Ellard, J., Rosengarten, M., Kippax, S. C., \& de Wit, J. B. F. (2012). Willingness to use HIV pre-exposure prophylaxis and the likelihood of decreased condom use are both associated with unprotected anal intercourse and the perceived likelihood of becoming HIV positive among Australian gay and bisexual men. Sexually Transmitted Infections, 88(4), 258-263. https://doi.org/10.1136/sextrans-2011-050312

Hoornenborg, E., Achterbergh, R. C. A., Schim van der Loeff, M. F., Davidovich, U., Hogewoning, A., de Vries, H. J. C., Schinkel, J., Prins, M., van de Laar, T. J. W., \& Amsterdam PrEP Project team in the HIV Transmission Elimination AMsterdam Initiative, M. study group. (2017). MSM starting preexposure prophylaxis are at risk of hepatitis $C$ virus infection. AIDS, 31(11), 1603-1610. https://doi.org/10.1097/QAD.0000000000001522

Hoornenborg, E., Coyer, L., Achterbergh, R. C. A., Matser, A., Schim van der Loeff, M. F., Boyd, A., van Duijnhoven, Y. T. H. P., Bruisten, S., Oostvogel, P., Davidovich, U., Hogewoning, A., Prins, M., \& de Vries, H. J. C. (2019). Sexual behaviour and incidence of HIV and sexually transmitted infections among men who have sex with men using daily and event-driven pre-exposure prophylaxis in AMPrEP: 2 year results from a demonstration study. The Lancet HIV, 6(7), e447-e455. https://doi.org/10.1016/S2352-3018(19)30136-5 Hoornenborg, E., Coyer, L., van Laarhoven, A., Achterbergh, R., de Vries, H., Prins, 
M., \& van der Loeff, M. S. (2018). Change in sexual risk behaviour after 6 months of pre-exposure prophylaxis use. AIDS, 32(11), 1527-1532. https://doi.org/10.1097/ QAD.0000000000001874

Hoornenborg, E., Krakower, D. S., Prins, M., \& Mayer, K. H. (2017). Pre-exposure prophylaxis for MSM and transgender persons in early adopting countries. AIDS, 31(16), 2179-2191. https://doi.org/10.1097/QAD.0000000000001627

Hoornenborg, E., \& Rijnders, B. (2016). HIV Pre-expositie profylaxe (PrEP) richtlijn Nederland. http://richtlijnhiv.nvhb.nl/images/2/22/PrEP-richtlijn-Nederland-8-september2016-met-logos.pdf

Hoornenborg, E., \& Rijnders, B. (2019). HIV Pre-expositie profylaxe (PrEP) richtlijn Nederland (Issue gereviseerde versie 2). https://sekshag.nhg.org/sites/default/files/content/ nhg_org/images/prep_richtlijn_nederland_versie_2_dd_15_april_2019.pdf

Jaspal, R., \& Daramilas, C. (2016). Perceptions of pre-exposure prophylaxis (PrEP) among HIV-negative and HIV-positive men who have sex with men (MSM). Cogent Medicine, 3(1). https://doi.org/10.1080/2331205x.2016.1256850

Jonas, K. J., \& Yaemim, N. (2018). HIV prevention after discontinuing pre-exposure prophylaxis: Conclusions from a case study. Frontiers in Public Health, 6(May), 1-4. https://doi. org/10.3389/fpubh.2018.00137

Jones, J., Sullivan, P. S., \& Curran, J. W. (2019). Progress in the HIV epidemic: Identifying goals and measuring success. PLOS Medicine, 16(1), e1002729. https://doi.org/10.1371/ journal.pmed.1002729

Juusola, J. L., Brandeau, M. L., Owens, D. K., \& Bendavid, E. (2012). The cost-effectiveness of preexposure prophylaxis for HIV prevention in the United States in men who have sex with men. Annals of Internal Medicine, 156(8), 541-550.

Kamitani, E., Johnson, W. D., Wichser, M. E., Adegbite, Adebukola, H., Mullins, M. M., \& Sipe, T. A. (2020). Growth in proportion and disparities of HIV PrEP use among key populations identified in the United States national goals. JAIDS Journal of Acquired Immune Deficiency Syndromes, 4, 379-386. https://doi.org/10.1097/QAI.0000000000002345

Kamitani, E., Wichser, M. E., Adegbite, A. H., Mullins, M. M., Johnson, W. D., Crouch, P. C., \& Sipe, T. A. (2018). Increasing prevalence of self-reported HIV preexposure prophylaxis use in published surveys: A systematic review and meta-analysis. AIDS, 32(17), 2633-2635. https://doi.org/10.1097/QAD.0000000000001983

Karris, M. Y., Beekmann, S. E., Mehta, S. R., Anderson, C. M., \& Polgreen, P. M. (2014). Are we prepped for preexposure prophylaxis (PrEP)? Provider opinions on the real-world use of PrEP in the United States and Canada. Clinical Infectious Diseases, 58(5), 704-712. 


\section{https://doi.org/10.1093/cid/cit796}

Kay, E. S., \& Pinto, R. M. (2020). Is insurance a barrier to HIV preexposure prophylaxis? Clarifying the issue. American Journal of Public Health, 110(1), 61-64. https://doi.org/10.2105/ AJPH.2019.305389

Keen, P., Hammoud, M. A., Bourne, A., Bavinton, B. R., Holt, M., Vaccher, S., Haire, B., Saxton, P., Jin, F., Maher, L., Grulich, A. E., \& Prestage, G. (2020). Use of HIV pre-exposure prophylaxis (PrEP) associated with lower HIV anxiety among gay and bisexual men in Australia who are at high risk of HIV infection. JAIDS Journal of Acquired Immune Deficiency Syndromes, 83(2), 119-125. https://doi.org/10.1097/QAI.0000000000002232

Keene, L. C., Dehlin, J. M., Pickett, J., Berringer, K. R., Little, I., Tsang, A., Bouris, A. M., \& Schneider, J. A. (2020). \#PrEP4Love: success and stigma following release of the first sex-positive PrEP public health campaign. Culture, Health \& Sexuality, 0(0), 1-17. https://doi.org/10.1080/13691058.2020.1715482

Kelley, C. F., Kahle, E., Siegler, A., Sanchez, T., del Rio, C., Sullivan, P. S., \& Rosenberg, E. S. (2015). Applying a PrEP continuum of care for men who have sex with men in Atlanta, Georgia. Clinical Infectious Diseases, 61(10), 1590-1597. https://doi.org/10.1093/cid/ civ664

Kessler, J., Myers, J. E., Nucifora, K. A., Mensah, N., Toohey, C., Khademi, A., Cutler, B., \& Braithwaite, S. (2014). Evaluating the impact of prioritization of antiretroviral pre-exposure prophylaxis in New York. Aids, 28(18), 2683-2691.

King, H. L., Keller, S. B., Giancola, M. A., Rodriguez, D. A., Chau, J. J., Young, J. A., Little, S. J., \& Smith, D. M. (2014). Pre-Exposure Prophylaxis Accessibility Research and Evaluation (PrEPARE Study). AIDS and Behavior, 18(9), 1722-1725. https://doi.org/10.1007/ s10461-014-0845-5

Kirby, T., \& Thornber-Dunwell, M. (2014). Uptake of PrEP for HIV slow among MSM. The Lancet, 383(9915), 399-400. https://doi.org/10.1016/S0140-6736(14)60137-9

Koester, K. A., Amico, R. K., Gilmore, H., Liu, A., McMahan, V., Mayer, K., Hosek, S., \& Grant, R. (2017). Risk, safety and sex among male PrEP users: time for a new understanding. Culture, Health and Sexuality, 19(12). https://doi.org/10.1080/13691058.2017 .1310927

Koester, K. A., Erguera, X. A., Kang Dufour, M.-S., Udoh, I., Burack, J. H., Grant, R. M., \& Myers, J. J. (2018). “Losing the phobia:” Understanding how HIV pre-exposure prophylaxis facilitates bridging the serodivide among men who have sex with men. Frontiers in Public Health, 6(September), 1-12. https://doi.org/10.3389/fpubh.2018.00250

Koppe, U., Marcus, U., Albrecht, S., Jansen, K., Jessen, H., Gunsenheimer-Bartmeyer, B., \& 
Bremer, V. (2019). Factors associated with the informal use of HIV pre-exposure prophylaxis in Germany: a cross-sectional study. Journal of the International AIDS Society, 22(10), e25395. https://doi.org/10.1002/jia2.25395

Krakower, D. S., Daskalakis, D. C., Feinberg, J., \& Marcus, J. L. (2020). Tenofovir Alafenamide for HIV preexposure prophylaxis: What can we DISCOVER about its true value? Annals of Internal Medicine, 172(4), 281. https://doi.org/10.7326/M19-3337

Kubicek, K., Arauz-Cuadra, C., \& Kipke, M. D. (2015). Attitudes and perceptions of biomedical HIV prevention methods: Voices from young men who have sex with men. Archives of Sexual Behavior, 44(2), 487-497. https://doi.org/10.1007/s10508-014-0398-8

Kuhns, L. M., Hotton, A. L., Schneider, J., Garofalo, R., \& Fujimoto, K. (2017). Use of pre-exposure prophylaxis (PrEP) in young men who have sex with men is associated with race, sexual risk behavior and peer network size. AIDS and Behavior, 21(5), 1376-1382. https:// doi.org/10.1007/s10461-017-1739-0

Kuo, C., Giovenco, D., DeAtley, T., Hoare, J., Underhill, K., Atujuna, M., Mathews, C., Stein, D. J., Brown, L. K., \& Operario, D. (2020). Recreational use of HIV antiretroviral medication and implications for HIV pre-exposure prophylaxis and treatment. AIDS and Behavior, 0123456789. https://doi.org/10.1007/s10461-020-02821-5

Kurtz, S. P., \& Buttram, M. E. (2016). Misunderstanding of pre-exposure prophylaxis use among men who have sex with men: Public health and policy implications. LGBT Health, 3(6), 461-464. https://doi.org/10.1089/lgbt.2015.0069

Kuyper, L., Fernee, H., \& Keuzenkamp, S. (2016). A comparative analysis of a community and general sample of lesbian, gay, and bisexual individuals. Archives of Sexual Behavior, 45(3), 683-693. https://doi.org/10.1007/s10508-014-0457-1

Lal, L., Audsley, J., Murphy, D. A., Fairley, C. K., Stoove, M., Roth, N., Moore, R., Tee, B. K., Puratmaja, N., Anderson, P. L., Leslie, D., Grant, R. M., De Wit, J., Wright, E., ElHayek, C., Petoumenos, K., Grulich, A., Zablotska, I., Crowe, S., ... Willcox, J. (2017). Medication adherence, condom use and sexually transmitted infections in Australian preexposure prophylaxis users. AIDS, 31(12), 1709-1714. https://doi.org/10.1097/ QAD.0000000000001519

Leusink, P. (2020). De seksuele moraal van de huisarts: l'histoire se répète. Huisarts En Wetenschap, 1-4. https://doi.org/10.1007/s12445-020-0546-4

Lewnard, J. A., \& Berrang-Ford, L. (2014). Internet-based partner selection and risk for unprotected anal intercourse in sexual encounters among men who have sex with men: A meta-analysis of observational studies. Sexually Transmitted Infections, 90(4), 290-296. https://doi.org/10.1136/sextrans-2013-051332 
Liu, A. Y., Vittinghoff, E., Chillag, K., Mayer, K., Thompson, M., Grohskopf, L., Colfax, G., Pathak, S., Gvetadze, R., O’Hara, B., Collins, B., Ackers, M., Paxton, L., \& Buchbinder, S. P. (2013). Sexual risk behavior among HIV-uninfected men who have sex with men (MSM) participating in a tenofovir pre-exposure prophylaxis (PrEP) randomized trial in the United States. Journal of Acquired Immune Deficiency Syndromes, 64(1), 87-94. https://doi.org/10.1097/QAI.0b013e31828f097a

Mabire, X., Puppo, C., Morel, S., Mora, M., Rojas Castro, D., Chas, J., Cua, E., Pintado, C., Suzan-Monti, M., Spire, B., Molina, J. M., \& Préau, M. (2019). Pleasure and PrEP: Pleasure-seeking plays a role in prevention choices and could lead to PrEP initiation. American Journal of Men's Health, 13. https://doi.org/10.1177/1557988319827396

Macapagal, K., Kraus, A., Korpak, A. K., Jozsa, K., \& Moskowitz, D. A. (2020). PrEP awareness, uptake, barriers, and correlates among adolescents assigned male at birth who have sex with males in the U.S. Archives of Sexual Behavior, 49(1), 113-124. https://doi. org/10.1007/s10508-019-1429-2

Malone, J., Syvertsen, J. L., Johnson, B. E., Mimiaga, M. J., Mayer, K. H., \& Bazzi, A. R. (2018). Negotiating sexual safety in the era of biomedical HIV prevention: relationship dynamics among male couples using pre-exposure prophylaxis. Culture, Health and Sexuality, 20(6), 658-672. https://doi.org/10.1080/13691058.2017.1368711

Marcus, J. L., Hurley, L. B., Hare, C. B., Silverberg, M. J., \& Volk, J. E. (2016). Disparities in uptake of HIV preexposure prophylaxis in a large integrated health care system. American Journal of Public Health, 106(10), e2-e3. https://doi.org/10.2105/AJPH.2016.303339

Martinez, J. E., \& Jonas, K. J. (2019). Pre-exposure prophylaxis sorting among men who have sex with men. AIDS Care, 31(3), 388-396. https://doi.org/10.1080/09540121.2018.15332 29

Martinez, J. E., \& Jonas, K. J. (2020). Social judgments of sexual behavior and use of HIV pre-exposure prophylaxis. Social Cognition, 38(1), 1-20. https://doi.org/10.1521/ soco.2020.38.1.01

Maxwell, S., Shahmanesh, M., \& Gafos, M. (2019). Chemsex behaviours among men who have sex with men: A systematic review of the literature. International Journal of Drug Policy, 63, 74-89. https://doi.org/10.1016/j.drugpo.2018.11.014

Mayor, S. (2016). NHS to fund large trial of pre-exposure prophylaxis for HIV prevention. BMJ, 355(December), i6537. https://doi.org/10.1136/bmj.i6537

McCall, H., Adams, N., Mason, D., \& Willis, J. (2015). What is chemsex and why does it matter? BMJ, h5790. https://doi.org/doi:10.1136/bmj.h5790

McCormack, S., Dunn, D. T., Desai, M., Dolling, D. I., Gafos, M., Gilson, R., Sullivan, A. K., 
Clarke, A., Reeves, I., Schembri, G., Mackie, N., Bowman, C., Lacey, C. J., Apea, V., Brady, M., Fox, J., Taylor, S., Antonucci, S., Khoo, S. H., ... Gill, O. N. (2016). Pre-exposure prophylaxis to prevent the acquisition of HIV-1 infection (PROUD): Effectiveness results from the pilot phase of a pragmatic open-label randomised trial. The Lancet, 387(10013), 53-60. https://doi.org/10.1016/S0140-6736(15)00056-2

Medina, M. M., Crowley, C., Montgomery, M. C., Tributino, A., Almonte, A., Sowemimo-Coker, G., Nunn, A., \& Chan, P. A. (2019). Disclosure of HIV serostatus and pre-exposure prophylaxis use on internet hookup sites among men who have sex with men. AIDS and Behavior, 23(7), 1681-1688. https://doi.org/10.1007/s10461-018-2286-Z

Medland, N., Guy, R., Grulich, A., Bavinton, B., Keen, P., Ellard, J., Jin, F., Paynter, H., \& McManus, H. (2020). Successful national PrEP scale-up in Australia: Evaluation of uptake, adherence, discontinuation and HIV seroconversion from April 2018 to September 2019 using national dispensing data. 23rd International AIDS Conference, abstract OAC0807.

Meunier, É., \& Siegel, K. (2019). Sex club/party attendance and STI among men who have sex with men: Results from an online survey in New York City. Sexually Transmitted Infections, 95(8), 584-587. https://doi.org/10.1136/sextrans-2018-053816

Meyer, I. H., \& Wilson, P. A. (2009). Sampling lesbian, gay, and bisexual populations. Journal of Counseling Psychology, 56(1), 23-31. https://doi.org/10.1037/a0014587

Milam, J., Jain, S., Dubé, M. P., Daar, E. S., Sun, X., Corado, K., Ellorin, E., Blumenthal, J., Haubrich, R., Moore, D. J., \& Morris, S. R. (2019). Sexual risk compensation in a pre-exposure prophylaxis demonstration study among individuals at risk of HIV. JAIDS Journal of Acquired Immune Deficiency Syndromes, 80(1), e9-e13. https://doi.org/10.1097/ QAI.0000000000001885

Ministry of Health Welfare and Sport. (2018). Letter on the preventive use of antiretroviral drugs (PrEP). https://www.government.nl/documents/parliamentary-documents/2018/07/10/ letter-on-preventive-use-of-antiretroviral-drugs-prep

Ministry of Health Welfare and Sport. (2019). Kamerbrief over start van verstrekking en medische begeleiding van PrEP. https:/www.rijksoverheid.nl/documenten/kamerstukken/2019/06/26/kamerbrief-over-start-van-verstrekking-en-medische-begeleiding-van-prep

Moeller, R. W., Seehuus, M., Wahl, L., \& Gratch, I. (2020). Use of PrEP, sexual behaviors and mental health correlates in a sample of gay, bisexual and other men who have sex with men. Journal of Gay and Lesbian Mental Health, 24(1), 94-111. https://doi.org/10.1080/1 9359705.2019.1688216

Molina, J.-M., Capitant, C., Spire, B., Pialoux, G., Cotte, L., Charreau, I., Tremblay, C., Le Gall, 
J.-M., Cua, E., Pasquet, A., Raff, F., Pintado, C., Chidiac, C., Chas, J., Charbonneau, P., Delaugerre, C., Suzan-Monti, M., Loze, B., Fonsart, J., ... Delfraissy, J.-F. (2015). On-demand preexposure prophylaxis in men at high risk for HIV-1 infection. New England Journal of Medicine, 373(23), 2237-2246. https://doi.org/10.1056/NEJMoa1506273

Molina, J. M., Charreau, I., Spire, B., Cotte, L., Chas, J., Capitant, C., Tremblay, C., Rojas-Castro, D., Cua, E., Pasquet, A., Bernaud, C., Pintado, C., Delaugerre, C., Sagaon-Teyssier, L., Mestre, S. Le, Chidiac, C., Pialoux, G., Ponscarme, D., Fonsart, J., ... Rabian, C. (2017). Efficacy, safety, and effect on sexual behaviour of on-demand pre-exposure prophylaxis for HIV in men who have sex with men: an observational cohort study. The Lancet HIV, 4(9). https://doi.org/10.1016/S2352-3018(17)30089-9

Morgan, E., Moran, K., Ryan, D. T., Mustanski, B., \& Newcomb, M. E. (2018). Threefold increase in PrEP uptake over time with high adherence among young men who have sex with men in Chicago. AIDS and Behavior, 22(11), 1-8. https://doi.org/10.1007/s10461018-2122-5

Moskowitz, D. A., Macapagal, K., Mongrella, M., Pérez-Cardona, L., Newcomb, M. E., \& Mustanski, B. (2020). What if my dad finds out!?: Assessing adolescent men who have sex with men's perceptions about parents as barriers to PrEP uptake. AIDS and Behavior. https://doi.org/10.1007/s10461-020-02827-z

Murphy, D. A., de Wit, J. B. F., Donohoe, S., \& Adam, P. C. G. (2015). The need to know: HIV status disclosure expectations and practices among non-HIV-positive gay and bisexual men in Australia. AIDS Care, 27(sup1), 90-98. https://doi.org/10.1080/09540121.2015. 1062077

Myers, J. E., Edelstein, Z. R., Daskalakis, D. C., Gandhi, A. D., Misra, K., Rivera, A. V., Salcuni, P. M., Scanlin, K., Udeagu, C. C., \& Braunstein, S. L. (2018). Preexposure prophylaxis monitoring in New York City: A public health approach. American Journal of Public Health, 108, S251-S257. https://doi.org/10.2105/AJPH.2018.304729

Mysior, P., Tenberken, E., Stecher, M., Vehreschild, J.-J., Schafberger, A., Perea, I., Brillen, A.L., Bartmeyer, B., Esser, S., Bremer, V., Marcus, U., Hoffmann, D., \& Streeck, H. (2020). Rapid uptake of pre-exposure prophylaxis after significant price reduction in Germany highlights importance of pre-exposure prophylaxis accessibility. AIDS Research and Human Retroviruses, 36(9), 721-733. https://doi.org/10.1089/aid.2019.0237

Nagington, M., \& Sandset, T. (2020). Putting the NHS England on trial: uncertainty-as-power, evidence and the controversy of PrEP in England. Medical Humanities, 0(0), 1-4. https:// doi.org/10.1136/medhum-2019-011780

NAM Aidsmap. (2017). Belgium, Portugal and Brazil will provide PrEP through their health 
services; Morocco announces a PrEP study. http://www.aidsmap.com/news/jun-2017/belgium-portugal-and-brazil-will-provide-prep-through-their-health-services-morocco

Newcomb, M. E., Mongrella, M. C., Weis, B., McMillen, S. J., \& Mustanski, B. (2016). Partner disclosure of PrEP use and undetectable viral load on geosocial networking apps. JAIDS Journal of Acquired Immune Deficiency Syndromes, 71(2), 200-206. https://doi. org/10.1097/QAI.0000000000000819

Nichols, B. E., Boucher, C. A. B., van der Valk, M., Rijnders, B. J. A., \& van de Vijver, D. A. M. C. (2016). Cost-effectiveness analysis of pre-exposure prophylaxis for HIV-1 prevention in the Netherlands: a mathematical modelling study. The Lancet Infectious Diseases, 16(12), 1423-1429. https://doi.org/10.1016/S1473-3099(16)30311-5

Nöstlinger, C., Reyniers, T., Smekens, T., Apers, H., Laga, M., Wouters, K., \& Vuylsteke, B. (2020). Drug use, depression and sexual risk behaviour: a syndemic among early pre-exposure prophylaxis (PrEP) adopters in Belgium? AIDS Care - Psychological and Socio-Medical Aspects of AIDS/HIV, 32(sup2), 57-64. https://doi.org/10.1080/09540121.2 020.1739218

NPS MedicineWise. (2018). PrEP on the PBS: An opportunity in HIV prevention. https://www. nps.org.au/news/pr-ep-on-the-pbs-an-opportunity-in-hiv-prevention

Okafor, C. N., Gorbach, P. M., Ragsdale, A., Quinn, B., \& Shoptaw, S. (2017). Correlates of preexposure prophylaxis (PrEP) use among men who have sex with men (MSM) in Los Angeles, California. Journal of Urban Health, 94(5), 710-715. https://doi.org/10.1007/ s11524-017-0172-z

Oldenburg, C. E., Nunn, A. S., Montgomery, M., Almonte, A., Mena, L., Patel, R. R., Mayer, K. H., \& Chan, P. A. (2018). Behavioral changes following uptake of HIV pre-exposure prophylaxis among men who have sex with men in a clinical setting. AIDS and Behavior, 22(4), 1075-1079. https://doi.org/10.1007/s10461-017-1701-1

Opdenakker, R. (2006). Advantages and disadvantages of four interview techniques in qualitative research. Forum Qualitative Sozialforschung / Forum: Qualitative Social Research, 7(4), 13. https://doi.org/10.1016/j.jpedsurg.2016.07.009

Orne, J., \& Gall, J. (2019). Converting, monitoring, and policing PrEP citizenship: Biosexual citizenship and the PrEP surveillance regime. Surveillance \& Society, 17(5), 641-661. https://doi.org/10.24908/ss.v17i5.12945

Owens, C., Hubach, R. D., Williams, D., Voorheis, E., Lester, J., Reece, M., \& Dodge, B. (2020). Facilitators and barriers of pre-exposure prophylaxis (PrEP) uptake among rural men who have sex with men living in the Midwestern U.S. Archives of Sexual Behavior, 0123456789. https://doi.org/10.1007/s10508-020-01654-6 
Owens, D. K., Davidson, K. W., Krist, A. H., Barry, M. J., Cabana, M., Caughey, A. B., Curry, S. J., Doubeni, C. A., Epling, J. W., Kubik, M., Landefeld, C. S., Mangione, C. M., Pbert, L., Silverstein, M., Simon, M. A., Tseng, C. W., \& Wong, J. B. (2019). Preexposure prophylaxis for the prevention of HIV infection: US preventive services task force recommendation statement. JAMA - Journal of the American Medical Association, 321(22), 2203-2213. https://doi.org/10.1001/jama.2019.6390

Paparini, S., Nutland, W., Rhodes, T., Nguyen, V.-K., \& Anderson, J. (2018). DIY HIV prevention: What are the experiences of London-based men who have sex with men who source PrEP outside clinical trials? PLOS ONE, 13(8). https://doi.org/10.1371/journal. pone. 0202830

Parikh, U. M., \& Mellors, J. W. (2016). Should we fear resistance from tenofovir/emtricitabine preexposure prophylaxis? Current Opinion in HIV and AIDS, 11(1), 49-55. https://doi. org/10.1097/COH.0000000000000209

Parsons, J. T., Rendina, H. J., Lassiter, J. M., Whitfield, T. H. F., Starks, T. J., \& Grov, C. (2017). Uptake of HIV pre-exposure prophylaxis (PrEP) in a national cohort of gay and bisexual men in the United States. JAIDS Journal of Acquired Immune Deficiency Syndromes, 74(3), 285-292. https://doi.org/10.1097/QAI.0000000000001251

Patel, R. R., Mena, L., Nunn, A., McBride, T., Harrison, L. C., Oldenburg, C. E., Liu, J., Mayer, K. H., \& Chan, P. A. (2017). Impact of insurance coverage on utilization of pre-exposure prophylaxis for HIV prevention. PLoS ONE, 12(5), 1-7. https://doi.org/10.1371/journal. pone.0178737

Pawson, M., \& Grov, C. (2018). 'It's just an excuse to slut around': gay and bisexual mens' constructions of HIV pre-exposure prophylaxis (PrEP) as a social problem. Sociology of Health \& Illness, 40(8), 1391-1403. https://doi.org/10.1111/1467-9566.12765

Pérez-Figueroa, R. E., Kapadia, F., Barton, S. C., Eddy, J. A., \& Halkitis, P. N. (2015). Acceptability of prep uptake among racially/ethnically diverse young men who have sex with men: The p18 study. AIDS Education and Prevention, 27(2), 112-125. https://doi. org/10.1521/aeap.2015.27.2.112

Persson, A. (2016). 'The world has changed': pharmaceutical citizenship and the reimagining of serodiscordant sexuality among couples with mixed HIV status in Australia. Sociology of Health \& Illness, 38(3), 380-395. https://doi.org/10.1111/1467-9566.12347

Philpot, S., Prestage, G., Holt, M., Haire, B., Maher, L., Hammoud, M., \& Bourne, A. (2020). Gay and bisexual men's perceptions of pre-exposure prophylaxis (PrEP) in a context of high accessibility: An Australian qualitative study. AIDS and Behavior. https://doi. org/10.1007/s10461-020-02796-3 
Pilkington, V., Hill, A., Hughes, S., Nwokolo, N., \& Pozniak, A. (2018). How safe is TDF/ FTC as PrEP? A systematic review and meta-analysis of the risk of adverse events in 13 randomised trials of PrEP. Journal of Virus Eradication, 4(4), 215-224. https://doi. org/10.1016/S2055-6640(20)30312-5

Pinto, R. M., Berringer, K. R., Melendez, R., \& Mmeje, O. (2018). Improving PrEP implementation through multilevel interventions: A synthesis of the literature. AIDS and Behavior, 22(11), 3681-3691. https://doi.org/10.1007/s10461-018-2184-4

Plomer, A., McCool-Myers, M., \& Apfelbacher, C. (2020). Perspectives on HIV PrEP care in Germany: qualitative insights from primary care physicians and specialists. AIDS Care, O(0), 1-7. https://doi.org/10.1080/09540121.2020.1778626

Poteat, T., Wirtz, A., Malik, M., Cooney, E., Cannon, C., Hardy, W. D., Arrington-Sanders, R., Lujan, M., \& Yamanis, T. (2019). A gap between willingness and uptake: Findings from mixed methods research on HIV prevention among Black and Latina transgender women. JAIDS Journal of Acquired Immune Deficiency Syndromes, 82(2), 131-140. https://doi. org/10.1097/QAI.0000000000002112

Poz.com. (2020). First generic Truvada now available in the United States. https://www.poz. com/article/first-generic-truvada-now-available-united-states

PrEPnu. (2017). Generic PrEP available in selected pharmacies for $€ 99,50$. https://www.prepnu.nl/en/2017/10/27/generic-prep-pharmacies/

Prestage, G., Down, I. A., Bradley, J., McCann, P. D., Brown, G., Jin, F., \& Hurley, M. (2012). Is optimism enough? Gay men囚s beliefs about HIV and their perspectives on risk and pleasure. Sexually Transmitted Diseases, 39(3), 167-172. https://doi.org/10.1097/OLQ. 0b013e31823e67a9

Prestage, G., Maher, L., Grulich, A., Bourne, A., Hammoud, M., Vaccher, S., Bavinton, B., Holt, M., \& Jin, F. (2019). Changes in behavior following PrEP initiation among Australian gay and bisexual men. Journal of Acquired Immune Deficiency Syndromes, 81(1), 1. https://doi.org/10.1097/qai.0000000000001976

Public Health England. (2019). HIV in the United Kingdom: Towards zero HIV transmissions by 2030 - 2019 report (Issue December 2018). https://www.gov.uk/government/publications/hiv-in-the-united-kingdom

Public Health Service Amsterdam. (2015). Study Protocol AMPrEP. https://www.trialregister. $\mathrm{nl} /$ trial/5302

Puppo, C., Spire, B., Morel, S., Génin, M., Béniguel, L., Costagliola, D., Ghosn, J., Mabire, X., Molina, J. M., Rojas Castro, D., \& Préau, M. (2020). How PrEP users constitute a community in the MSM population through their specific experience and management 
of stigmatization. The example of the French ANRS-PREVENIR study. AIDS Care, 0(0), 1-8. https://doi.org/10.1080/09540121.2020.1742863

Pyra, M. N., Haberer, J. E., Hasen, N., Reed, J., Mugo, N. R., \& Baeten, J. M. (2019). Global implementation of PrEP for HIV prevention: setting expectations for impact. Journal of the International AIDS Society, 22(8), 1-7. https://doi.org/10.1002/jia2.25370

Quinn, K. G., Christenson, E., Sawkin, M. T., Hacker, E., \& Walsh, J. L. (2019). The Unanticipated Benefits of PrEP for Young Black Gay, Bisexual, and Other Men Who Have Sex with Men. AIDS and Behavior, 0123456789. https://doi.org/10.1007/s10461-019-02747-7

Race, K. (2016). Reluctant objects: Sexual pleasure as a problem for HIV biomedical prevention. GLQ: A Journal of Lesbian and Gay Studies, 22(1), 1-31. https://doi. org/10.1215/10642684-3315217

Rana, J., Wilton, J., Fowler, S., Hart, T. A., Bayoumi, A. M., \& Tan, D. H. S. (2018). Trends in the awareness, acceptability, and usage of HIV pre-exposure prophylaxis among atrisk men who have sex with men in Toronto. Canadian Journal of Public Health, 109(3), 342-352. https://doi.org/10.17269/s41997-018-0064-3

Reisner, S. L., Moore, C. S., Asquith, A., Pardee, D. J., Sarvet, A., Mayer, G., \& Mayer, K. H. (2019). High risk and low uptake of pre-exposure prophylaxis to prevent HIV acquisition in a national online sample of transgender men who have sex with men in the United States. Journal of the International AIDS Society, 22(9). https://doi.org/10.1002/jia2.25391

Reitsema, M., Hoek, A. J. van, van der Loeff, M. S., Hoornenborg, E., van Sighem, A., Wallinga, J., van Benthem, B., \& Xiridou, M. (2020). Preexposure prophylaxis for men who have sex with men in the Netherlands. AIDS, 34(4), 621-630. https://doi.org/10.1097/ QAD.0000000000002469

Rendina, H. J., \& Parsons, J. T. (2018). Factors associated with perceived accuracy of the Undetectable $=$ Untransmittable slogan among men who have sex with men: Implications for messaging scale-up and implementation: Implications. Journal of the International AIDS Society, 21(1), 1-10. https://doi.org/10.1002/jia2.25055

Rendina, H. J., Whitfield, T. H. F., Grov, C., Starks, T. J., \& Parsons, J. T. (2017). Distinguishing hypothetical willingness from behavioral intentions to initiate HIV pre-exposure prophylaxis (PrEP): Findings from a large cohort of gay and bisexual men in the U.S. Social Science and Medicine, 172, 115-123. https://doi.org/10.1016/j.socscimed.2016.10.030

Ribeiro, S., \& Rocha, M. (2019). Pre-exposure prophylaxis counseling in a community sexual health clinic for men who have sex with men in Lisbon, Portugal. Acta Medica Portuguesa, 32(6), 441-447. https://doi.org/10.20344/amp.11474

Rice, W. S., Stringer, K. L., Sohail, M., Crockett, K. B., Atkins, G. C., Kudroff, K., Batey, D. 
S., Hicks, J., Turan, J. M., Mugavero, M. J., \& Turan, B. (2019). Accessing pre-exposure prophylaxis (PrEP): Perceptions of current and potential PrEP users in Birmingham, Alabama. AIDS and Behavior, 23(11), 2966-2979. https://doi.org/10.1007/s10461-01902591-9

Rivierez, I., Quatremere, G., Spire, B., Ghosn, J., \& Rojas Castro, D. (2018). Lessons learned from the experiences of informal PrEP users in France : results from the ANRS- PrEPage study. AIDS Care, O(0), 1-6. https://doi.org/10.1080/09540121.2018.1468014

Rodger, A. J., Cambiano, V., Phillips, A. N., Bruun, T., Raben, D., Lundgren, J., Vernazza, P., Collins, S., Degen, O., Corbelli, G. M., Estrada, V., Geretti, A. M., Beloukas, A., Beloukas, A., Coll, P., Antinori, A., Nwokolo, N., Rieger, A., Prins, J. M., ... Janeiro, N. (2019). Risk of HIV transmission through condomless sex in serodifferent gay couples with the HIV-positive partner taking suppressive antiretroviral therapy (PARTNER): final results of a multicentre, prospective, observational study. The Lancet, 393(10189), 2428-2438. https://doi.org/10.1016/S0140-6736(19)30418-0

Roehr, B. (2012). FDA approves first drug to prevent HIV infection. BMJ, 345, e4879-e4879. https://doi.org/10.1136/bmj.e4879

Rogers, E. M. (2010). Diffusion of innovations. Simon and Schuster.

Rojas Castro, D., Delabre, R. M., \& Molina, J. (2019). Give PrEP a chance: moving on from the "risk compensation" concept. Journal of the International AIDS Society, 22(S6). https:// doi.org/10.1002/jia2.25351

Rojas Castro, D., Delabre, R. M., Morel, S., Michels, D., \& Spire, B. (2019). Community engagement in the provision of culturally competent HIV and STI prevention services: lessons from the French experience in the era of PrEP. Journal of the International AIDS Society, 22(S6), 72-74. https://doi.org/10.1002/jia2.25350

Rolle, C.-P., Rosenberg, E. S., Siegler, A. J., Sanchez, T. H., Luisi, N., Weiss, K., Cutro, S., del Rio, C., Sullivan, P. S., \& Kelley, C. F. (2017). Challenges in translating PrEP interest into uptake in an observational study of young black MSM. JAIDS Journal of Acquired Immune Deficiency Syndromes, 76(3), 250-258. https://doi.org/10.1097/QAI.0000000000001497

Rosenthal, E., Piroth, L., Cua, E., Joulié, A., Ravaux, I., Chauveau, M., Lacombe, K., Cotte, L., Bonnard, P., Weiss, L., Longuet, M., Pradier, C., \& Cacoub, P. (2014). Preexposure prophylaxis (PrEP) of HIV infection in France: A nationwide cross-sectional study (PREVIC study). AIDS Care, 26(2), 176-185. https://doi.org/10.1080/09540121.2013.803014

Roux, P., Fressard, L., Suzan-Monti, M., Chas, J., Sagaon-Teyssier, L., Capitant, C., Meyer, L., Tremblay, C., Rojas-Castro, D., Pialoux, G., Molina, J.-M., \& Spire, B. (2018). Is on-demand HIV pre-exposure prophylaxis a suitable tool for men who have sex with men who 
practice chemsex? Results from a substudy of the ANRS-IPERGAY trial. JAIDS Journal of Acquired Immune Deficiency Syndromes, 79(2), e69-e75. https://doi.org/10.1097/ QAI.0000000000001781

Rozhnova, G., Heijne, J., Bezemer, D., van Sighem, A., Presanis, A., De Angelis, D., \& Kretzschmar, M. (2018). Elimination prospects of the Dutch HIV epidemic among men who have sex with men in the era of preexposure prophylaxis. AIDS, 32(17), 2615-2623. https://doi.org/10.1097/QAD.0000000000002050

Sagaon-Teyssier, L., Suzan-Monti, M., Demoulin, B., Capitant, C., Lorente, N., Préau, M., Mora, M., Castro, D. R., Chidiac, C., Chas, J., Meyer, L., Molina, J.-M., Spire, B., \& Group, for the A. I. S. (2016). Uptake of PrEP and condom and sexual risk behavior among MSM during the ANRS IPERGAY trial. AIDS Care, 28(sup1), 48-55. https://doi.org/10.1080/0 9540121.2016.1146653

Schnall, R., Travers, J., Rojas, M., \& Carballo-Diéguez, A. (2014). EHealth interventions for HIV prevention in high-risk men who have sex with men: A systematic review. Journal of Medical Internet Research, 16(5), 1-10. https://doi.org/10.2196/jmir.3393

Schneider, K., Gray, R. T., \& Wilson, D. P. (2014). A cost-effectiveness analysis of HIV preexposure prophylaxis for men who have sex with men in Australia. Clinical Infectious Diseases, 58(7), 1027-1034.

Schwartz, J., \& Grimm, J. (2017). PrEP on Twitter: Information, barriers, and stigma. Health Communication, 32(4), 509-516. https://doi.org/10.1080/10410236.2016.1140271

Schwartz, J., \& Grimm, J. (2019). Stigma communication surrounding PrEP: The experiences of a sample of men who have sex with men. Health Communication, 34(1), 84-90. https:// doi.org/10.1080/10410236.2017.1384430

Shover, C. L., Javanbakht, M., Shoptaw, S., Bolan, R. K., Lee, S. J., Parsons, J. T., Rendina, J., \& Gorbach, P. M. (2018). HIV preexposure prophylaxis initiation at a large community clinic: Differences between eligibility, awareness, and uptake. Am J Public Health, 108(10), 1408-1417. https://doi.org/10.2105/ajph.2018.304623

Singer, M., Bulled, N., Ostrach, B., \& Mendenhall, E. (2017). Syndemics and the biosocial conception of health. The Lancet, 389(10072), 941-950. https://doi.org/10.1016/S01406736(17)30003-X

Smit, P. J., Brady, M., Carter, M., Fernandes, R., Lamore, L., Meulbroek, M., Ohayon, M., Platteau, T., Rehberg, P., Rockstroh, J. K., \& Thompson, M. (2012). HIV-related stigma within communities of gay men: a literature review. AIDS Care, 24(4), 405-412. https://doi.org/ 10.1080/09540121.2011.613910

Smith, D. K., Herbst, J. H., Zhang, X., \& Rose, C. E. (2015). Condom effectiveness for HIV 
prevention by consistency of use among men who have sex with men in the United States. JAIDS Journal of Acquired Immune Deficiency Syndromes, 68(3), 337-344. https://doi. org/10.1097/QAI.0000000000000461

Smith, D. K., Sullivan, P. S., Cadwell, B., Waller, L. A., Siddiqi, A., Mera-Giler, R., Hu, X., Hoover, K. W., Harris, N. S., \& McCallister, S. (2020). Evidence of an association of increases in pre-exposure prophylaxis coverage with decreases in human immunodeficiency virus diagnosis rates in the United States, 2012-2016. Clinical Infectious Diseases, Xx Xxxx. https://doi.org/10.1093/cid/ciz1229

Smith, D. K., Van Handel, M., Wolitski, R. J., Stryker, J. E., Hall, H. I., Prejean, J., Koenig, L. J., \& Valleroy, L. A. (2015). Vital signs: estimated percentages and numbers of adults with indications for preexposure prophylaxis to prevent HIV acquisition-United States, 2015. Morbidity and Mortality Weekly Report, 64(46), 1291-1295.

Snowden, J. M., Chen, Y. H., McFarland, W., \& Raymond, H. F. (2017). Prevalence and characteristics of users of pre-exposure prophylaxis (PrEP) among men who have sex with men, San Francisco, 2014 in a cross-sectional survey: Implications for disparities. Sexually Transmitted Infections, 93(1), 52-55. https://doi.org/10.1136/sextrans-2015-052382

Spieldenner, A. (2016). PrEP whores and HIV prevention: The queer communication of HIV pre-exposure prophylaxis (PrEP). Journal of Homosexuality, 63(12), 1685-1697. https:// doi.org/10.1080/00918369.2016.1158012

Spinelli, M. A., Laborde, N., Kinley, P., Whitacre, R., Scott, H. M., Walker, N., Liu, A. Y., Gandhi, M., \& Buchbinder, S. P. (2020). Missed opportunities to prevent HIV infections among pre-exposure prophylaxis users: a population-based mixed methods study, San Francisco, United States. Journal of the International AIDS Society, 23(4), 1-6. https://doi. org/10.1002/jia2.25472

Staritsky, L. E., Van Aar, F., Visser, M., Op de Coul, E. L. M., Heijne, J., Götz, H. M., Nielen, M., van Sighem, A. I., \& Van Benthem, B. H. B. (2020). Sexually transmitted infections in the Netherlands in 2019. In RIVM report 2020-0052. https://doi.org/10.21945/RIVM2020-0052

Starks, T. J., Rendina, H. J., Breslow, A. S., Parsons, J. T., \& Golub, S. A. (2013). The psychological cost of anticipating HIV stigma for HIV-negative gay and bisexual men. AIDS and Behavior, 17(8), 2732-2741. https://doi.org/10.1007/s10461-013-0425-0

Stolte, I. G., Dukers, N. H. T. M., Geskus, R. B., Coutinho, R. A., \& De Wit, J. B. F. (2004). Homosexual men change to risky sex when perceiving less threat of HIV/AIDS since availability of highly active antiretroviral therapy. AIDS, 18(2), 303-309. https://doi. org/10.1097/00002030-200401230-00021 
Storholm, E. D., Volk, J. E., Marcus, J. L., Silverberg, M. J., \& Satre, D. D. (2017). Risk perception, sexual behaviors, and PrEP adherence among substance-using men who have sex with men: A qualitative study. Prevention Science, 18(6), 737-747. https://doi.org/10.1007/ s11121-017-0799-8

Stutterheim, S. E., Bos, A., \& Schaalma, H. (2008). HIV-related stigma in the Netherlands. http://hivnet.org/downloads/pdf/reportplusproject.pdf

Stutterheim, S. E., Pryor, J. B., Bos, A. E. R., Hoogendijk, R., Muris, P., \& Schaalma, H. P. (2009). HIV-related stigma and psychological distress: the harmful effects of specific stigma manifestations in various social settings. AIDS, 23(17), 2353-2357. https://doi. org/10.1097/QAD.0b013e3283320dce

Stutterheim, S. E., Van Dijk, M., \& Jonas, K. J. (2020). The worldwide burden of HIV in transgender individuals: An updated systematic review and meta-analysis. Submitted for Publication.

Tellalian, D., Maznavi, K., Bredeek, U. F., \& Hardy, W. D. (2013). Pre-exposure prophylaxis (PrEP) for HIV infection: Results of a survey of HIV healthcare providers evaluating their knowledge, attitudes, and prescribing practices. AIDS Patient Care and STDs, 27(10), 553-559. https://doi.org/10.1089/apc.2013.0173

Tester, G., \& Hoxmeier, J. C. (2020). PrEP, TasP and the casual sex scripts among serodiscordant gay men. Journal of HIV/AIDS and Social Services, 0(0), 1-16. https://doi.org/10.108 0/15381501.2020.1753620

The Lancet. (2017). Syndemics: health in context. The Lancet, 389(10072), 881. https://doi. org/10.1016/S0140-6736(17)30640-2

Thomann, M., Grosso, A., Zapata, R., \& Chiasson, M. A. (2017). “WTF is PrEP?”: attitudes towards pre-exposure prophylaxis among men who have sex with men and transgender women in New York City. Culture, Health \& Sexuality, 1-15. https://doi.org/10.1080/136 91058.2017.1380230

Thorne, S. (2016). Interpretive Description. Routledge. https://doi.org/10.4324/9781315545196

Traeger, M. W., Schroeder, S. E., Wright, E. J., Hellard, M. E., Cornelisse, V. J., Doyle, J. S., \& Stoové, M. A. (2018). Effects of pre-exposure prophylaxis for the prevention of human immunodeficiency virus infection on sexual risk behavior in men who have sex with men: A systematic review and meta-analysis. Clinical Infectious Diseases, 67(5), 676-686. https://doi.org/10.1093/cid/ciy182

Tsai, A. C., Mendenhall, E., Trostle, J. A., \& Kawachi, I. (2017). Co-occurring epidemics, syndemics, and population health. The Lancet, 389(10072), 978-982. https://doi.org/10.1016/ S0140-6736(17)30403-8 
U.S. Food and Drug Administration. (2019). FDA approves second drug to prevent HIV infection as part of ongoing efforts to end the HIV epidemic. https://www.fda.gov/news-events/ press-announcements/fda-approves-second-drug-prevent-hiv-infection-part-ongoingefforts-end-hiv-epidemic

UNAIDS. (2016). Maximizing the potential of a new HIV prevention method: PrEP. https:// www.unaids.org/en/resources/presscentre/featurestories/2016/november/20161101_ PrEP

UNAIDS. (2019). UNAIDS Data 2019. https://www.unaids.org/en/resources/documents/2019/2019-UNAIDS-data

Underhill, K., Guthrie, K. M., Colleran, C., Calabrese, S. K., Operario, D., \& Mayer, K. H. (2018). Temporal fluctuations in behavior, perceived HIV risk, and willingness to use pre-exposure prophylaxis (PrEP). Archives of Sexual Behavior, 47(7), 2109-2121. https:// doi.org/10.1007/s10508-017-1100-8

Vaccher, S. J., Gianacas, C., Templeton, D. J., Poynten, I. M., Haire, B. G., Ooi, C., Foster, R., McNulty, A., Grulich, A. E., \& Zablotska, I. B. (2017). Baseline preferences for daily, event-driven, or periodic hiv pre-exposure prophylaxis among gay and bisexual men in the prelude demonstration project. Frontiers in Public Health, 5(December), 1-8. https:// doi.org/10.3389/fpubh.2017.00341

Van Bergen, J. (2019). De rol van de huisarts bij PrEP revisited. https://www.soaaids.nl/nl/ seksoa-magazine/rol-van-huisarts-bij-prep-revisited

Van Dijk, M., de Wit, J. B. F., Guadamuz, T., Martinez, J. E., \& Jonas, K. J. (2020). Slow uptake of PrEP: Behavioral predictors and the influence of price on PrEP uptake among MSM with a high interest in PrEP. Manuscript under Revision.

Van Sighem, A., Gras, L., Smit, C., Stolte, I., \& Reiss, P. (2019). HIV Monitoring Report 2019. www.hiv-monitoring.nl

Visser, M., Van Aar, F., Van Oeffelen, A. A. M., Op de Coul, E. L. M., Hofstraat, S. H. I., Heijne, J., Den Daas, C., Hoenderboom, B. M., Van Wees, D. A., Basten, M., Woestenberg, P. J., Götz, H. M., van Sighem, A. I., De Hoon, S., \& Van Benthem, B. H. B. (2017). Sexually transmitted infections including HIV, in the Netherlands in 2016. https://www.rivm.nl/ publicaties/sexually-transmitted-infections-including-hiv-in-netherlands-in-2016

Volk, J. E., Hojilla, J. C., Hurley, L., Silverberg, M. J., Skarbinski, J., Satre, D. D., Hare, C. B., Alexeeff, S., \& Marcus, J. L. (2020). PrEP continuum of care and new HIV infections: Long-term follow-up in a large clinical cohort. 23rd International AIDS Conference, abstract OAC0807.

Vuylsteke, B., Reyniers, T., Lucet, C., Nöstlinger, C., Deblonde, J., Libois, A., Sauvage, A. S., 
Deprez, E., Goffard, J. C., Allard, S. D., Florence, E., Demeester, R., Callens, S., \& Laga, M. (2019). High uptake of pre-exposure prophylaxis (PrEP) during early roll-out in Belgium: Results from surveillance reports. Sexual Health, 16(1), 80-83. https://doi.org/10.1071/ SH18071

Wainberg, M. A., Hull, M. W., Girard, P. M., \& Montaner, J. S. G. (2016). Achieving the 90-9090 target: incentives for HIV testing. The Lancet Infectious Diseases, 16(11), 1215-1216. https://doi.org/10.1016/S1473-3099(16)30383-8

Wang, L., Moqueet, N., Lambert, G., Grace, D., Rodrigues, R., Cox, J., Lachowsky, N. J., Noor, S. W., Armstrong, H. L., Tan, D. H. S., Burchell, A. N., Ma, H., Apelian, H., Knight, J., Messier-Peet, M., Jollimore, J., Baral, S., Hart, T. A., Moore, D. M., \& Mishra, S. (2020). Population-level sexual mixing according to HIV status and preexposure prophylaxis use among men who have sex with men in Montreal, Canada: Implications for HIV prevention. American Journal of Epidemiology, 189(1), 44-54. https://doi.org/10.1093/aje/ kwz231

Wang, X., Nwokolo, N., Korologou-Linden, R., Hill, A., Whitlock, G., Day-Weber, I., McClure, M. O., \& Boffito, M. (2018). InterPrEP: internet-based pre-exposure prophylaxis with generic tenofovir disoproxil fumarate/emtrictabine in London - analysis of pharmacokinetics, safety and outcomes. HIV Medicine, 19(1), 1-6. https://doi.org/10.1111/ hiv. 12528

Weiss, K., Bratcher, A., Sullivan, P., \& Siegler, A. (2018). Geographic access to PrEP clinics among US MSM: Documenting PrEP deserts. Conference on Retroviruses and Opportunistic Infections (CROI). https://doi.org/10.2196/publichealth.7902.5.

Whitfield, T. H. F., John, S. A., Rendina, H. J., Grov, C., \& Parsons, J. T. (2018). Why I quit pre-exposure prophylaxis (PrEP)? A mixed-method study exploring reasons for PrEP discontinuation and potential re-initiation among gay and bisexual men. AIDS and Behavior, 22(11), 3566-3575. https://doi.org/10.1007/s10461-018-2045-1

Whitfield, T. H. F., Jones, S. S., Wachman, M., Grov, C., Parsons, J. T., \& Rendina, H. J. (2019). The impact of pre-exposure prophylaxis (PrEP) use on sexual anxiety, satisfaction, and esteem among gay and bisexual men. Journal of Sex Research, 56(9), 1128-1135. https:// doi.org/10.1080/00224499.2019.1572064

World Health Organization. (2014). Consolidated guidelines on HIV prevention, diagnosis, treatment and care for key populations. WHO Guidelines, July, 184. http://apps.who.int/ iris/bitstream/10665/128048/1/9789241507431_eng.pdf?ua=1

World Health Organization. (2015). Guideline on when to start antiretroviral therapy and on pre-exposure prophylaxis for HIV. September, 1-76. 
World Health Organization. (2020). Latest HIV estimates and updates on HIV policies uptake, July 2020. In Global HIV, Hepatitis and STI Programmes World Health Organization (Issue July). https://www.who.int/hiv/data/2019_global_summary_web_v2.pdf

Yang, D., Chariyalertsak, C., Wongthanee, A., Kawichai, S., Yotruean, K., Saokhieo, P., Guadamuz, T., Suwanvanichkij, V., Beyrer, C., \& Chariyalertsak, S. (2013). Acceptability of pre-exposure prophylaxis among men who have sex with men and transgender women in Northern Thailand. PLoS ONE, 8(10), e76650. https://doi.org/10.1371/journal. pone. 0076650

Young, I., \& McDaid, L. (2014). How acceptable are antiretrovirals for the prevention of sexually transmitted HIV?: A review of research on the acceptability of oral pre-exposure prophylaxis and treatment as prevention. AIDS and Behavior, 18(2), 195-216. https://doi. org/10.1007/s10461-013-0560-7

Zablotska, I. B., Grulich, A. E., Phanuphak, N., Anand, T., Janyam, S., Poonkasetwattana, M., Baggaley, R., van Griensven, F., \& Lo, Y.-R. (2016). PrEP implementation in the Asia-Pacific region: opportunities, implementation and barriers. Journal of the International AIDS Society, 19(Suppl 6), 21119. https://doi.org/10.7448/IAS.19.7.21119

Zablotska, I. B., Prestage, G., de Wit, J., Grulich, A. E., Mao, L., \& Holt, M. (2013). The informal use of antiretrovirals for preexposure prophylaxis of HIV infection among gay men in Australia. Journal of Acquired Immune Deficiency Syndromes, 62(3), 334-338. https:// doi.org/10.1097/QAI.0b013e31827e854a

Zablotska, I. B., Selvey, C., Guy, R., Price, K., Holden, J., Schmidt, H.-M., McNulty, A., Smith, D., Jin, F., Amin, J., Cooper, D. A., \& Grulich, A. E. (2018). Expanded HIV pre-exposure prophylaxis (PrEP) implementation in communities in New South Wales, Australia (EPIC-NSW): design of an open label, single arm implementation trial. BMC Public Health, 18(1), 210. https://doi.org/10.1186/s12889-017-5018-9

Zablotska, I. B., Vaccher, S. J., Bloch, M., Carr, A., Foster, R., Grulich, A., Guy, R., McNulty, A., Ooi, C., Pell, C., Poynten, I. M., Prestage, G., Ryder, N., Templeton, D., Cheung, C., Gianacas, C., Holt, M., Kaldor, J., Mackie, B., ... Wright, E. (2019). High adherence to HIV pre-exposure prophylaxis and no HIV seroconversions despite high levels of risk behaviour and STIs: The Australian demonstration study PrELUDE. AIDS and Behavior, 23(7), 1780-1789. https://doi.org/10.1007/s10461-018-2290-3

Zardiashvili, T. (2019). Pre-exposure prophylaxis (PrEP) in Eastern Europe and Central Asia: First lessons to learn. https://ecom.ngo/en/library/prep_report_cases/ 
REFERENCES 
SUMMARY 
Pre-exposure prophylaxis (PrEP) is a novel biomedical intervention that is highly effective in reducing HIV infections. The World Health Organization (WHO) recommends the use of PrEP for people at high risk of HIV, such as men who have sex with men (MSM). However, PrEP uptake has been slow, because of limited accessibility and affordability. In the Netherlands, PrEP became available for $€ 500$ per 30 pills in 2016, which was unaffordable for most people who are at risk of HIV. Consequently, some MSM obtained PrEP via informal channels such as (online) pharmacies and clinics abroad, and through pill sharing of people living with HIV who take antiretroviral therapy. Because informal PrEP users are usually not medically supervised, concerns were raised about misinformation and incorrect use of PrEP. This thesis aims to assess whether informal PrEP use occurs in the Netherlands, and what risks are related to informal PrEP use. Therefore, we investigated the interest in $\operatorname{PrEP}$ and the experiences of PrEP users in the years prior to formal PrEP implementation in the Netherlands.

We examined the attitudes towards PrEP and interest in PrEP in the study reported in chapter 2. We conducted this study in the summer of 2016, before PrEP was available in the Netherlands. At the time, many MSM were already aware of PrEP and had correct knowledge of PrEP. About half of the participants were interested in PrEP and had the intention to start using PrEP when it would become available. We found that MSM who may benefit from PrEP (i.e. those with high-risk behavior) were indeed those who were more interested in PrEP. In addition, we found that the recruitment strategy was also significantly related to interest in PrEP: Participants of the research panel AmsterdamPinkPanel were less interested in PrEP compared to participants recruited via convenience sampling. This indicates that it is important to take recruitment strategies and sample characteristics into account in explaining study findings.

In the study reported in chapter 3 we qualitatively explored the ways in which PrEP users obtained PrEP and what their experiences were regarding accessing PrEP. Participants who first bought PrEP informally abroad or online, had mostly switched to buying PrEP formally at the pharmacy in the Netherlands as soon as PrEP became more affordable in 2018. Some participants indicated that they had to switch general practitioners (GP) because their previous GP had moralistic ideas about sex and PrEP and therefore did not want to prescribe PrEP. Overall, informal PrEP users were quite capable of arranging PrEP and the care associated with PrEP for themselves, although renal function testing was not always done regularly, as some PrEP users did not find this necessary. In addition to accessing PrEP, we also explored community responses and sexual behavior of PrEP users. Most PrEP users received positive 
responses to their PrEP usage. However, stigmatizing reactions also emerged. For example, PrEP users were rejected for sex because other MSM thought that PrEP users would have more sexually transmitted infections (STIs) due to irresponsible sexual behavior. PrEP users described that their condom use had generally decreased since they started using PrEP. However, they still used condoms in certain situations, depending on the perceived risk and their familiarity with the sex partner. An important benefit of PrEP use was a reduced fear of HIV. PrEP users felt more in control of their sex life and experienced more sexual freedom, which made them more willing to experiment with sex practices they did not dare before.

In the study reported in chapter 4 we examined correlates, and potential barriers, of PrEP uptake. We found that PrEP uptake was higher after the price drop of PrEP in 2018, among MSM who were in a favorable financial situation, and among MSM who had previously had a post-exposure prophylaxis (PEP) treatment. Our study showed that the price of PrEP mainly played a role in the PrEP uptake among MSM in an average financial situation. MSM with a low financial status still could not afford to pay PrEP despite the price drop, while MSM with a high financial status could afford PrEP regardless of the price.

In the study reported in chapter 5 we quantitatively investigated the psychosocial benefits of PrEP. PrEP users reported consistent improvements in quality of sex life after PrEP initiation. Reduced fear for HIV was related to improved quality of sex life. In contrast to our expectations, this association was not mediated by condom use. Drug use in a sexual context (chemsex) was high in this group, but PrEP users reported no increase in experimentation with novel drugs since PrEP initiation.

In the study reported in chapter 6 we investigated in more detail the attitudes of PrEP users when having sex with men living with HIV. The majority of PrEP users indicated to have sex with men living with HIV. They reported high levels of reduced fear for HIV and increased comfort when having sex with men living with HIV. They also indicated that since they started using PrEP, they felt safer to have condomless sex with men living with HIV. These feelings increased over time and showed that PrEP users may have been more confident relying on PrEP over time. The findings suggest that PrEP can play a role in overcoming the serodivide, and that this manifests quite soon at the onset of PrEP use.

In the general discussion in chapter 7 the main findings are summarized, contextualized and critically appraised. The implications of the findings are also discussed, with a focus on settings in the Netherlands, in other countries with a similar PrEP situation, as well as more 
broadly from an international perspective. A strength of this thesis is that it reports on the first research program in the Netherlands to study PrEP use outside a standard healthcare setting, such as in the demonstration trial AMPrEP. Informal PrEP use exists and may continue to exist, as MSM may find it more convenient or more affordable. The studies in this thesis show that this informal use of PrEP can work quite well, but it may mainly reach MSM who have a high community engagement, and who are consequently well informed about PrEP. For them, the transition to formal PrEP likely proceeds smoothly as soon as formal PrEP is accessible and affordable. There is a gap between interest in PrEP and actual uptake of PrEP among MSM in the Netherlands. PrEP mainly reaches MSM who can afford it, even though there is high interest from other MSM with high-risk behaviors. PrEP should be seen as part of a range of HIV prevention strategies that can be combined. The combination of PrEP and Treatment as Prevention (TasP) probably has the most impact in reducing the incidence of new HIV infections. The studies in this thesis demonstrate that the benefits of PrEP go beyond just HIV prevention: PrEP has a positive impact on the quality of sex life by reducing fear for HIV. PrEP uptake may increase if the psychosocial benefits of PrEP are promoted. Future research should focus on people who stop using PrEP, as they remain at risk for HIV and condoms are not always a viable strategy for them. Research is also needed on PrEP use among other high-risk groups, such as young MSM, migrants, and transgender individuals. 


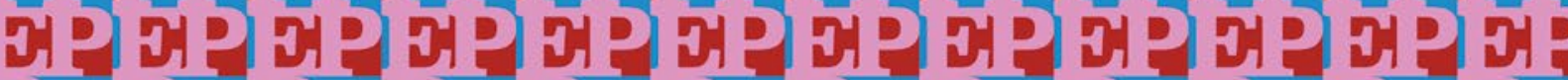

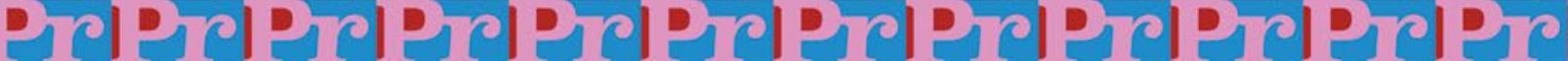
胡

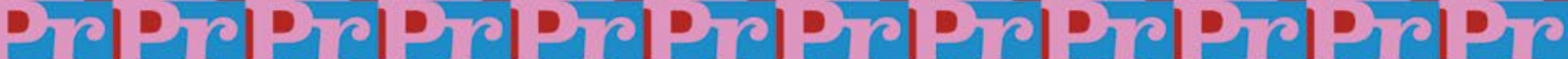

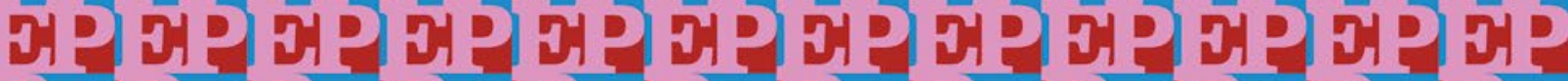

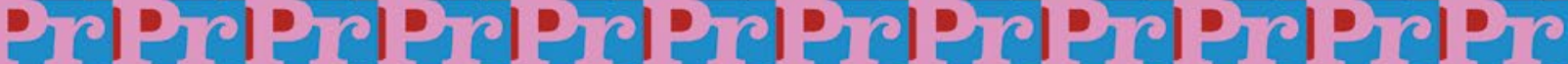
斯 D. 频

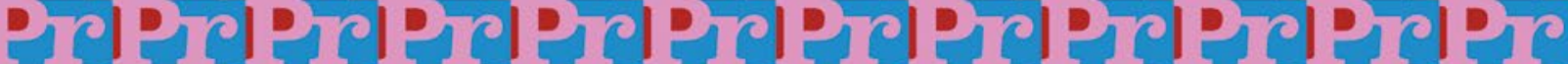

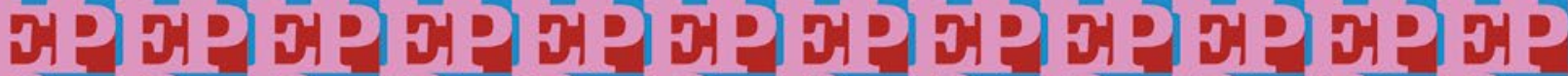

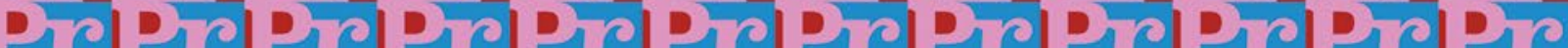

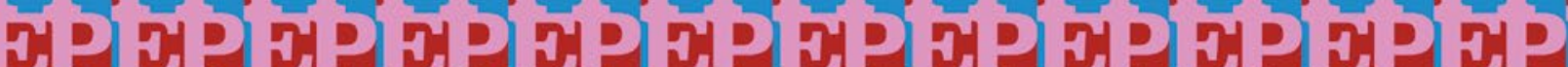
ת

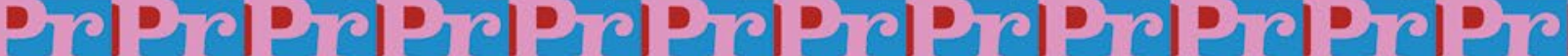
斩

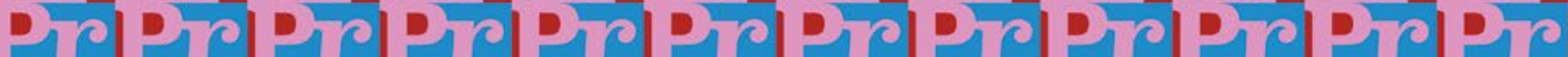
故

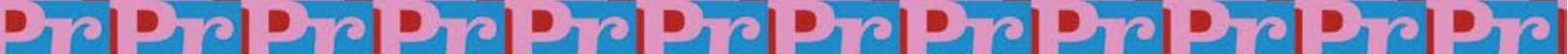
秋

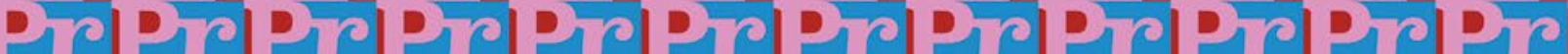
故

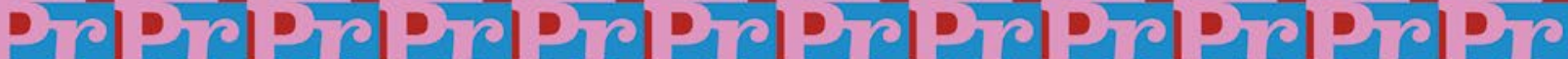
的

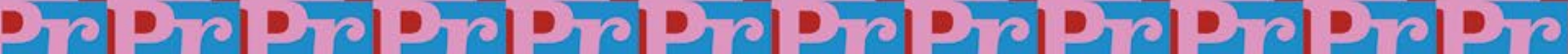
胡

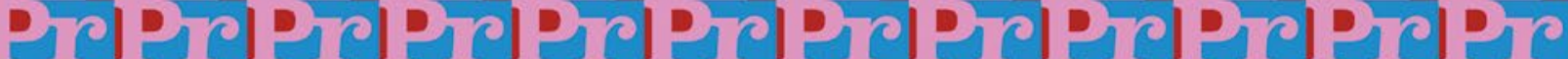

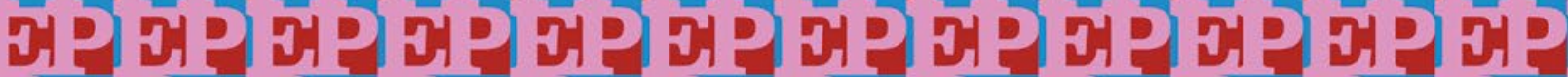

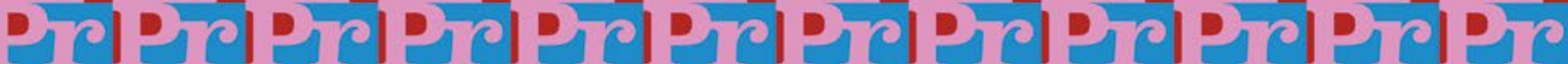

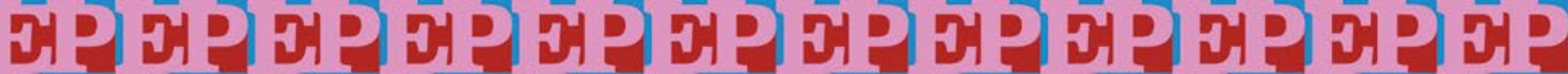

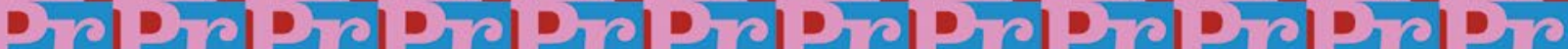

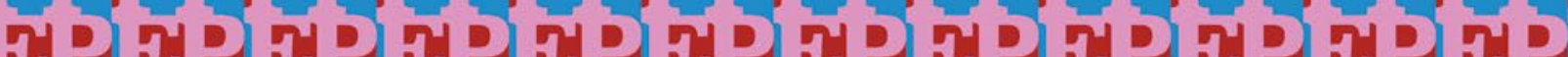
ת

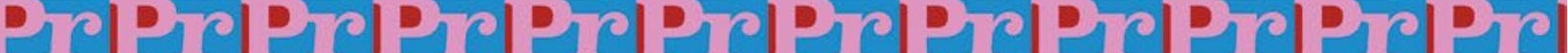
斯 
SAMENVATTING 
Pre-exposure prophylaxis (PrEP) is een nieuwe biomedische interventie die zeer effectief is in het voorkomen van hiv-infecties. De Wereldgezondheidsorganisatie (WHO) beveelt het gebruik van PrEP aan voor mensen met een verhoogd risico op hiv, zoals mannen die seks hebben met mannen (MSM). De ingebruikname van PrEP verloopt echter traag, vanwege de beperkte toegankelijkheid en betaalbaarheid. In Nederland kwam PrEP in 2016 beschikbaar voor $€ 500$ per 30 pillen, wat onbetaalbaar was voor de meeste mensen met een verhoogd risico op hiv. Daarom hebben sommige MSM PrEP verkregen via informele kanalen zoals (online) apotheken en klinieken in het buitenland, en door het delen van pillen door mensen met hiv die antiretrovirale medicatie gebruiken. Omdat informele PrEP-gebruikers meestal niet medisch begeleid worden, zijn er zorgen geuit over onjuiste informatie en onjuist gebruik van PrEP. Dit proefschrift beoogt te onderzoeken of informeel PrEP-gebruik in Nederland voorkomt en welke risico's verbonden zijn aan informeel PrEP-gebruik. Daarom hebben we de interesse in PrEP en de ervaringen van PrEP-gebruikers in de jaren voorafgaand aan de formele invoering van PrEP in Nederland onderzocht.

We onderzochten de attitudes ten opzichte van PrEP en de interesse in PrEP in het onderzoek dat in hoofdstuk 2 wordt beschreven. We hebben dit onderzoek uitgevoerd in de zomer van 2016, voordat PrEP in Nederland beschikbaar was. Op dat moment waren veel MSM al op de hoogte van PrEP en hadden ze correcte kennis over PrEP. Ongeveer de helft van de deelnemers was geïnteresseerd in PrEP en had de intentie om PrEP te gaan gebruiken wanneer het beschikbaar zou komen. We vonden dat MSM die mogelijk baat hebben bij PrEP (zoals degenen met hoog risicogedrag) inderdaad degenen waren die meer geïnteresseerd waren in PrEP. Daarnaast vonden we dat de wervingsstrategie voor de steekproeftrekking ook significant gerelateerd was aan de interesse in PrEP: Deelnemers van het onderzoekspanel AmsterdamPinkPanel waren minder geïnteresseerd in PrEP in vergelijking met deelnemers die werden geworven via gemakssteekproeven ('convenience sampling'). Dit geeft aan dat het belangrijk is om bij de interpretatie van onderzoeksresultaten rekening te houden met de wervingsstrategieën en de steekproefkenmerken.

In het onderzoek dat in hoofdstuk 3 wordt beschreven, hebben we op kwalitatieve wijze onderzocht hoe PrEP-gebruikers PrEP bemachtigen en wat hun ervaringen zijn met de toegang tot PrEP. Deelnemers die PrEP voor het eerst informeel in het buitenland of online kochten, waren meestal overgestapt op het formeel aanschaffen van PrEP bij de apotheek in Nederland zodra PrEP in 2018 betaalbaarder werd. Sommige deelnemers gaven aan dat ze moesten wisselen van huisarts omdat hun vorige huisarts moralistische ideeën had over seks en PrEP en 
daarom PrEP niet wilde voorschrijven. Over het algemeen waren informele PrEP-gebruikers goed in staat om PrEP en de zorg die bij PrEP hoort voor zichzelf te regelen, hoewel nierfunctietests niet altijd regelmatig werden uitgevoerd, omdat sommige PrEP-gebruikers dit niet nodig vonden. Naast de toegang tot PrEP hebben we ook de reacties van de community en het seksueel gedrag van PrEP-gebruikers onderzocht. De meeste PrEP-gebruikers ontvingen positieve reacties op hun PrEP-gebruik. Er kwamen echter ook stigmatiserende reacties naar voren. Zo werden PrEP-gebruikers afgewezen voor seks omdat andere MSM dachten dat PrEP-gebruikers meer seksueel overdraagbare aandoeningen (soa's) zouden hebben als gevolg van onverantwoordelijk seksueel gedrag. PrEP-gebruikers beschreven dat hun condoomgebruik over het algemeen was afgenomen sinds ze PrEP gebruikten. Ze gebruikten nog steeds condooms in bepaalde situaties, afhankelijk van het veronderstelde risico en hun vertrouwdheid met de sekspartner. Een belangrijk voordeel van PrEP-gebruik was een verminderde angst voor hiv. PrEP-gebruikers voelden zich meer in controle over hun seksleven en ervaarden meer seksuele vrijheid, waardoor ze meer geneigd waren te experimenteren met seksuele activiteiten die ze voorheen niet durfden.

In het onderzoek dat in hoofdstuk 4 wordt beschreven, hebben we gekeken naar correlaties, en potentiële barrières, van PrEP-gebruik. We vonden dat PrEP-gebruik hoger was na de prijsdaling van PrEP in 2018, bij MSM die zich in een gunstige financiële situatie bevonden, en bij MSM die eerder een post-exposure profylaxe (PEP) behandeling hadden gehad. Ons onderzoek toonde aan dat de prijs van PrEP vooral een rol speelde bij PrEP-gebruik bij MSM in een gemiddelde financiële situatie. MSM met een lage financiële status konden zich ondanks de prijsdaling nog steeds geen PrEP veroorloven, terwijl MSM met een hoge financiële status zich wel PrEP konden veroorloven, ongeacht de prijs.

In het onderzoek dat in hoofdstuk 5 wordt beschreven, hebben we de psychosociale voordelen van PrEP kwantitatief onderzocht. PrEP-gebruikers rapporteerden consistente verbeteringen in de kwaliteit van het seksleven sinds zij PrEP gebruikten. Verminderde angst voor hiv was gerelateerd aan een verbeterde kwaliteit van het seksleven. In tegenstelling tot onze verwachtingen werd deze associatie niet gemedieerd door condoomgebruik. Drugsgebruik in een seksuele context (chemsex) was hoog in deze groep, maar PrEP-gebruikers rapporteerden geen toename in experimenteren met nieuwe drugs sinds zij PrEP gebruikten.

In het onderzoek dat in hoofdstuk 6 wordt beschreven, hebben we de attitudes van PrEP-gebruikers bij het hebben van seks met mannen die leven met hiv onderzocht. De meerder- 
heid van de PrEP-gebruikers gaf aan seks te hebben met mannen die leven met hiv. Zij rapporteerden een hoge mate van verminderde angst voor hiv en een verhoogd comfort bij het hebben van seks met mannen die leven met hiv. Ze gaven ook aan dat ze zich sinds ze PrEP gebruikten veiliger voelden om condoomloze seks te hebben met mannen die leven met hiv. Deze gevoelens namen in de loop van de tijd toe en lieten zien dat gebruikers van PrEP in de loop van de tijd misschien meer vertrouwen kregen in PrEP. De bevindingen suggereren dat PrEP een rol kan spelen bij het overkomen van de 'serodivide', en dat dit zich vrij snel manifesteert bij het begin van het gebruik van PrEP.

In de algemene discussie in hoofdstuk 7 worden de belangrijkste bevindingen samengevat, gecontextualiseerd en kritisch beoordeeld. De implicaties van de bevindingen worden ook besproken, met aandacht voor situaties in Nederland, in andere landen met een vergelijkbare PrEP-situatie, maar ook meer in het algemeen vanuit een internationaal perspectief. Een sterk punt van dit proefschrift is dat het verslag doet van de eerste onderzoeken in Nederland naar informeel PrEP-gebruik buiten de reguliere gezondheidszorg om, zoals in de demonstratieproef AMPrEP. Informeel PrEP-gebruik bestaat en blijft mogelijk bestaan, omdat MSM het handiger of betaalbaarder vinden. De onderzoeken in dit proefschrift laten zien dat dit informele gebruik van PrEP redelijk goed gaat, maar het bereikt vooral MSM die een hoge betrokkenheid bij de community hebben en die daardoor goed op de hoogte zijn van PrEP. Voor hen verloopt de overgang naar formeel PrEP waarschijnlijk soepel zodra formeel PrEP toegankelijk en betaalbaar is. Er is een discrepantie tussen de interesse in PrEP en het daadwerkelijke gebruik van PrEP bij MSM in Nederland. PrEP bereikt vooral MSM die het zich kunnen veroorloven, ook al is er grote belangstelling van andere MSM met risicovol gedrag. PrEP moet worden gezien als onderdeel van een reeks van hiv-preventiestrategieën die kunnen worden gecombineerd. De combinatie van PrEP en Treatment as Prevention (TasP) heeft waarschijnlijk het meeste effect op het verminderen van nieuwe hiv-infecties. De onderzoeken in dit proefschrift tonen aan dat de voordelen van PrEP verder gaan dan alleen hiv-preventie: PrEP heeft een positief effect op de kwaliteit van het seksleven door het verminderen van angst voor hiv. Het gebruik van PrEP kan toenemen als de psychosociale voordelen van PrEP worden benadrukt. Toekomstig onderzoek kan zich richten op mensen die stoppen met het gebruik van PrEP, aangezien zij risico blijven lopen op hiv en condooms voor hen niet altijd een haalbare preventiestrategie zijn. Er is ook onderzoek nodig naar het gebruik van PrEP onder andere risicogroepen, zoals jonge MSM, migranten en transgender personen. 
SAMENVATTING 


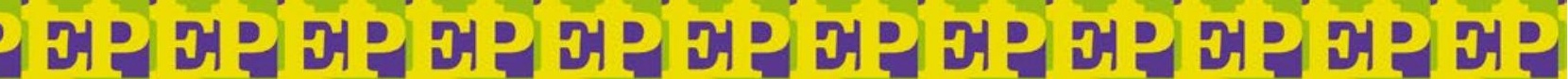

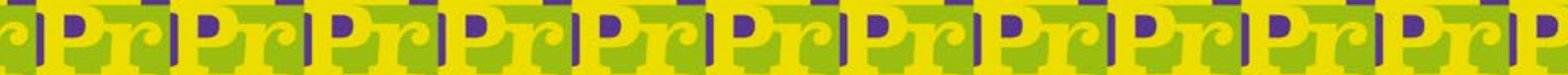

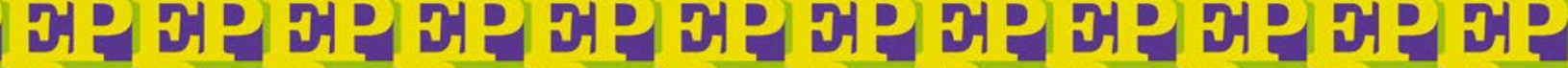

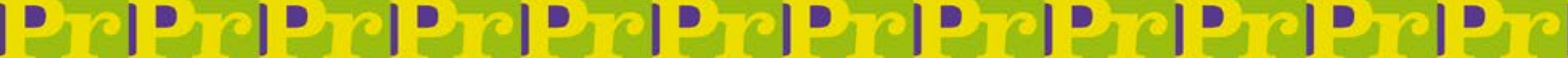

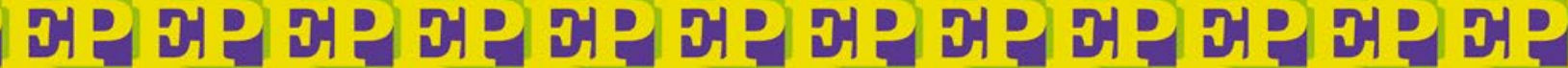
|D

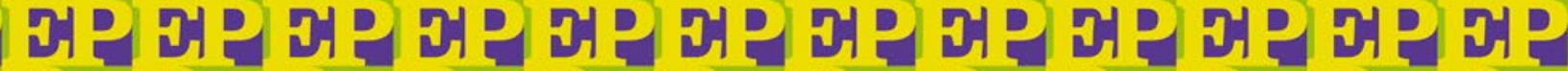

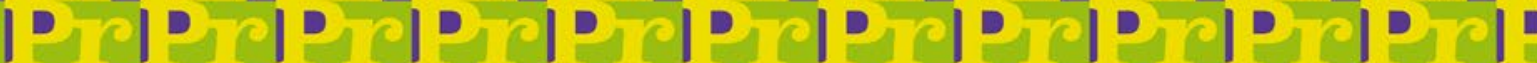

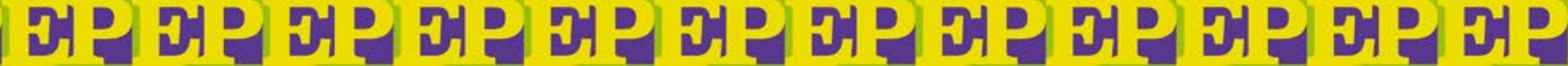

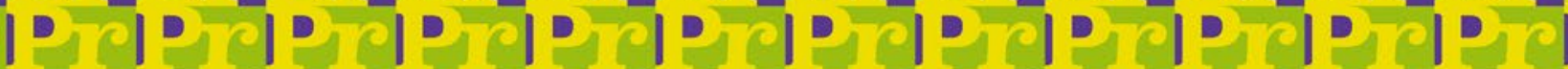

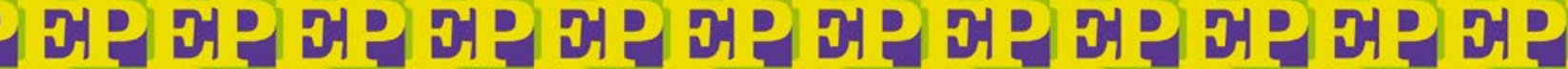

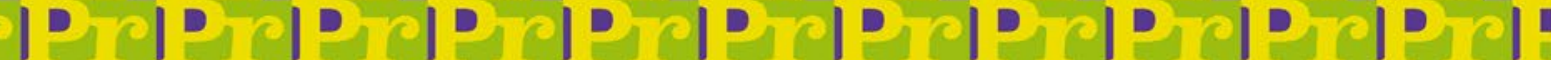

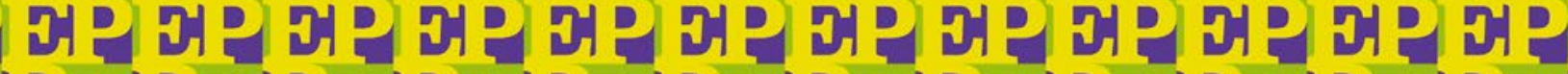

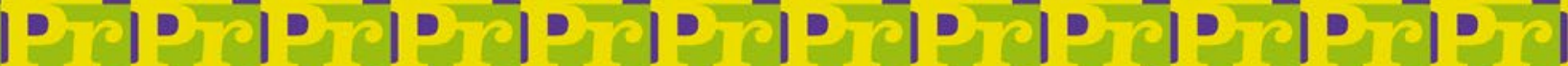

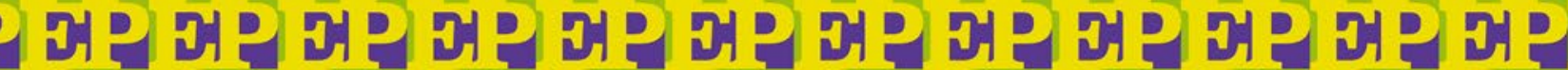

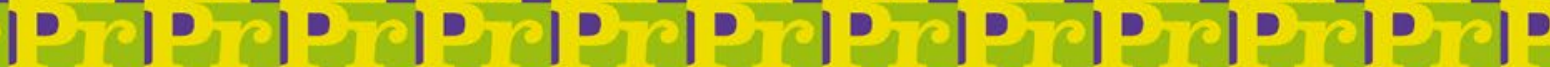

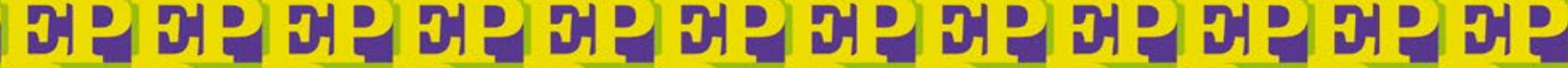

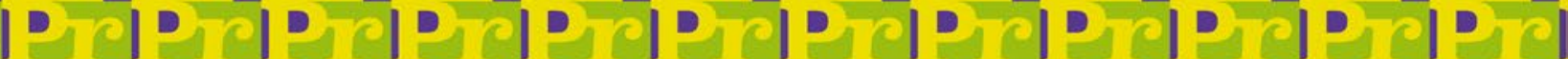

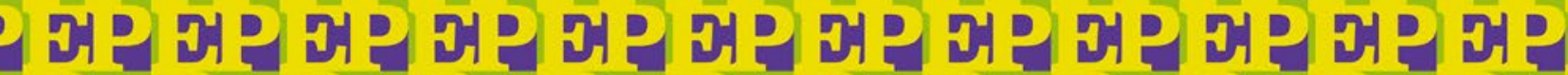

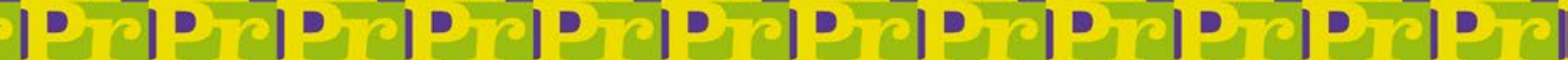

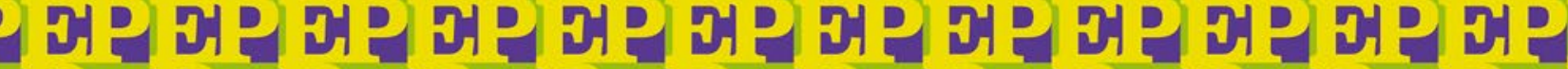

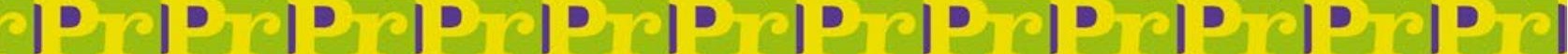

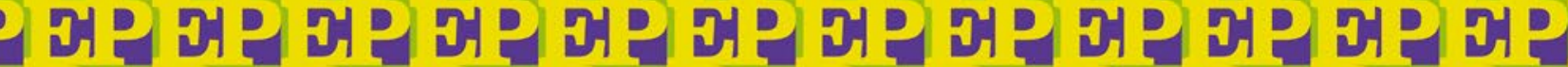

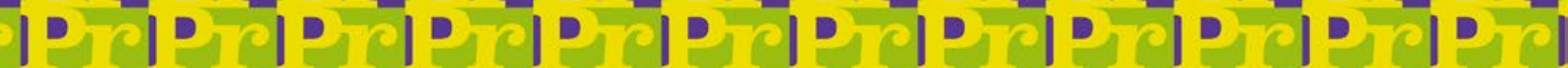

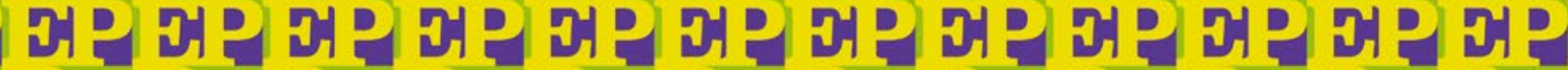

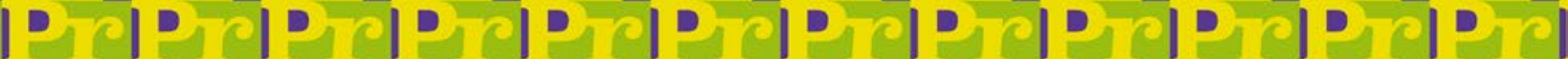

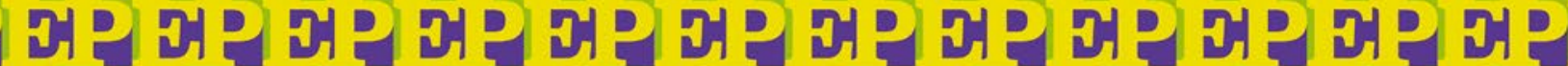

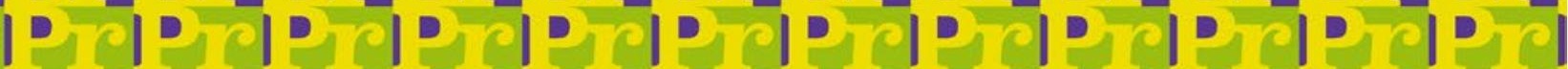

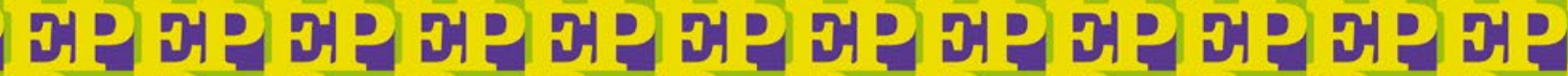

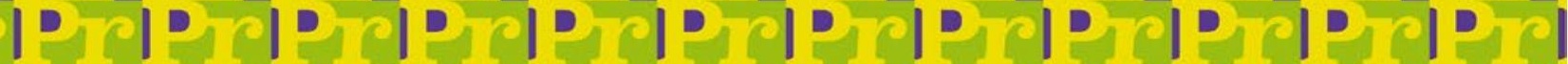

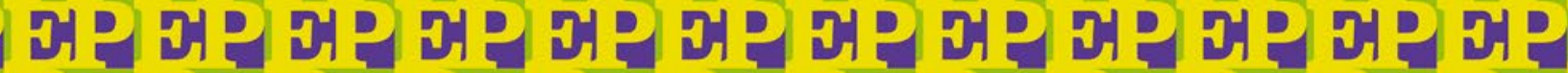

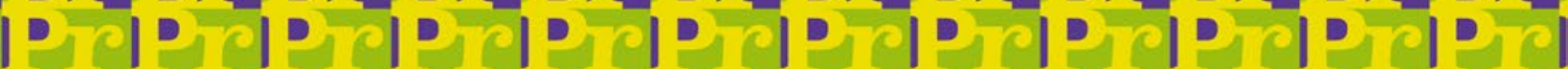

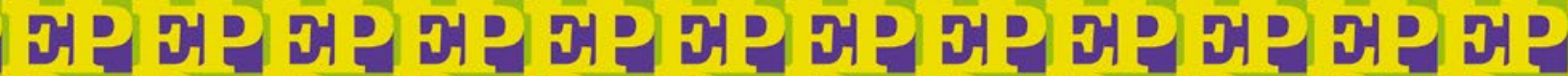


IMPACT PARAGRAPH 
Men who have sex with men (MSM) are at increased risk of HIV. Pre-exposure prophylaxis (PrEP) is an established form of biomedical prevention strategy that greatly reduces the risk of HIV transmission. The main goal of this dissertation was to investigate the attitudes of MSM towards PrEP and the experiences of PrEP users during the early years of the introduction of PrEP in the Netherlands.

The studies in this thesis are among the first studies in the Netherlands on the informal use of PrEP outside a standard healthcare setting. We focused on MSM who obtain PrEP through informal channels, such as in clinics and pharmacies abroad. Previous concerns about incorrect PrEP use (and subsequent potential HIV resistance) seem unjustified, as most informal PrEP users in our studies used PrEP correctly and engaged in regular testing. Most informal PrEP users have made a smooth transition to formal PrEP use as soon as PrEP became more affordable in the Netherlands in 2018. Now that the price of PrEP has dropped, and PrEP has also become available through the public health centers, PrEP will be accessible to a broader audience. It is important that these MSM are well informed about PrEP, which is why the information on the website mantotman.nl has been extended with information about PrEP. This information was created in the project We Are PrEPared in collaboration with GGD Amsterdam, GGD Gelderland-Zuid and SoaAids Nederland. The findings of the studies in this thesis on PrEP interest and PrEP knowledge among MSM provided insights for the needs assessment and the development of this information.

In the Netherlands, PrEP services have been implemented in the public health centers in a project with room for 6,500 people. However, it can still be possible that MSM go to the general practitioner for PrEP care, for example when there is no place in the local GGD, or when it is more convenient for them (e.g. due to travel distance). It is therefore important that healthcare professionals outside the public health centers, such as general practitioners, are also well informed about PrEP. This PrEP-related healthcare should also be accessible to informal PrEP users. The PrEP users in our research indicated that general practitioners were often not well informed about PrEP, or even refused to prescribe PrEP, highlighting a need for improving PrEP knowledge among general practitioners. Improving the knowledge of healthcare professionals was also part of the goals of the working group We Are PrEPared.

The findings of our studies are also relevant for PrEP use in other countries. In most countries in Europe and Central Asia PrEP is not yet available or formally implemented, so MSM who want to use PrEP are dependent on informal PrEP use. Nevertheless, informal PrEP use 
is not widely discussed in the scientific literature. In the studies in this thesis we found that informal PrEP users are highly self-reliant. This does not imply that formal implementation of PrEP should be discontinued. The formal implementation of PrEP is important, because it makes PrEP accessible to more people. Our research showed that the price of PrEP is a significant barrier to PrEP uptake, and this barrier can be removed when PrEP is included in the national healthcare system and reimbursed by health insurance. This will ensure that people in vulnerable financial situations also have access to PrEP. In addition, when implementing PrEP, the accessibility of PrEP for other vulnerable groups such as migrants and transgender people should also be taken into account, by increasing PrEP awareness among these groups.

The studies in this thesis fit into the scientific debate on PrEP in which there is an increasing demand for sociobehavioral studies on PrEP. Initially, research focused on the effectiveness and safety of PrEP. However, PrEP use has implications that go beyond just HIV prevention. In the studies in this thesis we found that PrEP has psychosocial benefits in addition to its preventive effect. PrEP users reported an increase in quality of sex life, and this was mainly related to a decrease in fear of HIV. Decreased fear of HIV and increased comfort were also reported in the context of having sex with men living with HIV. This shows that PrEP can play a role in reducing HIV stigma: PrEP users are more open to having sex with men living with HIV. HIV community organizations could promote and emphasize the role of PrEP in stigma reduction campaigns.

PrEP should be seen as part of a range of HIV prevention strategies that can be combined. Promoting the psychosocial benefits in addition to its preventive effect may help to increase PrEP uptake and to reach the full potential of PrEP in preventing new HIV infections. Combining multiple HIV prevention strategies is essential to reach the Aidsfonds goal of zero new HIV infections in the Netherlands. 
DANKWOORD 
In mijn tijd in Maastricht heb ik veel nieuwe mensen ontmoet, die me inspireerden, waar ik van geleerd heb, en die me tot steun zijn geweest. Op deze plek wil ik graag mijn dank aan hen uitspreken.

Allereerst wil ik mijn promotoren, Kai en John, bedanken. Ik ben jullie beiden erg dankbaar dat jullie vertrouwen in mij hadden en mij de kans hebben gegeven om dit promotieonderzoek uit te voeren. Kai, ik vond het fijn dat je me vanaf het begin bij allerlei projecten betrok, zoals het Flash PrEP project waarbij we voor meetings naar Parijs en Brussel reisden. Hierdoor raakte ik snel vertrouwd met de onderzoeksonderwerpen en het werkveld. Je stimuleerde me om ambitieus te zijn en maakte het mogelijk dat ik een paar maanden onderzoek in Hong Kong kon doen. Dat is een waardevolle ervaring voor mij geweest. John, je hielp me om mijn werk met wat afstand te bekijken, waardoor ik nieuwe inzichten kreeg in de data. Je motiveerde me om niet al te zwaarmoedig met commentaar van reviewers om te gaan en je hielp me om mijn Engelse geschreven taal te verbeteren. Dank voor jullie begeleiding.

Graag wil ik ook Rob bedanken. Je was in het begin betrokken als promotor in dit project. Je stimuleerde me om mijn onderzoek ook vanuit een breder perspectief van gezondheidspsychologie te bekijken. Ook ben ik je dankbaar dat je me leerde kennismaken met de wereld van Intervention Mapping. I would like to express my gratitude to Thomas and Joel, who have been actively involved in this project from the start. Thomas, I am very thankful that you invited me to provide a workshop in Bangkok. I learned a lot from this experience, and it was a pleasure to visit your university. Joel, I appreciate your critical comments on our papers. Your perspective and insights were very valuable to improve the papers. Rebecca, thank you for your helpful contribution to the qualitative research.

Ik wil graag een aantal partijen bedanken die essentieel geweest zijn voor de uitvoering van dit onderzoeksproject. Ik ben dankbaar dat we dit project konden uitvoeren dankzij financiering van het Aidsfonds. Ik wil het team van PrEPnu bedanken voor hun hulp bij het werven van deelnemers voor dit onderzoek. I would like to thank Dr. Deyn of the Silom Pulse Clinic in Bangkok for helping us recruiting participants for our research. Ik wil ook alle deelnemers, en in het bijzonder de deelnemers die we mochten interviewen, bedanken voor hun tijd en interesse in het onderzoek. Hun antwoorden zijn onmisbaar geweest om meer inzicht te krijgen in informeel PrEP ge- 
bruik in Nederland. Ik wil de leden van de community advisory board (Piet, Richard, Cor, Leon, Alfie, Sjoerd) bedanken voor hun input die richting gaf aan dit onderzoek.

Uiteraard wil ik ook al mijn collega's van Work and Social Psychology bedanken. Ik waardeer de gezellige lunches die we met elkaar hadden in de keuken. Verder wil ik nog een paar collega's in het bijzonder bedanken:

Bram en Stefan, mijn paranimfen. Ik ben blij dat jullie mijn kamergenoten van het eerste uur waren en dat we vrienden zijn geworden. Sommigen hadden ons graag als boyband gezien, maar helaas ontbreekt ons het muzikaal talent (uitgezonderd Stefan, je speelt heel goed piano). Bram, bedankt dat je altijd een luisterend oor bood, en dat je me hebt laten kennismaken met Beb Vuyk en exotische fruitsoorten. Stefan, bedankt voor je steun en voor je aanmoedigingen in the gym. Mochten we alle drie ooit voor een alternatief carrièrepad willen kiezen, dan hoop ik met jullie een horecagelegenheid in Randwyck te openen, zodat onze collega's geen droge broodjes meer hoeven te eten tijdens de lunch.

Alicia, bedankt dat je me het nachtleven van Maastricht hebt laten zien. Wat hebben we leuke avonden in de Sway gehad, en ook gezellige etentjes in Barcelona en Maastricht. Rosine, bedankt voor de gouden tip die mijn schrijftempo enorm verhoogd heeft en natuurlijk ook voor je tips bij het spelen van Stardew Valley! Ook vind ik het leuk dat je, samen met Darta, me (een beetje) hebt leren klimmen. Marie, I liked that we went through the same phases of our $\mathrm{PhD}$ projects at the same time, so we had a lot of support from each other. Vera, ik vind het leuk dat je in het laatste jaar mijn kamergenoot was en bedankt voor je fijne samenwerking in het IPS-project. Bona, thank you for the good conversations and for showing your musical talent at the department meetings. Karlijn, we waren elkaars lievelingscollega's bij het begeleiden van studenten. Ik heb ook goede herinneringen aan het samen vieren van onze verjaardagen, met oranjekoek en echte taart (géén vlaai). Gerda, bedankt voor de fijne gesprekken en goede adviezen, en dat ik mocht meedenken bij je huizenjacht. Sarah, bedankt voor de samenwerking in het meta-analyse project. Ola, Lizette, Tamika, and Haoyi, thank you for the interesting discussions during our hiv lab meetings. Karen, bedankt voor je tips en begeleiding bij statistische analyses. Louk, bedankt voor de leuke gesprekken. Fleurie en Margje, bedankt voor de begeleiding in het BKO-programma. Gemma, bedankt voor je samenwerking en begeleiding in het IPS-project. 
Fred, hoewel je ons vaak hebt aangeboden om je schoenen te poetsen, ben ik blij dat je toch ook andere taken voor me in de aanbieding had, tige tank. Gerjo, bedankt dat je de leescommissie wilde voorzitten en dat je me betrok bij het schrijven van reviews. Mariëlla en Trudy, bedankt voor al jullie ondersteuning bij administratieve taken.

Ik wil ook graag mijn vrienden bedanken die me gesteund hebben in de afgelopen jaren. Geert en Rolf, bij jullie kon ik altijd terecht, of ik nou last had van een writer's block of van een lawaaiige buurvrouw. Ik ben jullie dankbaar dat jullie me motiveerden tijdens moeilijke perioden. Joris, Kjell, Tamme en Doede, bedankt voor jullie steun en alle gezellige momenten tijdens logeerpartijtjes, vakanties en feestjes. Christien en Arjen, wat fijn dat we al zo lang vrienden zijn en nog steeds regelmatig contact hebben. Maria, bedankt voor de fijne gesprekken, je steun en je goede adviezen. Prachilla, bedankt voor al je bezoekjes aan Maastricht en je enthousiasme en steun. Nikki, Julia en Antoine, bedankt voor jullie samenwerking bij Wereld Aids Dag Limburg. Darío, thank you for designing the cover and illustrations for this thesis.

Tenslotte wil ik mijn familie bedanken. Mijn opa en oma, met trots en belangstelling volgden jullie mijn opleiding en werk. Mijn ouders, jullie staan altijd voor me klaar en ik ben dankbaar voor alle kansen die jullie me hebben gegeven om me te ontwikkelen. Jullie zorgden voor een warme thuisbasis van waaruit ik mijn eigen leven vrij kon vormgeven. Kasper en Liza, Julius en Fien, ik vind het fijn dat we zo goed met elkaar op kunnen schieten en het is altijd gezellig als we met elkaar samen zijn. 


\section{Curriculum vitae}

Mart van Dijk was born in Sneek, the Netherlands on March 23, 1990. He attended secondary education at RSG Magister Alvinus from which he graduated in 2008. He studied Psychology at the University of Groningen and participated in the honours programme of the Bachelor Psychology. After receiving his Bachelor's degree in 2011, he continued studying at the same university and he received his research master's degree in Behavioural and Cognitive Neurosciences in 2013. During and after his master, he worked as a research assistant at the VU University in Amsterdam. In 2015-2016, he worked as an IT specialist in cryptography at ING Bank. In 2016, he started his PhD project 'PrEP among MSM in the Netherlands: attitudes, intentions, modes of acquisition and sexual risk taking' at Maastricht University that resulted in this dissertation. Besides his work, he volunteers at the charity dinner AmsterdamDiner and organises activities for World Aids Day Limburg. 